\title{
Estruturas Polissimpléticas e Multissimpléticas em Variedades e Fibrados
}

\author{
Leandro Gustavo Gomes
}

TESE APRESENTADA

$\mathrm{AO}$

INSTITUTO DE MATEMÁTICA E ESTATÍSTICA

DA

UNIVERSIDADE DE SÃO PAULO

PARA

OBTENÇÃO DO GRAU DE DOUTOR

EM

CIÊNCIAS

\author{
Área de Concentração: Matemática Aplicada \\ Orientador : Prof. Dr. Frank Michael Forger
}

Durante a elaboração deste trabalho o autor recebeu apoio do CNPq e da CAPES

- São Paulo, fevereiro de 2007 - 



\section{Estruturas Polissimpléticas e Multissimpléticas em Variedades e Fibrados}

Este exemplar corresponde à redação final da tese devidamente corrigida e defendida

por Leandro Gustavo Gomes e aprovada pela comissão julgadora.

São Paulo, 28 de fevereiro de 2007.

\section{COMISSÃO JULGADORA}

- Prof. Dr. Frank Michael Forger (orientador) - IME-USP

- Prof. Dr. Paolo Piccione - IME-USP

- Prof. Dr. Plamen Emilov Kochloukov - UNICAMP

- Prof. Dr. Caio José Colletti Negreiros - UNICAMP

- Prof. Dr. Henrique Bursztyn - IMPA 



\title{
Resumo
}

Neste trabalho, introduzimos uma nova classe de formas multilineares alternadas e de formas diferenciais, chamadas de formas polilagrangeanas (no caso de formas a valores vetoriais) ou multilagrangeanas (no caso de formas parcialmente horizontais em relação a um subespaço ou subfibrado dado), que são caracterizadas pela existência de um tipo especial de subespaço ou subfibrado maximal isotrópico chamado, respectivamente, de polilagrangeano ou multilagrangeano. Revela-se que estas constituem o arcabouço adequado para a formulação de um teorema de Darboux em nível algébrico. Combinando esta nova estrutura algébrica com propriedades padrão de integrabilidade $(d \omega=0)$ nos permite deduzir o teorema de Darboux no contexto geométrico (existência de coordenadas locais canônicas). Estruturas polissimpléticas e multissimpléticas, inclusive todas aquelas que aparecem no formalismo hamiltoniano covariante da teoria clássica dos campos, são contidas como caso especial.

\begin{abstract}
In this thesis, we introduce a new class of multilinear alternating forms and of differential forms called polylagrangean (in the case of vector-valued forms) or multilagrangean (in the csae of forms that are partially horizontal with respect to a given subspace or subbundle), characterized by the existence of a special type of maximal isotropic subspace or subbundle called polylagrangean or multilagrangean, respectively. As it turns out, these constitute the adequate framework for the formulation of an algebraic Darboux theorem. Combining this new algebraic structure with standard integrability conditions $(d \omega=0)$ allows us to derive a geometric Darboux theorem (existence of canonical local coordinates). Polysymplectic and multisymplectic structures, including all those that appear in the covariant hamiltonian formalism of classical field theory, are contained as a special case.
\end{abstract}





\section{Agradecimentos}

"Taught me my alphabet to say -

To lisp my very earliest word

While in the wild wood I did lie,

A child - with a most knowing eye."

(Trecho do poema "Romance", de E.A. Poe.)

À minha família, minha referência e casa. Seu patriarca, Levy, e matriarca, Rosângela, são os maiores responsáveis por tudo o que consegui até hoje.

Ao Prof. Michael Forger, pela orientação e companheirismo nesta jornada. Levarei muito destes momentos nos quais empreendemos um duro trabalho.

A algumas pessoas com quem eu compartilhei muitas idéias, sentimentos e momentos, fossem eles tristes ou felizes, simples ou grandes. Embora não as cite aqui, nunca as esqueço.

A quem me apresentou Edgar Allan Poe. 



\section{Conteúdo}

Introdução $\quad$ i

1 Formas polilagrangeanas e multilagrangeanas: teoria algébrica $\quad 1$

1.1 Formas a valores vetoriais em espaços vetoriais . . . . . . . . . . . . 5

1.2 Formas parcialmente horizontais em espaços vetoriais . . . . . . . . . . 13

1.3 O símbolo . . . . . . . . . . . . . . . . . . 17

1.4 Exemplos canônicos . . . . . . . . . . . . . . . . . 18

1.4.1 A forma polilagrangeana canônica . . . . . . . . . . . . . . . . 19

1.4.2 A forma multilagrangeana canônica . . . . . . . . . . . . . . . 20

1.5 Espaços vetoriais polilagrangeanos . . . . . . . . . . . . . . . 22

1.6 Espaços vetoriais multilagrangeanos . . . . . . . . . . . . . . . . 24

2 Formas polilagrangeanas e multilagrangeanas: teoria diferencial $\quad 27$

2.1 Cálculo de Cartan para formas verticais . . . . . . . . . . . . . . 28

2.2 Variedades polilagrangeanas . . . . . . . . . . . . . . . . 31

2.3 Fibrados poli- e multilagrangeanos . . . . . . . . . . . . . . . 33

2.4 O símbolo . . . . . . . . . . . . . . . . . . . 35

2.5 O teorema de Darboux . . . . . . . . . . . . . . . . . . 39 
3 Estruturas poli- e multissimpléticas $\quad 43$

3.1 Espaços vetoriais polissimpléticos . . . . . . . . . . . . . . 44

3.2 Espaços vetoriais multissimpléticos . . . . . . . . . . . . . . . 53

3.3 Variedades e fibrados poli- e multissimpléticos . . . . . . . . . . . . 57

A Lema de Poincaré 


\section{Introdução}

A geometria multissimplética é cada vez mais reconhecida como provedora do arcabouço matemático adequado para a teoria clássica dos campos do ponto de vista hamiltoniano, assim como a geometria simplética para a mecânica clássica. Infelizmente o desenvolvimento desta nova área da geometria diferencial tem sido obstruído, até o momento, pela inexistência de uma definição plenamente satisfatória do conceito de uma estrutura multissimplética que, idealmente, deveria ser matematicamente simples tanto quanto em harmonia com as necessidades das aplicações à física.

A presente tese tem como objetivo apresentar uma nova proposta para superar este problema.

Inicialmente consideremos uma simples analogia. As formas simpléticas encontradas na mecânica clássica podem ser localmente escritas na forma

$$
\omega=d q^{i} \wedge d p_{i},
$$

onde $q^{1}, \ldots, q^{N}, p_{1}, \ldots, p_{N}$ são um tipo especial de coordenadas locais no espaço de fase, denominadas coordenadas canônicas ou coordenadas de Darboux. Introduzindo o tempo $t$ e a energia $E$ como variáveis adicionais (o que é essencial, por exemplo, para incorporar sistemas não autônomos no formalismo simplético da mecânica hamiltoniana), esta equação é substituida por

$$
\omega=d q^{i} \wedge d p_{i}+d E \wedge d t,
$$

onde $t, q^{1}, \ldots, q^{N}, p_{1}, \ldots, p_{N}, E$ podem ser vistas como coordenadas canônicas em um espaço de fase estendido. De modo semelhante, as formas multissimpléticas que aparecem na teoria clássica dos campos sobre uma variedade $n$-dimensional $M$, esta representando o espaçotempo, podem ser localmente escritas na forma

$$
\omega=d q^{i} \wedge d p_{i}^{\mu} \wedge d^{n} x_{\mu}-d p \wedge d^{n} x,
$$


onde $x^{\mu}, q^{i}, p_{i}^{\mu}, p(1 \leqslant \mu \leqslant n, 1 \leqslant i \leqslant N)$ podem, mais uma vez, ser consideradas como coordenadas canônicas em algum espaço de multifase estendido. Aqui, as $x^{\mu}$ são coordenadas (locais) para $M$ enquanto que $p$ é uma única variável energia (a menos de um sinal), $d^{n} x$ é a forma de volume (local) induzida pelas $x^{\mu}$ e $d^{n} x_{\mu}$ é a $(n-1)$-forma (local) obtida pela contração de $d^{n} x \operatorname{com} \partial_{\mu} \equiv \partial / \partial x^{\mu}$ :

$$
d^{n} x_{\mu}=\mathrm{i}_{\partial_{\mu}} d^{n} x
$$

A idéia de introduzir "variáveis multimomento" rotuladas por um índice espaço-temporal adicional (de modo que há $n$ variáveis multimomento $p_{i}^{\mu}$ para cada variável posição $q^{i}$ ) retrocede aos trabalhos de De Donder [5] e Weyl [20] nos anos 1930 (e talvez ainda mais) e tem sido reconhecido desde então como sendo um ingrediente essencial e inevitável para qualquer tentativa de chegar a uma formulação hamiltoniana totalmente covariante da teoria clássica dos campos. Contudo o problema de determinar qual é o ambiente geométrico adequado para este tipo de estrutura tem desafiado tanto matemáticos quanto físicos há décadas, como testemunhado pelo grande número de tentativas que podem ser encontradas na literatura, sendo quase todas falhas, em algum ponto ou outro, ou porque não levaram em conta uma ou várias das sutilezas envolvidas no problema ou porque optaram pela "saída fácil" de artificialmente invocar estruturas adicionais. Um exemplo típico de uma tal estrutura adicional é a escolha de uma conexão como "campo de fundo", o que exclui a possibilidade de incorporar teorias de calibre, nas quais conexões são variáveis dinâmicas: obviamente um formalismo que se propõe como geral mas deixa de acomodar teorias de calibre dificilmente será muito útil.

Uma exceção notável é provida pela construção do espaço de multifase como fibrado dos cojatos em modelos da teoria clássica dos campos de primeira ordem cuja formulação hamiltoniana pode ser obtida de uma formulação lagrangeana pela transformação de Legendre. A partir de um fibrado $E$ sobre o espaço-tempo $M$, cujas seções são interpretadas como os campos básicos do modelo sob consideração, forma-se seu fibrado de jatos de primeira ordem $J E$, que é um fibrado sobre $M$ (com respeito à projeção fonte) cujas seções representam as possíveis primeiras derivadas dos campos básicos e que configura a arena natural para o formalismo lagrangeano. No próximo passo, usa-se o fato de que $J E$ também é um fibrado afim sobre $E$ (com respeito à projeção alvo) para formar seu dual afim $P=J^{\circledast} E$ que acaba sendo a arena natural para o formalismo hamiltoniano. Em particular, esse vem munido de uma forma multissimplética $\omega$ globalmente definida cuja representação em coordenadas canônicas tem a forma dada pela equação (3) acima. Notavelmente, neste caso, a forma multissimplética $\omega$ é (a menos de um sinal) a derivada exterior de uma forma multicanônica $\theta$ globalmente definida cuja representação em coordenadas canônicas é dada por

$$
\theta=p_{i}^{\mu} d q^{i} \wedge d^{n} x_{\mu}+p d^{n} x
$$

Detalhes desta construção podem ser encontrados na literatura $[4,6,8,9]$, sendo que ela providencia a única classe de exemplos de estruturas multissimpléticas conhecidas até o 
momento. No entanto, vale notar que ela é estritamente análoga à construção do espaço de fase na mecânica clássica como fibrado cotangente de um espaço de configuração, o que claramente indica que não deve ser a construção mais geral possível. O que está faltando é o análogo multissimplético de outros exemplos de variedades simpléticas que não podem ser realizadas como fibrados vetoriais e onde $\omega$ deixa de ser exata, tais como variedades de Kähler e órbitas coadjuntas.

Como primeira tentativa para abordar o problema, considere a opção óbvia de estender o conceito de uma estrutura simplética para o de uma estrutura multissimplética definindo uma variedade multissimplética como sendo uma variedade $P$ munida de uma forma diferencial $\omega$ de grau $n+1$ que é fechada $(d \omega=0)$ assim como não degenerada, i.e., satisfaz

$$
\mathrm{i}_{w} \omega(p)=0 \Longrightarrow w=0 \quad \text { para } p \in P, w \in T_{p} P \text {. }
$$

Obviamente esta definição capta parte dos aspectos relevantes mas é demasiadamente ampla para ser de uso prático. Em particular, ela é insuficiente para garantir a validade de um teorema de Darboux (exceto quando $n=1$ ).

O principal problema com a definição acima é que ela ignora por completo o papel do espaço-tempo. Mais precisamente, ela ignora o fato da variedade $P$ não ser apenas uma simples variedade mas sim o espaço total de um fibrado sobre uma variedade base $M$, a qual não apenas determina o grau da forma em questão ( $\operatorname{dim} M=n \Rightarrow \operatorname{deg} \omega=n+1)$ mas também provê a noção de formas horizontais (as 1-formas $d x^{\mu}$ e seus produtos exteriores), o que, grosso modo, nos permite remover um fator $(n-1)$-horizontal de $\omega$ e assim reduzir $\omega$ a uma coleção de 2-formas, conforme

$$
\omega=\omega^{(\mu)} \wedge d^{n} x_{\mu}
$$

onde

$$
\omega^{(\mu)}=d q^{i} \wedge d p_{i}^{\mu}-d p \wedge d x^{\mu}
$$

pois

$$
d x^{\mu} \wedge d^{n} x_{\nu}=\delta_{\nu}^{\mu} d^{n} x
$$

É claro que tal decomposição depende das coordenadas adotadas, mas a sua existência é um ingrediente importante que não deve ser negligenciado e sim deve ser reformulado de uma maneira que independe de coordenadas.

Em essência isto significa que não existe uma noção natural de variedade multissimplética, mas apenas a de um fibrado multissimplético cuja variedade base deve ser interpretada como o espaço-tempo sobre a qual campos clássicos são definidos. ${ }^{1}$ Para nossos propósitos aqui,

\footnotetext{
${ }^{1} \mathrm{Na}$ verdade, o conceito matemático mais geral de um campo, na teoria clássica, é que campos são seções de um fibrado sobre o espaço-tempo.
} 
a variedade base não precisa carregar nenhuma estrutura adicional, tal como uma métrica lorentziana ou, no âmbito euclideano, uma métrica riemanniana. Isso pode parecer estranho quando comparado com a situação na mecânica, onde estamos acostumados a usar variedades simpléticas e não fibrados simpléticos, mas isso é realmente uma particularidade da mecânica não relativística de sistemas autônomos, que se perde quando passamos para sistemas não autônomos ou sistemas relativísticos:

- Mesmo no contexto da mecânica clássica não relativística o tratamento de um sistema não autônomo requer uma extensão do espaço de fase convencional da teoria, que é uma variedade simplética $P_{0}$, digamos, adicionando uma cópia da reta real $\mathbb{R}$ como "eixo temporal" e, no intuito de evitar ter que trabalhar com variedades de contato tal como o "espaço de fase simplesmente estendido" $\mathbb{R} \times P_{0}$, uma outra cópia da mesma como "eixo energia": isso nos permite permanecer no âmbito das variedades simpléticas, mais especificamente do "espaço de fase duplamente estendido" $\mathbb{R} \times P_{0} \times \mathbb{R}$, já considerado por É. Cartan. Ambas as extensões são, de maneira óbvia, fibrados sobre o eixo temporal $\mathbb{R}$, e é esta a estrutura que será reproduzida naturalmente quando nossa definição de fibrado multissimplético é aplicada ao caso de uma variedade base unidimensional.

- Na mecânica clássica relativística a noção de tempo sofre uma mudança radical de interpretação quando comparada com o caso não relativístico. Neste prevalece a noção do tempo absoluto de Newton, que pode ser usado, e é, como parâmetro universal para as soluções das equações diferenciais ordinárias para todos os sistemas dinâmicos possíveis, e de uma só vez. Na transição para o caso relativístico o tempo perde este estatus privilegiado e passa a ser uma coordenada (quase) como qualquer outra - pois afinal, coordenadas temporais e espaciais se misturam sob tranformações de Lorentz. Portanto é absolutamente natural combiná-lo com as coordenadas generalizadas das posições e dos momentos da mecânica, uma vez que já não representa uma variável independente, e sim uma variável dependente como estas, enquanto que a nova variável independente é algo diferente: para trajetórias de uma partícula é o tempo próprio medido por um relógio que se move junto a esta, ao contrário da coordenada tempo, medida em relação ao referencial de um observador distante.

Resumindo: a mecânica provê sim um contexto natural para o aparecimento de fibrados simpléticos (sobre a reta real).

Levando em conta estas considerações, podemos fazer uma segunda tentativa de definir o conceito de uma estrutura multissimplética, segundo a qual um fibrado multissimplético é um fibrado $P$ sobre uma variedade $n$-dimensional $M$ munido de uma forma diferencial $\omega$ de grau $n+1$ que é fechada $(d \omega=0)$ assim como não degenerada e $(n-1)$-horizontal, i.e., 
satisfaz a equação (5) como antes e

$$
\mathrm{i}_{v_{1}} \mathrm{i}_{v_{2}} \mathrm{i}_{v_{3}} \omega(p)=0 \quad \text { para } p \in P, v_{1}, v_{2}, v_{3} \in V_{p} P
$$

onde $V P$ denota o fibrado vertical ( $V P=\operatorname{ker} T \pi \operatorname{com} T \pi: T P \rightarrow T M$ a aplicação tangente à projeção $\pi: P \rightarrow M)$. Como veremos, estas condições nos permitem

- Introduzir um "núcleo vertical comum" de $\omega$, que é uma distribuição $K_{\omega}$ involutiva contida em $V P$, definida por

$$
\left(K_{\omega}\right)_{p} P=\left\{v \in V_{p} P \mid \mathrm{i}_{v^{\prime}} \mathrm{i}_{v^{\prime}} \omega(p)=0 \text { para todo } v^{\prime} \in V_{p} P\right\}
$$

e que será, no máximo, unidimensional: pode ser trivial, como no caso do "espaço de fase simplesmente estendido" da mecânica, ou pode ser unidimensional, como no caso do "espaço de fase duplamente estendido" da mecânica, onde representa a variável adicional energia.

- Reduzir a forma "multissimplética" original $\omega$ a uma forma "polissimplética" $\hat{\omega}$ sobre o fibrado vertical (i.e., uma família de formas "polissimpléticas" $\hat{\omega}_{x}$ sobre as fibras $P_{x}$, parametrizadas pelos pontos $x$ do espaço base $M$ ), configurando um homomorfismo

$$
\hat{\omega}: \bigwedge^{2} V P \longrightarrow \pi^{*}\left(\bigwedge^{n-1} T^{*} M\right)
$$

de fibrados vetoriais sobre $P$ para o qual $K P$ é o "núcleo comum" destas.

Infelizmente, estas exigências ainda não são suficientes para garantir a existência de coordenadas canônicas (locais). De fato, existem contraexemplos simples que mostram que algo importante está faltando: é preciso incluir uma condição algébrica adicional que nos permita encontrar uma base de Darboux no espaço tangente de cada ponto. Um segundo problema é então analisar se (ou sob quais condições) é possível encontrar bases de Darboux que (localmente) formem referenciais móveis holônomos.

Como veremos no trabalho que se segue, ambas as questões têm respostas simples e elegantes, providenciando então uma nova e finalmente adequada definição da noção de uma estrutura multissimplética. Esperamos que esta clarificação conceitual abra as portas para novos caminhos matemáticos que prometem ser altamente relevantes para a física.

Quanto à organização do trabalho, o primeiro capítulo é puramente algébrico enquanto que o segundo tem natureza geométrica. Em nível algébrico, a nova estrutura matemática que apareceu em função da nossa investigação e que é bastante geral, pois se aplica a toda uma gama de situações diferentes, pode ser resumida como "existência de um tipo especial de subespaço isotrópico maximal" que chamamos de "subespaço polilagrangeano" ou "subespaço multilagrangeano", dependendo do contexto. Este conceito torna viável o tratamento 
de formas mais gerais do que as polissimpléticas ou multissimpléticas e que nós chamamos de "polilagrangeanas" ou "multilagrangeanas", respectivamente. Revela-se que estas constituem o arcabouço adequado para a formulação de um teorema de Darboux em nível algébrico. Subsequentemente, quando passamos do contexto puramente algébrico para o ambiente da geometria diferencial, percebe-se mais uma vez a adequação deste novo conceito algébrico, pois a sua combinação com propriedades clássicas de integrabilidade $(d \omega=0)$ permite obter o teorema de Darboux no contexto geométrico (existência de coordenadas locais canônicas). No terceiro capítulo, iniciamos a análise das consequências dos resultados obtidos para o caso específico de estruturas polissimpléticas e multissimpléticas e também comparamos a nossa abordagem com a de outros autores. 
CaPÍTUlo 1

\section{Formas polilagrangeanas e multilagrangeanas: teoria algébrica}

Neste capítulo investigamos duas generalizações de formas multilineares alternadas em espaços vetoriais que desempenham um papel importante na teoria clássica dos campos e que chamaremos de formas polilagrangeanas (contendo como caso especial as formas polissimpléticas) e de formas multilagrangeanas (contendo como caso especial as formas multissimpléticas), sendo as primeiras formas a valores vetoriais e as segundas formas parcialmente horizontais, i.e., formas comuns com um certo grau de horizontalidade em relação a um subespaço "vertical" fixo. Começamos pela definição de algumas noções gerais, tais como a de núcleo, de suporte e de posto, e logo passamos a introduzir vários tipos de complementos ortogonais a serem usados na definição de certas classes de subespaços que generalizam os subespaços isotrópicos, coisotrópicos, lagrangeanos e maximais isotrópicos da geometria simplética às situações sob estudo aqui. Como um dos pontos centrais desta tese, mostraremos que existe uma extensão específica do conceito de subespaço lagrangeano que chamaremos de subespaço polilagrangiano (no caso das formas a valores vetoriais) ou multilagrangeano (no caso das formas parcialmente horizontais) e que permite desenvolver uma teoria não-trivial de formas polissimpléticas e multissimpléticas que abrange tanto as situações estudadas na literatura matemática existente como as situações relevantes para as aplicações em física, que ainda não foram adequadamente contempladas.

A primeira seção deste capítulo será dedicada ao estudo de algumas propriedades gerais de formas a valores vetoriais. Inicialmente, lembremos que dados dois espaços vetoriais $V$ e $\hat{T}$, ambos de dimensão finita ${ }^{1}$, uma $k$-forma em $V$ a valores em $\hat{T}$ é simplesmente uma

\footnotetext{
${ }^{1}$ Neste trabalho, a hipótese de que todos os espaços vetoriais considerados sejam de dimensão finita é imposta para simplificar a apresentação das idéias centrais, mas não é impreescindível pois pode ser substituída por condições adequadas de análise funcional, que deixaremos para uma possível análise posterior.
} 
aplicação linear

$$
\hat{\omega}: \bigwedge^{k} V \longrightarrow \hat{T}
$$

ou, em outras palavras, uma aplicação $k$-multilinear alternada de $V \times \ldots \times V$ ( $k$ fatores) em $\hat{T}$. O espaço vetorial destas formas será denotado por

$$
L_{a}^{k}(V ; \hat{T}) \cong L\left(\bigwedge^{k} V ; \hat{T}\right) \cong\left(\bigwedge^{k} V^{*}\right) \otimes \hat{T}
$$

Se tomarmos $\hat{T}=\mathbb{R}$ temos reconstituída a noção de uma $k$-forma comum em $V$.

A segunda seção deste capítulo será dedicada a certos tipos de formas parcialmente horizontais. Ressaltamos que para podermos falar de horizontalidade é preciso fixar um espaço vetorial $W$ em conjunto com um subespaço $V$, chamado de subespaço vertical, de modo que denotando o espaço quociente $W / V$, chamado de espaço base, por $T$ e a projeção canônica de $W$ em $T$ por $\pi$, obtemos a seguinte sequência exata de espaços vetoriais:

$$
0 \longrightarrow V \longrightarrow W \stackrel{\pi}{\longrightarrow} T \longrightarrow 0 \text {. }
$$

Lembramos que uma $k$-forma $\alpha \in \bigwedge^{k} W^{*}$ em $W$ é dita horizontal (relativo a $\pi$ ) se a contração de $\alpha$ com qualquer vetor em $V=\operatorname{ker} \pi$ se anula [10, Vol. 2], [16, Vol. 1]. Mais geralmente, uma $k$-forma $\alpha \in \bigwedge^{k} W^{*}$ em $W$ é dita $(k-r)$-horizontal (relativo a $\pi$ ), onde $0 \leqslant r \leqslant k$, se a contração de $\alpha$ com mais de $r$ vetores em $V=\operatorname{ker} \pi$ se anula:

$$
\mathrm{i}_{v_{1}} \ldots \mathrm{i}_{v_{r+1}} \alpha=0 \quad \text { para } v_{1}, \ldots, v_{r+1} \in V
$$

O espaço vetorial das $k$-formas $(k-r)$-horizontais em $W$ será denotado por $\bigwedge_{r}^{k} W^{*}$. Obviamente, a condição de 0-horizontalidade $(r=k)$ é vazia, e assim vale $\bigwedge_{k}^{k} W^{*}=\bigwedge^{k} W^{*}$, enquanto que formas $k$-horizontais são simplesmente formas horizontais, e temos um isomorfismo canônico $\bigwedge_{0}^{k} W^{*} \cong \bigwedge^{k} T^{*}$. Para uso posterior, notamos que na direção $\leftarrow$, i.e., como aplicação linear

$$
\begin{aligned}
\bigwedge^{k} T^{*} & \longrightarrow \bigwedge_{0}^{k} W^{*} \\
\alpha_{T} & \longmapsto \alpha_{W}
\end{aligned}
$$

ele pode ser explicitamente definido como o pull-back pela projeção $\pi$, i.e., por $\alpha_{W}=\pi^{*}\left(\alpha_{T}\right)$, ou seja,

$$
\alpha_{W}\left(w_{1}, \ldots, w_{k}\right)=\alpha_{T}\left(\pi\left(w_{1}\right), \ldots, \pi\left(w_{k}\right)\right) \quad \text { para } w_{1}, \ldots, w_{k} \in W
$$

Além disso, temos a sequência de inclusões

$$
\bigwedge^{k} T^{*} \cong \bigwedge_{0}^{k} W^{*} \subset \ldots \subset \bigwedge_{r}^{k} W^{*} \subset \ldots \subset \bigwedge_{k}^{k} W^{*}=\bigwedge^{k} W^{*}
$$


onde alguns dos primeiros termos podem ser triviais, pois

$$
\bigwedge_{r}^{k} W^{*}=\{0\} \quad \text { se } k-r>\operatorname{dim} T .
$$

Assim, motiva-se a interpretação destas formas como "parcialmente horizontais". Mais exatamente, podemos justificar essa terminologia observando que se introduzirmos uma base $\left\{e_{1}^{V}, \ldots, e_{m}^{V}, e_{1}^{T}, \ldots, e_{n}^{T}\right\}$ de $W$ tal que os primeiros $m$ vetores formam uma base de $V$ enquanto que os últimos $n$ vetores formam uma base de um subespaço complementar a $V$, isomorfo a $T$, então em termos da base dual $\left\{e_{V}^{1}, \ldots, e_{V}^{m}, e_{T}^{1}, \ldots, e_{T}^{n}\right\}$ de $W^{*}$, qualquer forma $\alpha \in \bigwedge_{r}^{k} W^{*}$ admite a expansão

$$
\alpha=\sum_{s=0}^{r} \frac{1}{s !} \frac{1}{(k-s) !} \alpha_{i_{1} \ldots i_{s} ; \mu_{1} \ldots \mu_{k-s}} e_{V}^{i_{1}} \wedge \ldots \wedge e_{V}^{i_{s}} \wedge e_{T}^{\mu_{1}} \wedge \ldots \wedge e_{T}^{\mu_{k-s}}
$$

mostrando que formas $\alpha \in \bigwedge_{r}^{k} W^{*}$ são $(k-r)$-horizontais no sentido de poderem ser expressas como combinações lineares de produtos exteriores de 1-formas entre as quais pelo menos $k-r$ são horizontais, i.e., pertencem ao subespaço $V^{\perp}$ (que tem base $\left.\left\{e_{T}^{1}, \ldots, e_{T}^{n}\right\}\right){ }^{2}$ Isso também significa que $\left\{e_{V}^{i_{1}} \wedge \ldots \wedge e_{V}^{i_{s}} \wedge e_{T}^{\mu_{1}} \wedge \ldots \wedge e_{T}^{\mu_{k-s}} \mid 0 \leqslant s \leqslant r, 1 \leqslant i_{1}<\ldots<i_{s} \leqslant \operatorname{dim} V\right.$, $\left.1 \leqslant \mu_{1}<\ldots<\mu_{k-s} \leqslant \operatorname{dim} T\right\}$ é uma base de $\bigwedge_{r}^{k} W^{*}$ e portanto vale

$$
\operatorname{dim} \bigwedge_{r}^{k} W^{*}=\sum_{s=0}^{r}\left(\begin{array}{c}
\operatorname{dim} V \\
s
\end{array}\right)\left(\begin{array}{c}
\operatorname{dim} T \\
k-s
\end{array}\right)
$$

onde é subentendido que, por definição, $\left(\begin{array}{l}p \\ q\end{array}\right)=0$ se $q>p$.

$\mathrm{Na}$ terceira seção faremos uso de uma construção que chamaremos de símbolo e que permite associar a cada forma parcialmente horizontal uma certa forma a valores vetoriais. Este procedimento está por trás do elo entre estruturas multissimpléticas e estruturas polissimpléticas. Na notação do parágrafo anterior, o símbolo de uma $k$-forma $\alpha$ $(k-r)$-horizontal em $W, \alpha \in \bigwedge_{r}^{k} W^{*}$, é a $r$-forma $\hat{\alpha}$ em $V$ a valores no espaço $\bigwedge^{k-r} T^{*}$, $\hat{\alpha} \in \bigwedge^{r} V^{*} \otimes \bigwedge^{k-r} T^{*}$, definida por

$$
\hat{\alpha}\left(v_{1}, \ldots, v_{r}\right)=\mathrm{i}_{v_{1}} \ldots \mathrm{i}_{v_{r}} \alpha, \quad \text { para } v_{1}, \ldots, v_{r} \in V .
$$

onde fazemos uso das identificações

$$
L_{a}^{r}\left(V ; \bigwedge^{k-r} T^{*}\right) \cong L\left(\bigwedge^{r} V ; \bigwedge^{k-r} T^{*}\right) \cong \bigwedge^{r} V^{*} \otimes \bigwedge^{k-r} T^{*},
$$

$\mathrm{e}$

$$
\bigwedge^{k-r} T^{*} \cong \bigwedge_{0}^{k-r} W^{*}
$$

\footnotetext{
${ }^{2}$ Neste trabalho, usamos o símbolo.$^{\perp}$ para denotar o aniquilador, i.e., $V^{\perp}$ é o subespaço de $W^{*}$ consistindo de todas as formas lineares sobre $W$ que se anulam sobre $V$.
} 
Para entender melhor esta construção, introduzimos uma base $\left\{e_{1}^{V}, \ldots, e_{m}^{V}, e_{1}^{T}, \ldots, e_{n}^{T}\right\}$ de $W$ com base dual $\left\{e_{V}^{1}, \ldots, e_{V}^{m}, e_{T}^{1}, \ldots, e_{T}^{n}\right\}$ de $W^{*}$ como antes; então se a forma $\alpha \in \bigwedge_{r}^{k} W^{*}$ é dada pela expansão

$$
\alpha=\sum_{s=0}^{r} \frac{1}{s !} \frac{1}{(k-s) !} \alpha_{i_{1} \ldots i_{s} ; \mu_{1} \ldots \mu_{k-s}} e_{V}^{i_{1}} \wedge \ldots \wedge e_{V}^{i_{s}} \wedge e_{T}^{\mu_{1}} \wedge \ldots \wedge e_{T}^{\mu_{k-s}}
$$

a forma $\hat{\alpha} \in \bigwedge^{r} V^{*} \otimes \bigwedge^{k-r} T^{*}$ é dada pela expansão

$$
\hat{\alpha}=\frac{1}{r !} \frac{1}{(k-r) !} \alpha_{i_{1} \ldots i_{r} ; \mu_{1} \ldots \mu_{k-r}} e_{V}^{i_{1}} \wedge \ldots \wedge e_{V}^{i_{r}} \otimes e_{T}^{\mu_{1}} \wedge \ldots \wedge e_{T}^{\mu_{k-r}} .
$$

Assim a passagem ao símbolo pode ser vista como uma projeção

$$
\begin{array}{ccc}
\bigwedge_{r}^{k} W^{*} & \longrightarrow & \bigwedge^{r} V^{*} \otimes \bigwedge^{k-r} T^{*} \\
\alpha & \longmapsto & \hat{\alpha}
\end{array}
$$

cujo núcleo é o subespaço $\bigwedge_{r-1}^{k} W^{*}$.

Uma ferramenta auxiliar muito utilizada no contexto descrito nos últimos dois parágrafos é a escolha de uma cisão da sequência exata (1.3), i.e., de uma aplicação linear $\mathfrak{s}: T \rightarrow W$ tal que $\pi \circ \mathfrak{s}=\mathrm{id}_{T}$, ou equivalentemente, a escolha de um subespaço $H$ de $W$, chamado de subespaço horizontal, que é complementar a $V$, i.e., que satisfaz

$$
W=V \oplus H,
$$

sendo que $H$ é simplesmente a imagem de $\mathfrak{s}$ em $W$ e $\mathfrak{s}$ é simplesmente o inverso da restrição da projeção $\pi$ a $H$. Então é fácil ver que

$$
W^{*}=H^{\perp} \oplus V^{\perp},
$$

sendo que $H^{\perp} \cong V^{*}$ e $V^{\perp} \cong H^{*}$, e mais geralmente

$$
\bigwedge_{r}^{k} W^{*} \cong \bigoplus_{s=0}^{r} \bigwedge^{s} H^{\perp} \otimes \bigwedge^{k-s} V^{\perp}
$$

Voltando ao isomorfismo canônico $\bigwedge_{0}^{k} W^{*} \cong \bigwedge^{k} T^{*}$, notamos que na direção $\rightarrow$, i.e., como aplicação linear

$$
\begin{array}{ccc}
\bigwedge_{0}^{k} W^{*} & \longrightarrow \bigwedge^{k} T^{*} \\
\alpha_{W} & \longmapsto \alpha_{T}
\end{array}
$$

ele pode ser explicitamente definido como o pull-back pela cisão $\mathfrak{s}$, i.e., por $\alpha_{T}=\mathfrak{s}^{*}\left(\alpha_{W}\right)$, ou seja,

$$
\alpha_{T}\left(t_{1}, \ldots, t_{k}\right)=\alpha_{W}\left(\mathfrak{s}\left(t_{1}\right), \ldots, \mathfrak{s}\left(t_{k}\right)\right) \quad \text { para } t_{1}, \ldots, t_{k} \in T
$$


Logo chegamos à seguinte fórmula explícita para o símbolo $\hat{\alpha} \in \bigwedge^{r} V^{*} \otimes \bigwedge^{k-r} T^{*}$ de uma forma parcialmente horizontal $\alpha \in \bigwedge_{r}^{k} W^{*}$ :

$$
\begin{gathered}
\hat{\alpha}\left(v_{1}, \ldots, v_{r}\right)\left(t_{1}, \ldots, t_{k-r}\right)=\alpha\left(v_{1}, \ldots, v_{r}, \mathfrak{s}\left(t_{1}\right), \ldots, \mathfrak{s}\left(t_{k-r}\right)\right) \\
\operatorname{para} v_{1}, \ldots, v_{r} \in V, t_{1}, \ldots, t_{k-r} \in T
\end{gathered}
$$

No entanto, cabe enfatizar que as últimas duas fórmulas, assim como todas as construções descritas nos dois parágrafos anteriores, não dependem da escolha da cisão $\mathfrak{s}$.

$\mathrm{Na}$ quarta seção apresentamos a construção do que podemos considerar o exemplo canônico de uma forma polilagrangeana e de uma forma multilagrangeana. Finalmente, nas últimas duas seções, mostraremos que formas polilagrangeanas e formas multilagrangeanas admitem uma "forma padrão" deduzida do exemplo canônico da seção anterior. Expressa em termos de bases adaptadas, esta afirmação pode ser considerada um "precursor algébrico" do teorema de Darboux.

No que segue, adotaremos a convenção geral de que objetos ligados a formas a valores vetoriais serão caracterizados pelo símbolo de um chapéu, como acima. Ademais, como estamos interessados apenas em formas de grau $k>1$, efetuaremos uma mudança do parâmetro $k$, passando a considerar $(k+1)$-formas $\omega \in \bigwedge^{k+1} W^{*}$ comuns e $(k+1)$-formas

$\hat{\omega} \in \bigwedge^{k+1} V^{*} \otimes \hat{T}$ a valores vetoriais, com $k \geqslant 1$. Finalmente, a dimensão dos espaços "auxiliares" $T$ e $\hat{T}$ será denotada por $n$ e por $\hat{n}$, respectivamente.

Todos os resultados deste capítulo são formulados para espaços vetoriais sobre o corpo $\mathbb{R}$ dos números reais, mas permanecem válidos para espaços vetoriais sobre qualquer corpo de característica 0.

\subsection{Formas a valores vetoriais em espaços vetoriais}

Nesta seção apresentamos alguns conceitos gerais que podem ser estendidos de formas comuns para o contexto mais geral de formas a valores vetoriais sem dificuldade alguma. Também introduzimos um tipo especial de subespaço maximal isotrópico, ou lagrangeano, chamado de subespaço polilagrangeano, que desempenha um papel fundamental na teoria das formas polissimpléticas, a ser desenvolvida no próximo capítulo.

Sejam $V$ e $\hat{T}$ espaços vetoriais de dimensão finita, com $\operatorname{dim} \hat{T}=\hat{n}$, e seja $\hat{\omega}$ uma $(k+1)$ forma em $V$ a valores em $\hat{T}$ :

$$
\hat{\omega} \in \bigwedge^{k+1} V^{*} \otimes \hat{T} .
$$

O núcleo de $\hat{\omega}$ é o subespaço ker $\hat{\omega}$ de $V$ definido por

$$
\text { ker } \hat{\omega}=\left\{v \in V \mid \mathrm{i}_{v} \hat{\omega}=0\right\} \text {, }
$$


ou seja,

$$
\text { ker } \hat{\omega}=\left\{v \in V \mid \hat{\omega}\left(v, v_{1}, \ldots, v_{k}\right)=0 \text { para todo } v_{1}, \ldots, v_{k} \in V\right\} .
$$

Quando ker $\hat{\omega}=0$ dizemos que $\hat{\omega}$ é não-degenerada. O suporte de $\hat{\omega}$ é o subespaço supp $\hat{\omega}$ de $V^{*}$ definido como o aniquilador de ker $\hat{\omega}$, quer dizer, ${ }^{3}$

$$
\operatorname{supp} \hat{\omega}=(\operatorname{ker} \hat{\omega})^{\perp}=\left\{v^{*} \in V^{*} \mid\left\langle v^{*}, v\right\rangle=0 \text { para todo } v \in \operatorname{ker} \hat{\omega}\right\} \text {. }
$$

Reciprocamente temos

$$
\text { ker } \hat{\omega}=(\operatorname{supp} \hat{\omega})^{\perp}=\left\{v \in V \mid\left\langle v^{*}, v\right\rangle=0 \text { para todo } v^{*} \in \operatorname{supp} \hat{\omega}\right\} \text {. }
$$

O posto $\operatorname{rk}(\hat{\omega})$ de $\hat{\omega}$, segundo alguns autores (veja, por exemplo, Ref. [18]), é a dimensão do seu suporte:

$$
\operatorname{rk}(\hat{\omega})=\operatorname{dim} \operatorname{supp} \hat{\omega}
$$

No entanto, dependendo da natureza específica da forma $\hat{\omega}$, pode ser conveniente adotar uma definição numericamente diferente, como já acontece no caso elementar das formas simpléticas em espaços vetoriais de dimensão $2 N$, cujo posto costuma ser definido como $N$ e não como $2 N$. O mesmo tipo de fenômeno aparecerá no caso das diversas formas estudadas neste trabalho.

Uma ferramenta útil no estudo de formas a valores vetoriais é que podemos considerálas como "multipletos" de formas comuns. Mais especificamente, podemos a cada forma linear $\hat{t}^{*} \in \hat{T}^{*}$ no espaço auxiliar $\hat{T}$ associar uma $(k+1)$-forma comum $\omega_{\hat{t}^{*}}$ em $V$ chamada a projeção de $\hat{\omega}$ ao longo de $\hat{t}^{*}$ e definida por

$$
\omega_{\hat{t}^{*}}=\left\langle\hat{t}^{*}, \hat{\omega}\right\rangle \text {. }
$$

Obviamente, $\omega_{\hat{t}^{*}}$ depende linearmente de $\hat{t}^{*}$ e portanto se tomarmos uma base $\left\{\hat{e}_{1}, \ldots, \hat{e}_{\hat{n}}\right\}$ de $\hat{T}$, com base dual $\left\{\hat{e}^{1}, \ldots, \hat{e}^{\hat{n}}\right\}$ de $\hat{T}^{*}$, e definirmos as $(k+1)$-formas comuns

$$
\omega^{a}=\omega_{\hat{e}^{a}} \quad(1 \leqslant a \leqslant \hat{n})
$$

temos

$$
\hat{\omega}=\omega^{a} \otimes \hat{e}_{a}
$$

Então fica claro que

$$
\operatorname{ker} \hat{\omega}=\bigcap_{\hat{t}^{*} \in \hat{T}^{*}} \operatorname{ker} \omega_{\hat{t}^{*}}
$$

\footnotetext{
${ }^{3}$ Neste trabalho, usamos o símbolo $\langle.,$.$\rangle para denotar o pareamento bilinear natural entre um espaço$ vetorial e seu dual.
} 
ou ainda

$$
\operatorname{ker} \hat{\omega}=\bigcap_{a=1}^{\hat{n}} \operatorname{ker} \omega^{a} \text {, }
$$

e tomando o aniquilador, que

$$
\operatorname{supp} \hat{\omega}=\sum_{\hat{t}^{*} \in \hat{T}^{*}} \operatorname{supp} \omega_{\hat{t}^{*}},
$$

ou ainda

$$
\operatorname{supp} \hat{\omega}=\sum_{a=1}^{\hat{n}} \operatorname{supp} \omega^{a} .
$$

A seguinte caracterização do suporte é frequentemente usada como definição:

Lema 1.1 O suporte supp $\hat{\omega}$ de $\hat{\omega}$ é o menor subespaço de $V^{*}$ tal que vale

$$
\hat{\omega} \in\left(\bigwedge^{k+1} \operatorname{supp} \hat{\omega}\right) \otimes \hat{T}
$$

DemonstraÇÃo. Usando uma base $\left\{\hat{e}_{1}, \ldots, \hat{e}_{\hat{n}}\right\}$ de $\hat{T}$ como antes, em conjunto com uma base $\left\{e_{1}, \ldots, e_{r}\right\}$ de $V$ composta de uma base $\left\{e_{1}, \ldots, e_{s}\right\}$ de ker $\hat{\omega}$ seguida de uma base $\left\{e_{s+1}, \ldots, e_{r}\right\}$ de um subespaço de $V$ complementar a ker $\hat{\omega}$ e considerando a correspondente base dual $\left\{e^{1}, \ldots, e^{r}\right\}$ de $V^{*}$, vemos que $\left\{e^{s+1}, \ldots, e^{r}\right\}$ é uma base de supp $\hat{\omega}=(\text { ker } \hat{\omega})^{\perp}$, e expandindo $\hat{\omega}$ em termos da base $\left\{e^{p_{1}} \wedge \ldots \wedge e^{p_{k+1}} \otimes \hat{e}_{a} \mid 1 \leqslant p_{1}<\ldots<p_{k+1} \leqslant r, 1 \leqslant a \leqslant \hat{n}\right\}$ de $\bigwedge^{k+1} V^{*} \otimes \hat{T}$, vemos que todos os coeficientes $\omega_{p_{1} \ldots p_{k+1}}^{a}$ com $p_{1} \leqslant s$ se anulam, o que implica que $\hat{\omega} \in\left(\bigwedge^{k+1} \operatorname{supp} \hat{\omega}\right) \otimes \hat{T}$. Por outro lado, se $S_{\hat{\omega}}$ é qualquer subespaço de $V^{*}$ tal que vale $\hat{\omega} \in\left(\bigwedge^{k+1} S_{\hat{\omega}}\right) \otimes \hat{T}$, então para $v \in S_{\hat{\omega}}^{\perp}$ temos $\mathrm{i}_{v} \hat{\omega}=0$ e portanto $v \in$ ker $\hat{\omega}$, ou seja, $S_{\hat{\omega}}^{\perp} \subset \operatorname{ker} \hat{\omega} ; \log \mathrm{o}, \operatorname{supp} \hat{\omega}=(\operatorname{ker} \hat{\omega})^{\perp} \subset S_{\hat{\omega}}$.

Uma noção de importância central para este trabalho é a do complemento ortogonal de um subespaço dado, que para formas de grau maior do que $2(k>1)$ vem em várias variantes e, para formas comuns, já aparece em [3]. Mais precisamente, dado um subespaço $L$ de $V$ e um inteiro $\ell$ com $1 \leqslant \ell \leqslant k$, definimos o complemento $\ell$-ortogonal de $L$ (relativo a $\hat{\omega}$ ) como o subespaço $L^{\hat{\omega}, \ell}$ de $V$ definido por ${ }^{4}$

$$
L^{\hat{\omega}, \ell}=\left\{v \in V \mid \mathrm{i}_{v} \mathrm{i}_{v_{1}} \ldots \mathrm{i}_{v_{\ell}} \hat{\omega}=0 \text { para todo } v_{1}, \ldots, v_{\ell} \in L\right\} .
$$

\footnotetext{
${ }^{4}$ Descartamos o caso trivial $\ell=0$, pois extrapolando a definição aqui adotada, $L^{\hat{\omega}, 0}$ seria simplesmente o núcleo de $\hat{\omega}$, independentemente da escolha do subespaço $L$ de $V$.
} 
Note a sequência de inclusões

$$
L^{\hat{\omega}, 1} \subset \ldots \subset L^{\hat{\omega}, \ell-1} \subset L^{\hat{\omega}, \ell} \subset L^{\hat{\omega}, \ell+1} \subset \ldots \subset L^{\hat{\omega}, k} .
$$

Como no caso de 2-formas, a noção de complemento ortogonal pode ser usado para definir subespaços isotrópicos, coisotrópicos e lagrangeanos:

- $L$ é chamado $\ell$-isotrópico (relativo a $\hat{\omega}$ ) se $L \subset L^{\hat{\omega}, \ell}$;

- $L$ é chamado $\ell$-coisotrópico (relativo a $\hat{\omega}$ ) se $L \supset L^{\hat{\omega}, \ell}$;

- $L$ é chamado $\ell$-lagrangeano (relativo a $\hat{\omega}$ ) se for simultaneamente $\ell$-isotrópico e $\ell$-coisotrópico, ou seja, se $L=L^{\hat{\omega}, \ell}$.

Entre os subespaços $\ell$-isotrópicos, destacam-se os maximais, definidos de modo padrão: $L$ é chamado maximal $\ell$-isotrópico (relativo a $\hat{\omega}$ ) se $L$ é $\ell$-isotrópico (relativo a $\hat{\omega}$ ) e se para qualquer outro subespaço $L^{\prime}$ de $V$ que também é $\ell$-isotrópico (relativo a $\hat{\omega}$ ), vale

$$
L \subset L^{\prime} \Longrightarrow L=L^{\prime}
$$

Observamos que subespaços $\ell$-lagrangeanos e subespaços maximais $\ell$-isotrópicos sempre contêm o núcleo de $\hat{\omega}$.

O seguinte teorema garante que subespaços maximais isotrópicos são idênticos a subespaços lagrangeanos e estabelece relações entre subespaços isotrópicos e maximais isotrópicos relativo a uma forma a valores vetoriais e relativo às formas projetadas:

Teorema 1.1 Sejam $V$ e $\hat{T}$ espaços vetoriais de dimensão finita e seja $\hat{\omega}$ uma $(k+1)$-forma em $V$ a valores em $\hat{T}$. Para um subespaço $L$ de $V$ e $1 \leqslant \ell \leqslant k$, vale

1. L é maximal $\ell$-isotrópico relativo a $\hat{\omega}$ se e somente se $L$ é $\ell$-lagrangeano relativo a $\hat{\omega}$ e, para todo $\hat{t}^{*} \in \hat{T}^{*}, L$ é maximal $\ell$-isotrópico relativo a $\hat{\omega}_{\hat{t}^{*}}$ se e somente se $L$ é

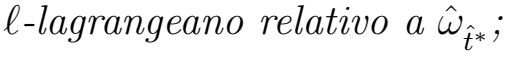

2. L é $\ell$-isotrópico relativo a $\hat{\omega}$ se e somente se para todo $\hat{t}^{*} \in \hat{T}^{*} L$ é $\ell$-isotrópico relativo $a \hat{\omega}_{\hat{t}^{*}} ;$

3. Se $L$ é maximal $\ell$-isotrópico relativo a $\hat{\omega}_{\hat{t}^{*}}$ para todo $\hat{t}^{*} \in \hat{T}^{*}$ então $L$ é maximal $\ell$ isotrópico relativo a $\hat{\omega}$.

Enfatizamos desde já que o recíproco da última afirmação deste teorema não é válido, pois um subespaço maximal isotrópico será isotrópico relativo a cada uma das formas projetadas mas pode deixar de ser maximal isotrópico relativo a algumas delas. 


\section{DEMONSTRAÇÃO.}

1. Se $L$ é $\ell$-isotrópico então para todo vetor $u \in L^{\hat{\omega}, \ell} \backslash L$, o subespaço $L^{\prime}=L+\langle u\rangle$ de $V$ também é $\ell$-isotrópico, pois para $u_{i}=v_{i}+\lambda_{i} u \in L^{\prime} \operatorname{com} v_{i} \in L(i=0, \ldots, \ell)$, temos

$$
\mathrm{i}_{u_{0}} \mathrm{i}_{u_{1}} \ldots \mathrm{i}_{u_{\ell}} \hat{\omega}=\sum_{i=0}^{\ell} \mathrm{i}_{v_{0}} \mathrm{i}_{v_{1}} \ldots \mathrm{i}_{v_{\ell}} \hat{\omega}+\sum_{i=0}^{\ell}(-1)^{i} \lambda_{i} \mathrm{i}_{u} \mathrm{i}_{v_{0}} \ldots \widehat{\mathrm{i}}_{v_{i}} \ldots \mathrm{i}_{v_{\ell}} \hat{\omega}=0 .
$$

Portanto, se $L$ é maximal $\ell$-isotrópico então $L^{\prime} \subset L$; $\operatorname{logo}, L^{\hat{\omega}, \ell} \subset L$ e assim $L$ é $\ell$ lagrangeano. Reciprocamente, se $L$ é $\ell$-lagrangeano e $L^{\prime}$ é $\ell$-isotrópico com $L \subset L^{\prime}$ então

$$
L^{\prime} \subset\left(L^{\prime}\right)^{\hat{\omega}, \ell} \subset L^{\hat{\omega}, \ell}=L,
$$

ou seja, $L$ é maximal $\ell$-isotrópico. O mesmo argumento pode ser aplicado a cada uma das formas projetadas $\hat{\omega}_{\hat{t}^{*}}\left(\hat{t}^{*} \in \hat{T}^{*}\right)$, em vez de $\hat{\omega}$.

2. Para $v_{i} \in L(i=0, \ldots, \ell)$ temos

$$
\mathrm{i}_{u_{0}} \ldots \mathrm{i}_{u_{\ell}} \hat{\omega}=0 \quad \Longleftrightarrow \quad \mathrm{i}_{u_{0}} \ldots \mathrm{i}_{u_{\ell}} \hat{\omega}_{\hat{t}^{*}}=0 \quad \text { para todo } \hat{t}^{*} \in \hat{T}^{*} .
$$

3. Suponha que $L$ é maximal $\ell$-isotrópico relativo a cada uma das formas projetadas $\hat{\omega}_{\hat{t}^{*}}$ $\left(\hat{t}^{*} \in \hat{T}^{*}\right)$. Conforme o segundo item, $L$ é $\ell$-isotrópico relativo a $\hat{\omega}$. Ademais, se $L^{\prime}$ é um subespaço de $V$ que é $\ell$-isotrópico relativo a $\hat{\omega}$ e tal que $L \subset L^{\prime}$, então conforme o segundo item, $L^{\prime}$ é $\ell$-isotrópico relativo a cada uma das formas projetadas $\hat{\omega}_{\hat{t}^{*}}\left(\hat{t}^{*} \in \hat{T}^{*}\right)$ e portanto $L^{\prime}=L$, provando que $L$ é de fato maximal $\ell$-isotrópico relativo a $\hat{\omega}$.

No que segue, estaremos interessados predominantemente no caso $\ell=1$ e, para simplificar a notação, omitiremos o prefixo "1" sempre que for conveniente, chamando subespaços (maximais) 1-isotrópicos simplesmente de (maximais) isotrópicos.

Uma outra maneira de abordar os conceitos de subespaço isotrópico e de subespaço maximal isotrópico que se revela bastante frutífera é baseada na idéia de contrair $\hat{\omega}$ com vetores. Para tanto, definimos a contração com $\hat{\omega}$ como a aplicação linear

$$
\begin{array}{ccc}
\hat{\omega}^{b}: V & \longrightarrow\left(\bigwedge^{k} V^{*}\right) \otimes \hat{T} \\
v & \longmapsto \\
\mathrm{i}_{v} \hat{\omega}
\end{array} .
$$

Então um subespaço $L$ de $V$ é isotrópico se e somente se vale a inclusão

$$
\hat{\omega}^{b}(L) \subset\left(\bigwedge^{k} L^{\perp}\right) \otimes \hat{T},
$$


que podemos, de modo trivial, reescrever na forma

$$
\hat{\omega}^{b}(L) \subset \hat{\omega}^{b}(V) \cap\left(\bigwedge^{k} L^{\perp}\right) \otimes \hat{T}
$$

O mérito desta aparentemente inútil reformulação consiste na analogia com o critério de que um subespaço $L$ de $V$ que contém ker $\hat{\omega}^{b}=\operatorname{ker} \hat{\omega}$ é maximal isotrópico se e somente se vale a igualdade

$$
\hat{\omega}^{b}(L)=\hat{\omega}^{b}(V) \cap\left(\bigwedge^{k} L^{\perp}\right) \otimes \hat{T} .
$$

De fato, para qualquer subespaço $L$ de $V$, vale a identidade

$$
\hat{\omega}^{b}\left(L^{\hat{\omega}, 1}\right)=\hat{\omega}^{b}(V) \cap\left(\bigwedge^{k} L^{\perp}\right) \otimes \hat{T},
$$

pois ambos os lados são iguais ao subespaço de $\left(\bigwedge^{k} V^{*}\right) \otimes \hat{T}$ formado pelos elementos da forma $\mathrm{i}_{v} \hat{\omega}$ com $v \in V$ e tais que $\mathrm{i}_{u} \mathrm{i}_{v} \hat{\omega}=0$ para todo $u \in L$.

Posto isso, podemos introduzir um conceito que constitui a chave mestre para o estudo de formas polissimpléticas: a existência de subespaços isotrópicos maximais "especiais", que nós chamaremos de subespaços polilagrangeanos:

Definição 1.1 Sejam $V$ e $\hat{T}$ espaços vetoriais de dimensão finita, com $\hat{n} \equiv \operatorname{dim} \hat{T}$, e seja $\hat{\omega}$ uma $(k+1)$-forma em $V$ a valores em $\hat{T}$. Dizemos que $\hat{\omega}$ é uma forma polilagrangeana de posto $N$ se $V$ admite um subespaço $L$ de codimensão $N$ que é polilagrangeano, i.e., que satisfaz

$$
\hat{\omega}^{b}(L)=\left(\bigwedge^{k} L^{\perp}\right) \otimes \hat{T} .
$$

Quando $k=1$, dizemos que $\hat{\omega}$ é uma forma polissimplética.

O seguinte teorema mostra que um subespaço polilagrangeano, quando existir, contém o núcleo de $\hat{\omega}$ e portanto realmente é um tipo especial de subespaço maximal isotrópico.

Teorema 1.2 Sejam $V$ e $\hat{T}$ espaços vetoriais de dimensão finita, com $\hat{n} \equiv \operatorname{dim} \hat{T}$, e seja $\hat{\omega}$ uma $(k+1)$-forma em $V$ a valores em $\hat{T}$ que é polilagrangeana de posto $N$, com subespaço polilagrangeano $L$. Então vale $N \geqslant k$ (exceto se $\hat{\omega} \equiv 0$ ) e $L$ contém o núcleo de $\hat{\omega}$ assim como o núcleo de cada uma das formas projetadas $\omega_{\hat{t}^{*}}\left(\hat{t}^{*} \in \hat{T}^{*} \backslash\{0\}\right)$ :

$$
\text { ker } \hat{\omega} \subset L \quad, \quad \operatorname{ker} \omega_{\hat{t}^{*}} \subset L \text { para todo } \hat{t}^{*} \in \hat{T}^{*} \backslash\{0\} \text {. }
$$

A dimensão de L é dada por

$$
\operatorname{dim} L=\operatorname{dim} \operatorname{ker} \hat{\omega}+\hat{n}\left(\begin{array}{l}
N \\
k
\end{array}\right) .
$$


DemonstraÇÃo. Primeiro, observamos que se $N<k$, temos $\bigwedge^{k} L^{\perp}=\{0\}$ e portanto os dois lados da equação (1.43) se anulam; assim, $L$ é contido em ker $\hat{\omega}$ e portanto ker $\hat{\omega}$ tem codimensão $<k$ em $V$, o que força $\hat{\omega} \equiv 0$ pois a $(k+1)$-forma no espaço quociente $V / \operatorname{ker} \hat{\omega}$ induzida por $\hat{\omega}$ se anula. (Este argumento mostra, mais geralmente, que para qualquer $(k+1)$-forma em $V$ a valores em $\hat{T}, V$ não pode conter subespaços isotrópicos de codimensão $<k$.) Supondo então que $\operatorname{dim} L^{\perp}=N \geqslant k$, podemos para todo $v \in V \backslash L$ encontrar um conjunto linearmente independente de 1 -formas $v_{1}^{*}, \ldots, v_{k}^{*} \in L^{\perp}$ tal que $\left\langle v_{1}^{*}, v\right\rangle=1$ e $\left\langle v_{i}^{*}, v\right\rangle=0$ para $i>1$. Dado $\hat{t}^{*} \in \hat{T}^{*}$ tome $\hat{t} \in \hat{T}$ tal que $\left\langle\hat{t}^{*}, \hat{t}\right\rangle=1$. Conforme a definição de subespaço polilagrangeano, existe $u \in L$ satisfazendo

$$
\mathrm{i}_{u} \hat{\omega}=v_{1}^{*} \wedge \ldots \wedge v_{k}^{*} \otimes \hat{t} \Rightarrow \mathrm{i}_{v} \mathrm{i}_{u} \omega_{\hat{t}^{*}}=v_{2}^{*} \wedge \ldots \wedge v_{k}^{*} \neq 0
$$

e portanto $v \notin \operatorname{ker} \omega_{\hat{t}^{*}}$. Logo segue que ker $\hat{\omega} \subset \operatorname{ker} \omega_{\hat{t}^{*}} \subset L$. Assim vale

$$
\operatorname{dim} L-\operatorname{dim} \operatorname{ker} \hat{\omega}=\operatorname{dim} \hat{\omega}^{b}(L)=\operatorname{dim}\left(\left(\bigwedge^{k} L^{\perp}\right) \otimes \hat{T}\right)=\hat{n}\left(\begin{array}{l}
N \\
k
\end{array}\right) .
$$

A existência de um subespaço polilagrangeano pode ser detectada por um simples critério de dimensão:

Teorema 1.3 Sejam $V$ e $\hat{T}$ espaços vetoriais de dimensão finita, com $\hat{n} \equiv \operatorname{dim} \hat{T}$, e seja $\hat{\omega}$ uma $(k+1)$-forma em $V$ a valores em $\hat{T}$. Se $L$ é um subespaço isotrópico de $V$ de codimensão $N$ contendo ker $\hat{\omega}$ e tal que

$$
\operatorname{dim} L=\operatorname{dim} \operatorname{ker} \hat{\omega}+\hat{n}\left(\begin{array}{l}
N \\
k
\end{array}\right)
$$

então L é um subespaço polilagrangeano e $\hat{\omega}$ é uma forma polilagrangeana.

DemonstraçÃo. Sendo $L$ um subespaço isotrópico de $V$ contendo ker $\hat{\omega}$, a restrição da aplicação linear $\hat{\omega}^{\text {b }}$ (veja a equação (1.38)) a $L$ leva $L$ em $\left(\bigwedge^{k} L^{\perp}\right) \otimes \hat{T}$ e induz uma aplicação linear injetora do espaço quociente $L / \operatorname{ker} \hat{\omega}$ em $\left(\bigwedge^{k} L^{\perp}\right) \otimes \hat{T}$ que, segundo a condição (1.46), é um isomorfismo linear; logo, vale a igualdade (1.43).

No caso de formas a valores vetoriais mesmo, podemos dizer muito mais:

Teorema 1.4 Sejam $V$ e $\hat{T}$ espaços vetoriais de dimensão finita, com $\hat{n} \equiv \operatorname{dim} \hat{T} \geqslant 2$, e seja $\hat{\omega}$ uma $(k+1)$-forma em $V$ a valores em $\hat{T}$ que é polilagrangeana de posto $N$, com subespaço polilagrangeano L. Então L é explicitamente dado por

$$
L=\sum_{\hat{t}^{*} \in \hat{T}^{*} \backslash\{0\}} \operatorname{ker} \omega_{\hat{t}^{*}}
$$


e portanto é unicamente determinado. Em termos de uma base $\left\{\hat{e}_{1}, \ldots, \hat{e}_{\hat{n}}\right\}$ de $\hat{T}$ com base dual $\left\{\hat{e}^{1}, \ldots, \hat{e}^{\hat{n}}\right\}$ de $\hat{T}^{*}$,

$$
L=\operatorname{ker} \hat{\omega} \oplus K_{1} \oplus \ldots \oplus K_{\hat{n}}
$$

e para todo $1 \leqslant a \leqslant \hat{n}$

$$
L=\operatorname{ker} \omega^{a} \oplus K_{a},
$$

onde para todo $1 \leqslant a \leqslant \hat{n}, K_{a}$ é um subespaço qualquer de $V$ tal que

$$
\bigcap_{\substack{b=1 \\ b \neq a}}^{\hat{n}} \operatorname{ker} \omega^{b}=\operatorname{ker} \hat{\omega} \oplus K_{a}
$$

sendo que as dimensões dos vários espaços são dadas por

$$
\operatorname{dim} \operatorname{ker} \omega^{a}=\operatorname{dim} \operatorname{ker} \hat{\omega}+(\hat{n}-1)\left(\begin{array}{l}
N \\
k
\end{array}\right), \quad \operatorname{dim} K_{a}=\left(\begin{array}{l}
N \\
k
\end{array}\right) .
$$

DemonstraÇÃo. Fixamos uma base $\left\{\hat{e}_{1}, \ldots, \hat{e}_{\hat{n}}\right\}$ de $\hat{T}$ com base dual $\left\{\hat{e}^{1}, \ldots, \hat{e}^{\hat{n}}\right\}$ de $\hat{T}^{*}$ e construímos os subespaços $K_{a}$ de $V(a=1, \ldots, \hat{n})$ como descrito acima. Então os subespaços ker $\hat{\omega}$ e $K_{1}, \ldots, K_{\hat{n}}$ de $V$ se intersectam trivialmente e portanto a sua soma é direta e define um subespaço de $V$ que (provisoriamente) denotaremos por $L^{\prime}$. Pelo teorema anterior, $L^{\prime} \subset L$. Para mostrar que $L^{\prime}=L$, basta provar que $\hat{\omega}^{b}(L) \subset \hat{\omega}^{b}\left(L^{\prime}\right)$, pois $L$ e $L^{\prime}$ contêm ker $\hat{\omega}$. Usando a definição de subespaço polilagrangeano, concluímos que precisamos provar a inclusão

$$
\left(\bigwedge^{k} L^{\perp}\right) \otimes \hat{T} \subset \hat{\omega}^{b}\left(L^{\prime}\right)
$$

Mas a igualdade (1.43) mostra que para todo $\alpha \in \bigwedge^{k} L^{\perp}$ e para todo $1 \leqslant a \leqslant \hat{n}$, existe um vetor $v_{a} \in L$ tal que

$$
\mathrm{i}_{v_{a}} \hat{\omega}=\alpha \otimes \hat{e}_{a}
$$

Como

$$
\mathrm{i}_{v_{a}} \hat{\omega}^{b}=\alpha\left\langle\hat{e}^{b}, \hat{e}_{a}\right\rangle=\delta_{a}^{b} \alpha
$$

vemos que

$$
v_{a} \in \bigcap_{\substack{b=1 \\ b \neq a}}^{\hat{n}} \operatorname{ker} \hat{\omega}^{b}
$$

Decompondo $v_{a}$ conforme a equação (1.50), encontramos um vetor $u_{a} \in K_{a}$ tal que

$$
\mathrm{i}_{u_{a}} \hat{\omega}=\alpha \otimes \hat{e}_{a}
$$


e portanto $\alpha \otimes \hat{e}_{a} \in \hat{\omega}^{b}\left(K_{a}\right) \subset \hat{\omega}^{b}\left(L^{\prime}\right)$. Finalmente, observamos que conforme a definição (1.50) dos espaços $K_{a}$, em conjunto com a relação (1.33), vale

$$
\operatorname{ker} \omega^{a} \cap K_{a}=\{0\}
$$

e portanto a equação (1.49) segue da equação (1.48). Finalmente, a fórmula (1.51) é uma consequência direta das fórmulas (1.45) ou (1.46), (1.48) e (1.49).

A unicidade do subespaço polilagrangeano, quando ele existe, vale também para formas comuns, i.e., no caso em que $\operatorname{dim} \hat{T}=1$, exceto quando $k=1$ :

Proposição 1.1 Seja $V$ um espaço vetorial de dimensão finita e seja $\hat{\omega}$ uma $(k+1)$ forma polilagrangeana de posto $N$ em $V$ a valores reais, com subespaço polilagrangeano $L$. Se $k>1$, então L é único.

Uma demonstração pode ser encontrada em [19], sendo que a hipótese adicional imposta neste trabalho de que $\hat{\omega}$ seja não-degenerada pode facilmente ser eliminada substituindo $V$ e $L$ pelos espaços quociente $V / \operatorname{ker} \hat{\omega}$ e $L / \operatorname{ker} \hat{\omega}$.

No caso de formas simpléticas $(\operatorname{dim} \hat{T}=1, k=1$, ker $\hat{\omega}=\{0\})$, onde o conceito de um subespaço polilagrangeano se reduz ao conceito familiar de um subespaço lagrangeano, é bem conhecido que não vale tal unicidade. A maior utilidade dos subespaços polilagrangeanos, que generalizam os subespaços lagrangeanos do caso simplético para formas a valores vetoriais, é que são essenciais para a obtenção de um teorema de Darboux.

O caso de formas comuns não-degeneradas de grau $>2(\operatorname{dim} \hat{T}=1, k>1, \operatorname{ker} \hat{\omega}=\{0\})$ tem sido estudado na literatura $[3,19]$ sob o rótulo "formas multissimpléticas", porém sem destaque para o papel central do conceito de subespaço polilagrangeano, que está apenas implícito através do critério de dimensão formulado no Teorema 1.3, usado como definição. De qualquer forma, a restrição imposta pela existência de um subespaço polilagrangeano é necessária para a obtenção de uma "base de Darboux" para a forma $\hat{\omega}$.

\subsection{Formas parcialmente horizontais em espaços vetoriais}

Nesta seção apresentamos os conceitos análogos aos introduzidos na seção anterior para o caso de formas comuns que estão sujeitas à restrição de serem parcialmente horizontais em relação a algum subespaço "vertical" dado, o que requer algumas modificações. Novamente, introduzimos um tipo especial de subespaço maximal isotrópico, ou lagrangeano, chamado de subespaço multilagrangeano, que desempenha um papel fundamental na teoria das formas multissimpléticas, a ser desenvolvida no próximo capítulo. 
Sejam $W$ um espaço vetorial de dimensão finita e $V$ um subespaço fixo de $W$, o espaço vertical. Como no início do capítulo, denotamos o espaço quociente $W / V$, o espaço base, por $T$, pondo $\operatorname{dim} T=n$, e a projeção canônica de $W$ em $T$ por $\pi$, obtendo assim a sequência exata (1.3). Ademais, suponha que $\omega$ é uma $(k+1)$-forma em $W$ que é $(k+1-r)$-horizontal, onde $1 \leqslant r \leqslant k$, e $r \geqslant k+1-n$ caso $k>n:^{5}$

$$
\omega \in \bigwedge_{r}^{k+1} W^{*} .
$$

Tratando-se de uma caso especial da situação estudada anteriormente, as definições iniciais da primeira seção se aplicam sem alterações: isso inclui os conceitos do núcleo e do suporte, a noção de uma forma não-degenerada, a idéia do posto e a definição do complemento $\ell$-ortogonal de um subespaço. A primeira diferença importante ocorre no estudo dos subespaços isotrópicos, pois no caso de formas parcialmente horizontais, consideraremos apenas subespaços isotrópicos $L$ do espaço total $W$ contidos no espaço vertical $V$, o que implica em um conceito de maximalidade diferente do anterior: um subespaço $L$ de $V$ será chamado maximal isotrópico em $V$ (relativo a $\omega$ ) se $L$ é isotrópico (relativo a $\omega$ ) e se para qualquer outro subespaço $L^{\prime}$ de $V$ que também é isotrópico (relativo a $\omega$ ), vale

$$
L \subset L^{\prime} \Longrightarrow L=L^{\prime}
$$

Usando a restrição da aplicação linear

$$
\begin{aligned}
\omega_{W}^{b}: W & \longrightarrow \bigwedge^{k} W^{*} \\
w & \longmapsto \mathrm{i}_{w} \omega
\end{aligned},
$$

definida conforme a equação (1.38), ao subespaço vertical $V$, que é uma aplicação linear

$$
\begin{array}{cccc}
\omega_{V}^{b}: V & \longrightarrow & \bigwedge_{r-1}^{k} W^{*} \\
v & \longmapsto & \mathrm{i}_{v} \omega
\end{array},
$$

e definindo, para qualquer subespaço $L$ de $V$,

$$
\bigwedge_{r-1}^{k} L^{\perp}=\bigwedge^{k} L^{\perp} \cap \bigwedge_{r-1}^{k} W^{*}
$$

podemos concluir que um subespaço $L$ de $V$ é isotrópico se e somente se vale a inclusão

$$
\omega_{V}^{b}(L) \subset \bigwedge_{r-1}^{k} L^{\perp}
$$

que podemos, de modo trivial, reescrever na forma

$$
\omega_{V}^{b}(L) \subset \omega_{V}^{b}(V) \cap \bigwedge_{r-1}^{k} L^{\perp} .
$$

\footnotetext{
${ }^{5}$ Excluiremos os casos triviais $k=0$ e $k=r+1$ pois estes se enquadram no formalismo desenvolvido na seção anterior, porém com $\operatorname{dim} \hat{T}=1$.
} 
Também podemos concluir que um subespaço $L$ de $V$ que contém $\operatorname{ker} \omega_{V}^{b}=V \cap \operatorname{ker} \omega$ é maximal isotrópico em $V$ se e somente se vale a igualdade

$$
\omega_{V}^{b}(L)=\omega_{V}^{b}(V) \cap \bigwedge_{r-1}^{k} L^{\perp} .
$$

De fato, para qualquer subespaço $L$ de $V$, vale a identidade

$$
\omega_{V}^{b}\left(L^{\omega, 1} \cap V\right)=\omega_{V}^{b}(V) \cap \bigwedge_{r-1}^{k} L^{\perp}
$$

pois ambos os lados são iguais ao subespaço de $\bigwedge_{r-1}^{k} W^{*}$ formado pelos elementos da forma $\mathrm{i}_{v} \omega$ com $v \in V$ e tais que $\mathrm{i}_{u} \mathrm{i}_{v} \omega=0$ para todo $u \in L$.

Posto isso, podemos introduzir um conceito que constitui a chave mestre para o estudo de formas multissimpléticas: a existência de subespaços isotrópicos maximais "especiais", que nós chamaremos de subespaços multilagrangeanos:

Definição 1.2 Sejam $W$ um espaço vetorial de dimensão finita e $V$ um subespaço fixo de $W$, com $n=\operatorname{dim}(W / V)$, e seja $\omega$ uma $(k+1)$-forma em $W$. Dizemos que $\omega$ é uma forma multilagrangeana de posto $N$ e grau de horizontalidade $k+1-r$, onde $1 \leqslant r \leqslant k e$ $k+1-r \leqslant n$, se $\omega$ é $(k+1-r)$-horizontal e se $V$ admite um subespaço $L$ de codimensão $N$ que é multilagrangeano, i.e., que satisfaz

$$
\omega_{V}^{b}(L)=\bigwedge_{r-1}^{k} L^{\perp}
$$

Quando $k=n$ e $r=2$ e $\omega$ for não-degenerada, dizemos que $\omega$ é uma forma multissimplética.

O seguinte teorema mostra que um subespaço multilagrangeano, quando existir, contém o núcleo de $\omega$ e portanto realmente é um tipo especial de subespaço maximal isotrópico em $V$.

Teorema 1.5 Sejam $W$ um espaço vetorial de dimensão finita e $V$ um subespaço fixo de $W$, com $n=\operatorname{dim}(W / V)$, e seja $\omega$ uma $(k+1)$-forma multilagrangeana em $W$ de posto $N$ e grau de horizontalidade $k+1-r$, onde $1 \leqslant r \leqslant k$ e $k+1-r \leqslant n$, com subespaço multilagrangeano $L$ de $V$. Então vale $N+n \geqslant k$ (exceto se $\omega \equiv 0$ ) e $L$ contém o núcleo de $\omega$ :

$$
\operatorname{ker} \omega \subset L \text {. }
$$

A dimensão de L é dada por

$$
\operatorname{dim} L=\operatorname{dim} \operatorname{ker} \omega+\sum_{s=0}^{r-1}\left(\begin{array}{c}
N \\
s
\end{array}\right)\left(\begin{array}{c}
n \\
k-s
\end{array}\right)
$$

onde é subentendido que, por definição, $\left(\begin{array}{l}p \\ q\end{array}\right)=0$ se $q>p$. 
DemonstraÇÃo. Primeiro, observamos que se $N+n<k$, temos $\bigwedge^{k} L^{\perp}=\{0\}$ e portanto os dois lados da equação (1.59) se anulam; assim, $L$ é contido em ker $\omega$ e portanto ker $\omega$ tem codimensão $<k$ em $W$, o que força $\omega \equiv 0$ pois a $(k+1)$-forma no espaço quociente $W / \operatorname{ker} \omega$ induzida por $\omega$ se anula. (Este argumento mostra, mais geralmente, que para qualquer $(k+1)$-forma em $W$ que é $(k+1-r)$-horizontal, $V$ não pode conter subespaços isotrópicos de codimensão $<k-n$.) Supondo então que $\operatorname{dim} L^{\perp}=N+n \geqslant k$ e usando que $\operatorname{dim} V^{\perp}=n$ e $V^{\perp} \subset L^{\perp}$, podemos para todo $w \in W \backslash L$ encontrar um conjunto linearmente independente de 1-formas $w_{1}^{*}, \ldots, w_{k}^{*} \in L^{\perp}$ tal que $w_{r}^{*}, \ldots, w_{k}^{*} \in V^{\perp}$ e $\left\langle w_{1}^{*}, w\right\rangle=1 \mathrm{e}\left\langle v_{i}^{*}, v\right\rangle=0$ para $i>1$. Conforme a definição de subespaço multilagrangeano, existe $u \in L$ satisfazendo

$$
\mathrm{i}_{u} \omega=w_{1}^{*} \wedge \ldots \wedge w_{k}^{*} \Rightarrow \mathrm{i}_{w} \mathrm{i}_{u} \omega=w_{2}^{*} \wedge \ldots \wedge w_{k}^{*} \neq 0
$$

e portanto $w \notin \operatorname{ker} \omega$. Logo segue que $\operatorname{ker} \omega \subset L$. Assim vale

$$
\operatorname{dim} L-\operatorname{dim} \operatorname{ker} \omega=\operatorname{dim} \omega_{V}^{b}(L)=\operatorname{dim} \bigwedge_{r-1}^{k} L^{\perp} .
$$

Para calcular esta dimensão, procedemos como no início do capítulo, introduzindo uma base $\left\{e_{1}^{L}, \ldots, e_{l}^{L}, e_{1}^{L^{\prime}}, \ldots, e_{N}^{L^{\prime}}, e_{1}^{T}, \ldots, e_{n}^{T}\right\}$ de $W$ tal que os primeiros $l$ vetores formam uma base de $L$, os seguintes $N$ vetores formam uma base de um subespaço $L^{\prime}$ complementar a $L$ em $V$ e os últimos $n$ vetores formam uma base de um subespaço $H$ complementar a $V$ em $W$, isomorfo a $T$. Então em termos da base dual $\left\{e_{L}^{1}, \ldots, e_{L}^{l}, e_{L^{\prime}}^{1}, \ldots, e_{L^{\prime}}^{N}, e_{T}^{1}, \ldots, e_{T}^{n}\right\}$ de $W^{*}$, concluímos que $\left\{e_{L^{\prime}}^{i_{1}} \wedge \ldots \wedge e_{L^{\prime}}^{i_{s}} \wedge e_{T}^{\mu_{1}} \wedge \ldots \wedge e_{T}^{\mu_{k-s}} \mid 0 \leqslant s \leqslant r-1,1 \leqslant i_{1}<\ldots<i_{s} \leqslant N\right.$, $\left.1 \leqslant \mu_{1}<\ldots<\mu_{k-s} \leqslant n\right\}$ é uma base de $\bigwedge_{r-1}^{k} L^{\perp}$, provando a fórmula (1.61).

A existência de um subespaço multilagrangeano pode ser detectada por um simples critério de dimensão:

Teorema 1.6 Sejam $W$ um espaço vetorial de dimensão finita e $V$ um subespaço fixo de $W$, com $n=\operatorname{dim}(W / V)$, e seja $\omega$ uma $(k+1)$-forma em $W$ que é $(k+1-r)$-horizontal, onde $1 \leqslant r \leqslant k$ e $k+1-r \leqslant n$. Se $L$ é um subespaço isotrópico de $V$ de codimensão $N$ contendo ker $\omega$ e tal que

$$
\operatorname{dim} L=\operatorname{dim} \operatorname{ker} \omega+\sum_{s=0}^{r-1}\left(\begin{array}{c}
N \\
s
\end{array}\right)\left(\begin{array}{c}
n \\
k-s
\end{array}\right),
$$

onde é subentendido que, por definição, $\left(\begin{array}{l}p \\ q\end{array}\right)=0$ se $q>p$, então L é um subespaço multilagrangeano de $V$ e $\omega$ é uma forma multilagrangeana.

Demonstração. Sendo $L$ um subespaço isotrópico de $V$ contendo ker $\omega$, a restrição da aplicação linear $\omega_{V}^{b}$ (veja a equação (1.53)) a $L$ leva $L$ em $\bigwedge_{r-1}^{k} L^{\perp}$ e induz uma aplicação linear injetora do espaço quociente $L / \operatorname{ker} \omega$ em $\bigwedge_{r-1}^{k} L^{\perp}$ que, segundo a condição (1.62), é um isomorfismo linear; logo, vale a igualdade (1.59). 
Um caso particularmente simples ocorre para $r=1$, pois toda $(k+1)$-forma que é $k$-horizontal e multilagrangeana tem posto 0 e subespaço multilagrangeano $V$. (De fato, a condição de que $\omega$ seja $k$-horizontal é equivalente à condição de que $V$ seja isotrópico e obviamente, neste caso, $V$ satisfaz os demais critérios do Teorema 1.6.)

\subsection{O símbolo}

Nesta seção, a nossa meta é exibir uma relação simples e geral entre formas multilagrangeanas e formas polilagrangeanas: o símbolo de uma forma multilagrangeana é uma forma polilagrangeana e, em particular, o símbolo de uma forma multissimplética é uma forma polissimplética, a valores em um espaço de formas no espaço base.

Para tanto, suporemos como na seção anterior que $W$ é um espaço vetorial de dimensão finita e $V$ é um subespaço fixo de $W$, o espaço vertical, e denotaremos o espaço quociente $W / V$, o espaço base, por $T$, pondo $\operatorname{dim} T=n$, e a projeção canônica de $W$ em $T$ por $\pi$, obtendo assim a sequência exata (1.3). Também suporemos como na seção anterior que $\omega$ é uma $(k+1)$-forma em $W$ que é $(k+1-r)$-horizontal, onde $1 \leqslant r \leqslant k$, e $r \geqslant k+1-n$ caso $k>n$ :

$$
\omega \in \bigwedge_{r}^{k+1} W^{*} .
$$

Então o símbolo $\hat{\omega}$ de $\omega$, definido como no início do capítulo, será uma $r$-forma em $V$ a valores no espaço $\bigwedge^{k+1-r} T^{*}$ :

$$
\hat{\omega} \in \bigwedge^{r} V^{*} \otimes \bigwedge^{k+1-r} T^{*} .
$$

Usando o isomorfismo canônico $\bigwedge^{k+1-r} T^{*} \cong \bigwedge_{0}^{k+1-r} W^{*}$ como identificação, temos

$$
\hat{\omega}\left(v_{1}, \ldots, v_{r}\right)=\mathrm{i}_{v_{r}} \ldots \mathrm{i}_{v_{1}} \omega \quad \text { para } v_{1}, \ldots, v_{r} \in V .
$$

Mais explicitamente, usando uma cisão $\mathfrak{s}$ de $\pi$, temos

$$
\begin{gathered}
\hat{\omega}\left(v_{1}, \ldots, v_{r}\right)\left(t_{1}, \ldots, t_{k+1-r}\right)=\omega\left(v_{1}, \ldots, v_{r}, \mathfrak{s}\left(t_{1}\right), \ldots, \mathfrak{s}\left(t_{k+1-r}\right)\right) \\
\text { para } v_{1}, \ldots, v_{r} \in V, t_{1}, \ldots, t_{k+1-r} \in T,
\end{gathered}
$$

conforme já foi mostrado no início de capítulo.

Uma análise direta da fórmula (1.66) mostra que um subespaço $L$ do espaço vertical $V$ é isotrópico relativo a $\hat{\omega}$ se é isotrópico relativo a $\omega$ (a recíproca não é verdadeira). Além disso, temos 
Teorema 1.7 Sejam $W$ um espaço vetorial de dimensão finita e $V$ um subespaço fixo de $W$, com $n=\operatorname{dim}(W / V)$, seja $\omega$ uma $(k+1)$-forma em $W$ que é $(k+1-r)$-horizontal, onde $1 \leqslant r \leqslant k$ e $k+1-r \leqslant n$, e seja $\hat{\omega}$ o símbolo de $\omega$, que é uma $r$-forma em $V$ a valores no espaço $\bigwedge^{k+1-r} T^{*}$. Suponha que $\omega$ é multilagrangeana, com subespaço multilagrangeano $L$ de $V$. Então $\hat{\omega}$ é polilagrangeana, com subespaço polilagrangeano L. Se $\omega$ for multissimplética então $\hat{\omega}$ será polissimplética.

DemonstraçÃo. Escolhendo um subespaço horizontal $H$ de $W$ qualquer e utilizando as decomposições diretas (1.17) e (1.18), com $H^{\perp} \cong V^{*}$, observamos que para mostrar que $L$ é polilagrangeano relativo a $\hat{\omega}$, precisamos estabelecer a igualdade

$$
\hat{\omega}^{b}(L) \cong \bigwedge^{r-1}\left(L^{\perp} \cap H^{\perp}\right) \otimes \bigwedge^{k+1-r} T^{*} .
$$

Para tanto, fazemos uso do isomorfismo canônico $\bigwedge^{k+1-r} T^{*} \cong \bigwedge^{k+1-r} W^{*}=\bigwedge^{k+1-r} V^{\perp}$ e da inclusão $V^{\perp} \subset L^{\perp}$, assim como do fato de que o espaço $\bigwedge^{r-1}\left(L^{\perp} \cap H^{\perp}\right) \otimes \bigwedge^{k+1-r} V^{\perp}$ é gerado por elementos que podem ser escritas na forma

$$
\hat{\alpha}=\left(w_{1}^{*} \wedge \ldots \wedge w_{r-1}^{*}\right) \otimes\left(w_{r}^{*} \wedge \ldots \wedge w_{k}^{*}\right) \quad \text { onde } \quad \alpha=w_{1}^{*} \wedge \ldots \wedge w_{k}^{*}
$$

com $w_{1}^{*}, \ldots, w_{r-1}^{*} \in L^{\perp} \cap H^{\perp}$ e $w_{r}^{*}, \ldots, w_{k}^{*} \in V^{\perp} \subset L^{\perp}$. Como $L$ é multilagrangeano relativo a $\omega$, existe $u \in L$ tal que $\alpha=\mathrm{i}_{u} \omega$ e portanto $\hat{\alpha}=\mathrm{i}_{u} \hat{\omega}$, mostrando que $L$ é polilagrangeano relativo a $\hat{\omega}$.

Mostramos que o espaço multilagrangeano de uma forma multilagrangeana $\omega$ é o mesmo que o espaço polilagrangeano do seu símbolo $\hat{\omega}$, mas é importante ressaltar que, em geral, o núcleo de $\omega$ não é o mesmo que o núcleo de $\hat{\omega}$ : podemos apenas afirmar que

$$
\operatorname{ker} \omega \subset \operatorname{ker} \hat{\omega} \text {. }
$$

Em particular, $\omega$ pode ser não-degenerada sem que $\hat{\omega}$ o seja. Além disso, consideraremos as projeções $\hat{\omega}_{t_{1}, \ldots, t_{k+1-r}}$ de $\hat{\omega}$ ao longo dos tensores decomponíveis $t_{1} \wedge \ldots \wedge t_{k+1-r} \in \bigwedge^{k+1-r} T$ : explicitamente, usando uma cisão $\mathfrak{s}$ de $\pi$, temos para os respectivos núcleos

$$
\operatorname{ker} \hat{\omega}_{t_{1}, \ldots, t_{k+1-r}}=\left\{v \in V \mid \begin{array}{c}
\omega\left(v, v_{1}, \ldots, v_{r-1}, \mathfrak{s}\left(t_{1}\right), \ldots, \mathfrak{s}\left(t_{k+1-r}\right)\right)=0 \\
\operatorname{para} v_{1}, \ldots, v_{r-1} \in V, t_{1}, \ldots, t_{k+1-r} \in T
\end{array}\right\},
$$

sendo que conforme os resultados obtidos na primeira seção, ker $\hat{\omega}$ é a intersecção e, para $0<k+1-r<n, L$ é a soma de todos estes núcleos parciais.

\subsection{Exemplos canônicos}

Nesta seção introduziremos o que chamaremos de exemplo canônico de um espaço vetorial polilagrangeano, na primeira subseção, e de um espaço vetorial multilagrangeano, na segunda 
subseção. Como veremos nas seções seguintes, toda forma polilagrangeana não degenerada e toda forma multilagrangeana não degenerada é equivalente à forma canônica aqui apresentada, ou seja, existe um isomorfismo linear entre os respectivos espaços vetoriais que relaciona as duas. Desde já fixaremos o inteiro $k$ que caracteriza o grau da forma (que é $k+1$ ), com $k \geqslant 1$, e no caso multilagrangeano, o inteiro $r$ que caracteriza o grau de horizontalidade da forma (que é $k-r$ ), com $1 \leqslant r \leqslant k{ }^{6}$ Assim, evitamos sobrecarregar a notação com prefixos do tipo " $k-$ " ou " $(k, r)-$ ".

\subsubsection{A forma polilagrangeana canônica}

Sejam $E$ um espaço vetorial de dimensão $N$ e $\hat{T}$ um espaço vetorial de dimensão $\hat{n}$. Defina

$$
V_{0}=E \oplus\left(\left(\bigwedge^{k} E^{*}\right) \otimes \hat{T}\right) .
$$

Definição 1.3 Com $V_{0}$ como definido acima, a forma polilagrangeana canônica de posto $N$ é a $(k+1)$-forma não degenerada em $V_{0}$ a valores em $\hat{T}$ dada por

$$
\hat{\omega}_{0}\left(\left(u_{0}, \alpha_{0} \otimes \hat{t}_{0}\right), \ldots,\left(u_{k}, \alpha_{k} \otimes \hat{t}_{k}\right)\right)=\sum_{i=0}^{k}(-1)^{i} \alpha_{i}\left(u_{0}, \ldots, \widehat{u}_{i}, \ldots, u_{k}\right) \hat{t}_{i} .
$$

Se $k=1$ chamaremos $\hat{\omega}_{0}$ de forma polissimplética canônica.

Cabe observar que para o caso $\hat{T}=\mathbb{R}$ esta construção já se encontra em [19] e [3].

Para provar a afirmação contida nesta definição, notamos que $\hat{\omega}_{0}$ é de fato não degenerada e que, considerando $E \mathrm{e}$

$$
L=\left(\bigwedge^{k} E^{*}\right) \otimes \hat{T}
$$

como subespaços de $V_{0}$, vale $V_{0}=L \oplus E$ onde

$$
L \text { é polilagrangeano e } E \text { é } k \text {-isotrópico , }
$$

pois $L$ é obviamente 1-isotrópico e tem a dimensão requerida pelo Teorema 1.3:

$$
\operatorname{dim} L=\hat{n}\left(\begin{array}{c}
N \\
k
\end{array}\right) \quad \text { onde } \operatorname{codim}_{V_{0}} L=N .
$$

Em termos de bases, seja $\left\{\hat{e}_{a} \mid 1 \leqslant a \leqslant \hat{n}\right\}$ uma base qualquer de $\hat{T}$ com base dual $\left\{\hat{e}^{a} \mid 1 \leqslant a \leqslant \hat{n}\right\}$ de $\hat{T}^{*}$ e $\left\{e_{i} \mid 1 \leqslant i \leqslant N\right\}$ uma base qualquer de $E$ com base dual $\left\{e^{i} \mid 1 \leqslant i \leqslant N\right\}$ de $E^{*}$. Para $1 \leqslant a \leqslant \hat{n}$ e $1 \leqslant i_{1}<\ldots<i_{k} \leqslant N$, defina

$$
e_{a}^{i_{1} \ldots i_{k}}=e^{i_{1}} \wedge \ldots \wedge e^{i_{k}} \otimes \hat{e}_{a} \quad, \quad e_{i_{1} \ldots i_{k}}^{a}=e_{i_{1}} \wedge \ldots \wedge e_{i_{k}} \otimes \hat{e}^{a} .
$$

\footnotetext{
${ }^{6}$ Estamos excluindo os caso extremos $r=0$ e $r=k+1$, que são acomodados no caso polilagrangeano, com $\hat{T}=\mathbb{R}$.
} 
Assim obtemos uma base $\left\{e_{i}, e_{a}^{i_{1} \ldots i_{k}} \mid 1 \leqslant a \leqslant \hat{n}, 1 \leqslant i \leqslant N, 1 \leqslant i_{1}<\ldots<i_{k} \leqslant N\right\}$ de $V_{0}$ com base dual $\left\{e^{i}, e_{i_{1} \ldots i_{k}}^{a} \mid 1 \leqslant a \leqslant \hat{n}, 1 \leqslant i \leqslant N, 1 \leqslant i_{1}<\ldots<i_{k} \leqslant N\right\}$ de $V_{0}^{*}$, que chamaremos de bases de Darboux, tal que

$$
\hat{\omega}_{0}=\frac{1}{k !}\left(e_{i_{1} \ldots i_{k}}^{a} \wedge e^{i_{1}} \wedge \ldots \wedge e^{i_{k}}\right) \otimes \hat{e}_{a} .
$$

$\mathrm{Na}$ Seção 1.5 veremos que qualquer espaço polilagrangeano admite uma decomposição na soma direta de um (do) subespaço polilagrangeano e uma componente $k$-isotrópica. É justamente isso que faz com que toda forma polilagrangeana seja equivalente à canônica, o que é o conteúdo do teorema de Darboux para espaços vetoriais polilagrangeanos.

\subsubsection{A forma multilagrangeana canônica}

Sejam $F$ um espaço vetorial de dimensão $N+n$ e $E$ um subespaço fixo de $F$ de dimensão $N$. Denotando o espaço quociente $F / E$ por $T$, de modo que $\operatorname{dim} T=n$, e a projeção canônica de $F$ em $T$ por $\rho$, obtemos a seguinte sequência exata de espaços vetoriais:

$$
0 \longrightarrow E \longrightarrow F \stackrel{\rho}{\longrightarrow} T \longrightarrow 0 \text {. }
$$

Defina

$$
W_{0}=F \oplus \bigwedge_{r-1}^{k} F^{*} \quad, \quad V_{0}=E \oplus \bigwedge_{r-1}^{k} F^{*} \quad, \quad \pi_{0}=\rho \circ p_{1},
$$

onde $\operatorname{pr}_{1}: W_{0} \rightarrow F$ é a projeção canônica, o que leva à seguinte sequência exata de espaços vetoriais:

$$
0 \longrightarrow V_{0} \longrightarrow W_{0} \stackrel{\pi_{0}}{\longrightarrow} T \longrightarrow 0 .
$$

Definição 1.4 Com $W_{0}$ e $V_{0}$ como definidos acima, a forma multilagrangeana canônica de posto $N$ e grau de horizontalidade $k+1-r$ é a $(k+1)$-forma $(k+1-r)$ horizontal em $W_{0}$ dada por

$$
\omega_{0}\left(\left(u_{0}, \alpha_{0}\right), \ldots,\left(u_{k}, \alpha_{k}\right)\right)=\sum_{i=0}^{k}(-1)^{i} \alpha_{i}\left(u_{0}, \ldots, \widehat{u}_{i}, \ldots, u_{k}\right) .
$$

Se $k=n$ e $r=2$ chamaremos $\omega_{0}$ de forma multissimplética canônica.

Note que quando $r=1$, a forma $\omega_{0}$ é degenerada, com núcleo

$$
\text { ker } \omega_{0}=E \quad \text { se } r=1,
$$

enquanto que quando $r>1$, a forma $\omega_{0}$ é não degenerada. De fato, seja $(u, \alpha) \in W_{0} \backslash\{0\}$. Se $\alpha \neq 0$ então existem vetores $u_{1}, \ldots, u_{k} \in F$ tais que

$$
\omega_{0}\left((u, \alpha),\left(u_{1}, 0\right), \ldots,\left(u_{k}, 0\right)\right)=\alpha\left(u_{1}, \ldots, u_{k}\right) \neq 0,
$$


e portanto $(u, \alpha) \notin \operatorname{ker} \omega_{0}$. Se $r=1$ e $u \notin E$ ou se $r>1$ e $u \neq 0$ existe uma $k$-forma $(k+1-r)$-horizontal $\beta \in \bigwedge_{r-1}^{k} F^{*}$ tal que $\mathrm{i}_{u} \beta \neq 0$; logo, existem vetores $u_{2}, \ldots, u_{k} \in F$ tais que

$$
\omega_{0}\left((u, 0),(0, \beta),\left(u_{2}, 0\right), \ldots,\left(u_{k}, 0\right)\right)=-\beta\left(u, u_{2}, \ldots, u_{k}\right) \neq 0,
$$

e portanto $(u, 0) \notin \operatorname{ker} \omega_{0}$.

No que segue, suporemos que $N>0$, pois se tomarmos $E=\{0\}$, estamos reduzidos ao caso polilagrangeano em $T \oplus \bigwedge^{k} T^{*}$, com $\hat{T}=\mathbb{R}$, já estudado em [19] e [3]. Do mesmo modo, suporemos que $r>1$, pois quando $r=1$, segue $\bigwedge_{0}^{k} F^{*} \cong \bigwedge^{k} T^{*}$ e logo após passagem ao quociente módulo o núcleo de $\omega_{0}$ estamos mais uma vez reduzidos ao caso polilagrangeano em $T \oplus \bigwedge^{k} T^{*}$, com $\hat{T}=\mathbb{R}$, já estudado em [19] e [3].

Para provar a afirmação contida na definição acima (com as hipóteses adicionais de que $N>0$ e $r>1)$, notamos que $\omega_{0}$ é de fato $(k+1-r)$-horizontal e que, considerando $F$ e

$$
L=\bigwedge_{r-1}^{k} F^{*}
$$

como subespaços de $W_{0}$, vale $W_{0}=L \oplus F$ e $V_{0}=L \oplus E$ onde

$$
L \text { é multilagrangeano , } F \text { é } k \text {-isotrópico } \quad, \quad E \text { é }(r-1) \text {-isotrópico , }
$$

pois $L$ é obviamente 1-isotrópico e tem a dimensão requerida pelo Teorema 1.6:

$$
\operatorname{dim} L=\sum_{s=0}^{r-1}\left(\begin{array}{c}
N \\
s
\end{array}\right)\left(\begin{array}{c}
n \\
k-s
\end{array}\right) \text { onde } \operatorname{codim}_{V_{0}} L=N
$$

Em termos de bases, seja $\left\{e_{i}, e_{\mu} \mid 1 \leqslant i \leqslant N, 1 \leqslant \mu \leqslant n\right\}$ uma base de $F$ com base dual $\left\{e^{i}, e^{\mu} \mid 1 \leqslant i \leqslant N, 1 \leqslant \mu \leqslant n\right\}$ de $F^{*}$ tal que $\left\{e_{i} \mid 1 \leqslant i \leqslant N\right\}$ é uma base de $E$ e $\left\{e_{\mu} \mid 1 \leqslant \mu \leqslant n\right\}$ é uma base de um subespaço $H$ de $F$ complementar a $E$, isomorfo a $T$. Para $0 \leqslant s \leqslant r, 1 \leqslant i_{1}<\ldots<i_{s} \leqslant N$ e $1 \leqslant \mu_{1}<\ldots<\mu_{k-s} \leqslant N$, defina

$$
\begin{aligned}
& e_{i_{1} \ldots i_{s} ; \mu_{1} \ldots \mu_{k-s}}=e_{i_{1}} \wedge \ldots \wedge e_{i_{s}} \wedge e_{\mu_{1}} \wedge \ldots \wedge e_{\mu_{k-s}} \\
& e^{i_{1} \ldots i_{s} ; \mu_{1} \ldots \mu_{k-s}}=e^{i_{1}} \wedge \ldots \wedge e^{i_{s}} \wedge e^{\mu_{1}} \wedge \ldots \wedge e^{\mu_{k-s}}
\end{aligned} .
$$

Assim obtemos uma base

$$
\left\{e_{i}, e_{\mu}, e^{i_{1} \ldots i_{s} ; \mu_{1} \ldots \mu_{k-s}} \mid 0 \leqslant s \leqslant r-1, \begin{array}{c}
1 \leqslant i \leqslant N, 1 \leqslant i_{1}<\ldots<i_{s} \leqslant N \\
1 \leqslant \mu \leqslant n, 1 \leqslant \mu_{1}<\ldots<\mu_{k-s} \leqslant n
\end{array}\right\}
$$

de $W_{0}$ com base dual

$$
\left\{e^{i}, e^{\mu}, e_{i_{1} \ldots i_{s} ; \mu_{1} \ldots \mu_{k-s}} \mid 0 \leqslant s \leqslant r-1, \begin{array}{c}
1 \leqslant i \leqslant N, 1 \leqslant i_{1}<\ldots<i_{s} \leqslant N \\
1 \leqslant \mu \leqslant n, 1 \leqslant \mu_{1}<\ldots<\mu_{k-s} \leqslant n
\end{array}\right\}
$$


de $W_{0}^{*}$, que chamaremos de bases de Darboux, tal que

$$
\omega_{0}=\sum_{s=0}^{r-1} \frac{1}{s !} \frac{1}{(k-s) !} e_{i_{1} \ldots i_{s} ; \mu_{1} \ldots \mu_{k-s}} \wedge e^{i_{1}} \wedge \ldots \wedge e^{i_{s}} \wedge e^{\mu_{1}} \wedge \ldots \wedge e^{\mu_{k-s}}
$$

Assim, temos para o símbolo

$$
\hat{\omega}_{0}=\frac{1}{(r-1) !} \frac{1}{(k+1-r) !}\left(e_{i_{1} \ldots i_{r-1} ; \mu_{1} \ldots \mu_{k+1-r}} \wedge e^{i_{1}} \wedge \ldots \wedge e^{i_{r-1}}\right) \otimes\left(e^{\mu_{1}} \wedge \ldots \wedge e^{\mu_{k+1-r}}\right) .
$$

Na Seção 1.6 veremos que qualquer espaço multilagrangeano admite uma decomposição na soma direta de um (do) subespaço multilagrangeano e uma componente $k$-isotrópica que, por sua vez, contém uma componente $(r-1)$-isotrópica tal que o quociente destas duas componentes é isomorfo ao espaço base $T$. É justamente isto que faz com que toda forma multilagrangeana seja equivalente à canônica, o que é o conteúdo do teorema de Darboux para espaços vetoriais multilagrangeanos.

\subsection{Espaços vetoriais polilagrangeanos}

Nesta seção, mostraremos que toda forma polilagrangeana admite uma base de Darboux, ou base canônica, na qual é representada por uma fórmula do tipo dada na equação (1.74) da Seção 1.4 .

Definição 1.5 Sejam $V$ e $\hat{T}$ espaços vetoriais de dimensão finita, com $\hat{n} \equiv \operatorname{dim} \hat{T}$, e seja $\hat{\omega}$ uma $(k+1)$-forma em $V$ a valores em $\hat{T}$ que é polilagrangeana de posto $N$, com subespaço polilagrangeano L. Uma base de Darboux polilagrangeana ou base canônica polilagrangeana é uma base $\left\{e_{i}, e_{a}^{i_{1} \ldots i_{k}} \mid 1 \leqslant a \leqslant \hat{n}, 1 \leqslant i \leqslant N, 1 \leqslant i_{1}<\ldots<i_{k} \leqslant N\right\}$ de um subespaço de $V$ complementar a ker $\hat{\omega}$, com base dual $\left\{e^{i}, e_{i_{1} \ldots i_{k}}^{a} \mid 1 \leqslant a \leqslant \hat{n}\right.$, $\left.1 \leqslant i \leqslant N, 1 \leqslant i_{1}<\ldots<i_{k} \leqslant N\right\}$ do subespaço supp $\hat{\omega}=(\operatorname{ker} \hat{\omega})^{\perp} d e V^{*}$, em conjunto com uma base $\left\{\hat{e}_{a} \mid 1 \leqslant a \leqslant \hat{n}\right\}$ de $\hat{T}$, com base dual $\left\{\hat{e}^{a} \mid 1 \leqslant a \leqslant \hat{n}\right\}$ de $\hat{T}^{*}$, tal que

$$
\hat{\omega}=\frac{1}{k !}\left(e_{i_{1} \ldots i_{k}}^{a} \wedge e^{i_{1}} \wedge \ldots \wedge e^{i_{k}}\right) \otimes \hat{e}_{a} .
$$

Se $k=1$ chamaremos tal base de base de Darboux polissimplética ou base canônica polissimplética.

A existência de uma base canônica para toda forma polilagrangeana decorre da existência de uma decomposição direta $V=L \oplus E$ de $V$ do tipo apresentado para $V_{0}$ na Seção 1.4. 
Teorema 1.8 Sejam $V$ e $\hat{T}$ espaços vetoriais de dimensão finita, com $\hat{n} \equiv \operatorname{dim} \hat{T}$, e seja $\hat{\omega}$ uma $(k+1)$-forma em $V$ a valores em $\hat{T}$ que é polilagrangeana de posto $N$, com subespaço polilagrangeano L. Então existe um subespaço $k$-isotrópico $E$ de $V$ complementar a L, i.e., tal que

$$
V=E \oplus L
$$

Demonstração. Seja $E_{0}$ um subespaço de $V$ de dimensão $N^{\prime}$ que é $k$-isotrópico e tal que $E_{0} \cap L=\{0\}$. (Por exemplo, se $N^{\prime} \leqslant k, E_{0}$ pode ser qualquer subespaço de $V$ tal que $E_{0} \cap L=\{0\}$.) Se $N^{\prime}=N$ o teorema já está demonstrado. Caso contrário, escolha uma base $\left\{e_{1}, \ldots, e_{N}\right\}$ de um subespaço de $V$ complementar a $L$ tal que os primeiros $N^{\prime}$ vetores formam uma base de $E_{0}$, e denote a correspondente base dual de $L^{\perp}$ por $\left\{e^{1}, \ldots, e^{N}\right\}$. Provaremos que existe um vetor $u \in V \backslash\left(E_{0} \oplus L\right)$ tal que o subespaço $E_{1}$ de $V$ gerado por $u$ e $E_{0}$ é $k$-isotrópico e tal que $E_{1} \cap L=\{0\}$, e como $\operatorname{dim} E_{1}=N^{\prime}+1$, a afirmação do teorema segue por indução. Para tanto, consideramos uma base qualquer $\left\{\hat{e}_{a} \mid 1 \leqslant a \leqslant \hat{n}\right\}$ de $\hat{T}$ com base dual $\left\{\hat{e}^{a} \mid 1 \leqslant a \leqslant \hat{n}\right\}$ de $\hat{T}^{*}$ e, escolhendo um subespaço $L^{\prime}$ qualquer de $L$ complementar a ker $\hat{\omega}$, podemos usar o fato de $L$ ser polilagrangeano para concluir que existe uma única base $\left\{e_{a}^{i_{1} \ldots i_{k}} \mid 1 \leqslant a \leqslant \hat{n}, 1 \leqslant i_{1}<\ldots<i_{k} \leqslant N\right\}$ de $L^{\prime}$ tal que

$$
\hat{\omega}^{b}\left(e_{a}^{i_{1} \ldots i_{k}}\right)=e^{i_{1}} \wedge \ldots \wedge e^{i_{k}} \otimes \hat{e}_{a},
$$

e assim, para $1 \leqslant i_{1}<\ldots<i_{k} \leqslant N$ e $1 \leqslant j_{1}<\ldots<j_{k} \leqslant N$, temos

$$
\omega^{b}\left(e_{a}^{i_{1} \ldots i_{k}}, e_{j_{1}}, \ldots, e_{j_{k}}\right)=\delta_{a}^{b} \delta_{j_{1}}^{i_{1}} \ldots \delta_{j_{k}}^{i_{k}} .
$$

Então o vetor

$$
u=e_{N^{\prime}+1}-\frac{1}{k !} \omega^{a}\left(e_{N^{\prime}+1}, e_{i_{1}}, \ldots, e_{i_{k}}\right) e_{a}^{i_{1} \ldots i_{k}}
$$

não pertence ao subespaço $E_{0} \oplus L$ e, para $1 \leqslant j_{1}<\ldots<j_{k} \leqslant N$, satisfaz

$$
\begin{aligned}
& \omega^{b}\left(u, e_{j_{1}}, \ldots, e_{j_{k}}\right) \\
& \quad=\omega^{b}\left(e_{N^{\prime}+1}, e_{j_{1}}, \ldots, e_{j_{k}}\right)-\frac{1}{k !} \omega^{a}\left(e_{N^{\prime}+1}, e_{i_{1}}, \ldots, e_{i_{k}}\right) \omega^{b}\left(e_{a}^{i_{1} \ldots i_{k}}, e_{j_{1}}, \ldots, e_{j_{k}}\right) \\
& \quad=0
\end{aligned}
$$

de modo que o subespaço $E_{0}$ gerado por $e_{1}, \ldots, e_{N^{\prime}}$ sendo $k$-isotrópico, o subespaço $E_{1}$ gerado por $e_{1}, \ldots, e_{N^{\prime}}$ e $u$ também é.

O teorema de Darboux algébrico agora segue trivialmente: seja $\left\{\hat{e}_{a} \mid 1 \leqslant a \leqslant \hat{n}\right\}$ uma base qualquer de $\hat{T}$ com base dual $\left\{\hat{e}^{a} \mid 1 \leqslant a \leqslant \hat{n}\right\}$ de $\hat{T}^{*}$ e $\left\{e_{i} \mid 1 \leqslant i \leqslant N\right\}$ uma base qualquer de um subespaço $k$-isotrópico $E$ complementar a $L$ em $V$, com base dual $\left\{e^{i} \mid 1 \leqslant i \leqslant N\right\}$ de $L^{\perp} \cong E^{*}$. Escolhendo um subespaço qualquer $L^{\prime}$ de $L$ que é complementar a ker $\hat{\omega}$ e utilizando a igualdade (1.43), definimos a base $\left\{e_{a}^{i_{1} \ldots i_{k}} \mid 1 \leqslant a \leqslant \hat{n}, 1 \leqslant i_{1}<\ldots<i_{k} \leqslant N\right\}$ de $L^{\prime}$ por

$$
\hat{\omega}^{b}\left(e_{a}^{i_{1} \ldots i_{k}}\right)=e^{i_{1}} \wedge \ldots \wedge e^{i_{k}} \otimes \hat{e}_{a}
$$


É fácil ver que a união destas duas bases forma uma base canônica de $L^{\prime} \oplus E$, que é um subespaço de $V$ complementar a ker $\hat{\omega}$. Desta maneira provamos o seguinte

Teorema 1.9 (Teorema de Darboux para espaços vetoriais polilagrangeanos)

Todo espaço polilagrangeano possui uma base canônica polilagrangeana.

Claramente, a construção indutiva de um subespaço $k$-isotrópico $E$ complementar ao subespaço polilagrangeano $L$ apresentada na demonstração do Teorema 1.8 providencia um método iterativo e explícito para a construção de bases polilagrangeanas que constitui uma generalização natural do processo de ortonormalização de Gram-Schmidt.

\subsection{Espaços vetoriais multilagrangeanos}

Nesta seção, mostraremos que toda forma multilagrangeana admite uma base de Darboux, ou base canònica, na qual é representada por uma fórmula do tipo dada na equação (1.83) da Seção 1.4.

Definição 1.6 Sejam $W$ um espaço vetorial de dimensão finita e $V$ um subespaço fixo de $W$, com $n=\operatorname{dim}(W / V)$, e seja $\omega$ uma $(k+1)$-forma multilagrangeana em $W$ de posto $N$ e grau de horizontalidade $k+1-r$, onde $1 \leqslant r \leqslant k$ e $k+1-r \leqslant n$, com subespaço multilagrangeano $L$ de $V$. Uma base de Darboux multilagrangeana ou base canônica multilagrangeana é uma base

$$
\left\{e_{i}, e_{\mu}, e^{i_{1} \ldots i_{s} ; \mu_{1} \ldots \mu_{k-s}} \mid 0 \leqslant s \leqslant r-1, \begin{array}{c}
1 \leqslant i \leqslant N, 1 \leqslant i_{1}<\ldots<i_{s} \leqslant N \\
1 \leqslant \mu \leqslant n, 1 \leqslant \mu_{1}<\ldots<\mu_{k-s} \leqslant n
\end{array}\right\}
$$

de um subespaço de $W$ complementar a ker $\omega$, com base dual

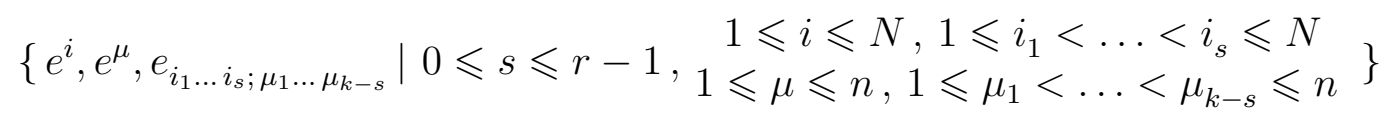

do subespaço $\operatorname{supp} \omega=(\operatorname{ker} \omega)^{\perp}$ de $W^{*}$, tal que

$$
\omega=\sum_{s=0}^{r-1} \frac{1}{s !} \frac{1}{(k-s) !} e_{i_{1} \ldots i_{s} ; \mu_{1} \ldots \mu_{k-s}} \wedge e^{i_{1}} \wedge \ldots \wedge e^{i_{s}} \wedge e^{\mu_{1}} \wedge \ldots \wedge e^{\mu_{k-s}} .
$$

Nesta mesma base, temos para o símbolo

$$
\hat{\omega}=\frac{1}{(r-1) !} \frac{1}{(k+1-r) !}\left(e_{i_{1} \ldots i_{r-1} ; \mu_{1} \ldots \mu_{k+1-r}} \wedge e^{i_{1}} \wedge \ldots \wedge e^{i_{r-1}}\right) \otimes\left(e^{\mu_{1}} \wedge \ldots \wedge e^{\mu_{k+1-r}}\right) .
$$

Se $k=n$ e $r=2$ chamaremos tal base de base de Darboux multissimplética ou base canônica multissimplética. 
A existência de uma base canônica para toda forma multilagrangeana decorre da existência de decomposições diretas $W=L \oplus F$ de $W$ e $V=L \oplus E$ de $V$ do tipo apresentado para $W_{0}$ e $V_{0}$ na Seção 1.4 .

Teorema 1.10 Sejam $W$ um espaço vetorial de dimensão finita e $V$ um subespaço fixo de $W$, com $n=\operatorname{dim}(W / V)$, e seja $\omega$ uma $(k+1)$-forma multilagrangeana em $W$ de posto $N$ e grau de horizontalidade $k+1-r$, onde $1 \leqslant r \leqslant k$ e $k+1-r \leqslant n$, com subespaço multilagrangeano $L$ de $V$. Então existe um subespaço $k$-isotrópico $F$ de $W$ tal que o subespaço $E=F \cap V$ de $V$ é $(r-1)$-isotrópico e

$$
W=F \oplus L, \quad V=E \oplus L
$$

DemonstraçÃo. Primeiro construímos um subespaço $E$ de $V$ de dimensão $N$ que é complementar a $L$ em $V$ e $(r-1)$-isotrópico. Se $r=1$ não há nada a provar, pois neste caso o subespaço vertical inteiro $V$ é isotrópico e portanto temos $L=V, E=\{0\}, N=0$. Se $r>1$ aplicamos o Teorema 1.8 ao símbolo $\hat{\omega}$ de $\omega$ para concluírmos que existe um subespaço $E$ de $V$ de dimensão $N$ que é complementar a $L$ em $V$ e $(r-1)$-isotrópico relativo a $\hat{\omega}$. Tendo em vista que o subespaço vertical $V$ inteiro é $r$-isotrópico relativo a $\omega$, segue que $E$ também é $(r-1)$-isotrópico relativo a $\omega$. - Seja agora $F_{0}$ um subespaço de $W$ de dimensão $N+n^{\prime}$ que é $k$-isotrópico relativo a $\omega$ e tal que $F_{0} \cap V=E$. (Por exemplo, se $n^{\prime}=0$, $F_{0}=E$.) Se $n^{\prime}=n$ o teorema já está demonstrado. Caso contrário, escolha uma base $\left\{e_{1}^{E}, \ldots, e_{N}^{E}, e_{1}, \ldots, e_{n}\right\}$ de um subespaço de $W$ complementar a $L$ tal que os primeiros $N$ vetores formam uma base de $E$ e os primeiros $N+n^{\prime}$ vetores formam uma base de $F_{0}$, e denote a correspondente base dual de $L^{\perp}$ por $\left\{e_{E}^{1}, \ldots, e_{E}^{N}, e^{1}, \ldots, e^{n}\right\}$. Provaremos que existe um vetor $u \in W \backslash\left(F_{0} \oplus L\right)$ tal que o subespaço $F_{1}$ gerado por $u$ e $F_{0}$ é $k$-isotrópico e tal que $F_{1} \cap V=E$, e como $\operatorname{dim} F_{1}=N+n^{\prime}+1$, a afirmação do teorema segue por indução. Para tanto, escolhendo um subespaço $L^{\prime}$ qualquer de $L$ complementar a ker $\omega$, podemos usar o fato de $L$ ser multilagrangeano para concluir que existe uma única base

$$
\left\{e^{i_{1} \ldots i_{s} ; \mu_{1} \ldots \mu_{k-s}} \mid 0 \leqslant s \leqslant r-1, \begin{array}{c}
1 \leqslant i_{1}<\ldots<i_{s} \leqslant N \\
1 \leqslant \mu_{1}<\ldots<\mu_{k-s} \leqslant n
\end{array}\right\}
$$

de $L^{\prime}$ tal que

$$
\omega_{V}^{b}\left(e^{i_{1} \ldots i_{s} ; \mu_{1} \ldots \mu_{k-s}}\right)=e_{E}^{i_{1}} \wedge \ldots \wedge e_{E}^{i_{s}} \wedge e^{\mu_{1}} \wedge \ldots \wedge e^{\mu_{k+1-s}}
$$

e assim, para $0 \leqslant s, t \leqslant r-1,1 \leqslant i_{1}<\ldots<i_{s} \leqslant N, 1 \leqslant j_{1}<\ldots<j_{t} \leqslant N$, $1 \leqslant \mu_{1}<\ldots<\mu_{k-s} \leqslant n, 1 \leqslant \nu_{1}<\ldots<\nu_{k-t} \leqslant n$, temos

$$
\omega\left(e^{i_{1} \ldots i_{s} ; \mu_{1} \ldots \mu_{k-s}}, e_{j_{1}}^{E}, \ldots, e_{j_{t}}^{E}, e_{\nu_{1}}, \ldots, e_{\nu_{k-t}}\right)=\left\{\begin{array}{ccc}
0 & \text { se } & s \neq t \\
\delta_{j_{1}}^{i_{1}} \ldots \delta_{j_{s}}^{i_{s}} \delta_{\nu_{1}}^{\mu_{1}} \ldots \delta_{\nu_{k-s}}^{\mu_{k-s}} & \text { se } & s=t
\end{array}\right\} .
$$


Então o vetor

$$
u=e_{n^{\prime}+1}-\sum_{s=0}^{r-1} \frac{1}{s !} \frac{1}{(k-s) !} \omega\left(e_{n^{\prime}+1}, e_{i_{1}}^{E}, \ldots, e_{i_{s}}^{E}, e_{\mu_{1}}, \ldots, e_{\mu_{k-s}}\right) e^{i_{1} \ldots i_{s} ; \mu_{1} \ldots \mu_{k-s}}
$$

não pertence ao subespaço $F_{0} \oplus L$ e, para $0 \leqslant t \leqslant r-1,1 \leqslant j_{1}<\ldots<j_{t} \leqslant N$ e $1 \leqslant \nu_{1}<\ldots<\nu_{k-t} \leqslant n$, satisfaz

$$
\begin{aligned}
& \omega\left(u, e_{j_{1}}^{E}, \ldots, e_{j_{t}}^{E}, e_{\nu_{1}}, \ldots, e_{\nu_{k-t}}\right) \\
& =\omega\left(e_{n^{\prime}+1}, e_{j_{1}}^{E}, \ldots, e_{j_{t}}^{E}, e_{\nu_{1}}, \ldots, e_{\nu_{k-t}}\right) \\
& \quad-\sum_{s=0}^{r-1} \frac{1}{s !} \frac{1}{(k-s) !} \omega\left(e_{n^{\prime}+1}, e_{i_{1}}^{E}, \ldots, e_{i_{s}}^{E}, e_{\mu_{1}}, \ldots, e_{\mu_{k-s}}\right) \\
& \times \omega\left(e^{i_{1} \ldots i_{s} ; \mu_{1} \ldots \mu_{k-s}}, e_{j_{1}}^{E}, \ldots, e_{j_{t}}^{E}, e_{\nu_{1}}, \ldots, e_{\nu_{k-t}}\right) \\
& =0
\end{aligned}
$$

de modo que o subespaço $F_{0}$ gerado por $e_{1}^{E}, \ldots, e_{N}^{E}, e_{1}, \ldots, e_{n^{\prime}}$ sendo $k$-isotrópico, o subespaço $F_{1}$ gerado por $e_{1}^{E}, \ldots, e_{N}^{E}, e_{1}, \ldots, e_{n^{\prime}}$ e $u$ também é.

O teorema de Darboux algébrico agora segue trivialmente: seja $\left\{e_{i}, e_{\mu} \mid 1 \leqslant i \leqslant N\right.$, $1 \leq \mu \leq n\}$ uma base qualquer de um subespaço $k$-isotrópico $F$ complementar a $L$ em $W$, com base dual $\left\{e^{i}, e^{\mu} \mid 1 \leqslant i \leqslant N, 1 \leq \mu \leq n\right\}$ de $L^{\perp} \cong F^{*}$, tal que $\left\{e_{i} \mid 1 \leqslant i \leqslant N\right\}$ é uma base do subespaço $(r-1)$-isotrópico $E=V \cap F$ complementar a $L$ em $V$. Escolhendo um subespaço qualquer $L^{\prime}$ de $L$ que é complementar a ker $\omega$ e utilizando a igualdade (1.59), definimos a base

$$
\left\{e^{i_{1} \ldots i_{s} ; \mu_{1} \ldots \mu_{k-s}} \mid 0 \leqslant s \leqslant r-1, \begin{array}{c}
1 \leqslant i_{1}<\ldots<i_{s} \leqslant N \\
1 \leqslant \mu_{1}<\ldots<\mu_{k-s} \leqslant n
\end{array}\right\}
$$

de $L^{\prime}$ por

$$
\omega_{V}^{b}\left(e^{i_{1} \ldots i_{s} ; \mu_{1} \ldots \mu_{k-s}}\right)=e^{i_{1}} \wedge \ldots \wedge e^{i_{s}} \wedge e^{\mu_{1}} \wedge \ldots \wedge e^{\mu_{k+1-s}} .
$$

É fácil ver que a união destas duas bases forma uma base canônica de $L^{\prime} \oplus F$, que é um subespaço de $W$ complementar a ker $\omega$. Desta maneira provamos o seguinte

\section{Teorema 1.11 (Teorema de Darboux para espaços vetoriais multilagrangeanos)}

Todo espaço multilagrangeano possui uma base canônica multilagrangeana.

Mais uma vez, a construção indutiva de um subespaço $k$-isotrópico $F$ complementar ao subespaço polilagrangeano $L$ apresentada na demonstração do Teorema 1.10 providencia um método iterativo e explícito para a construção de bases multilagrangeanas que constitui uma generalização natural do processo de ortonormalização de Gram-Schmidt. 
CAPÍTUlo 2

\section{Formas polilagrangeanas e multilagrangeanas: teoria diferencial}

Os conceitos algébricos introduzidos no capítulo anterior fundamentam o desenvolvimento de uma teoria diferencial coerente que será apresentada neste capítulo. Como resultado principal, teremos um teorema de Darboux que garante que formas diferenciais que são pontualmente polilagrangeanas ou multilagrangeanas no sentido algébrico admitem bases locais holônomas, ou seja, coordenadas locais canônicas, se e somente se satisfazem a condição padrão de integrabilidade de serem fechadas.

Quanto à terminologia a ser empregada, faremos uso livre das noções padrão da teoria das variedades e dos fibrados (em particular, dos fibrados vetoriais). Um resultado típico que será bastante usado é o seguinte:

Lema 2.1 Sejam $E_{1}$ e $E_{2}$ subfibrados vetoriais de um fibrado vetorial E. Então $E_{1} \cap E_{2}$ tem dimensão constante se e somente se $E_{1}+E_{2}$ tem dimensão constante e, neste caso, $E_{1} \cap E_{2}$ e $E_{1}+E_{2}$ também são subfibrados vetoriais de $E$.

Uma afirmação análoga vale para intersecções e somas de um número finito arbitrário de subfibrados vetoriais de um fibrado vetorial dado.

Como caso particular, temos o conceito de uma distribuição em uma variedade $P$, que é simplesmente um subfibrado vetorial $D$ do fibrado tangente $T P$ de $P$, de modo a definir uma família de subespaços $D_{p}$ dos espaços tangentes $T_{p} P$ parametrizada pelos pontos $p$ de $P$ tal que os espaços $D_{p}$ "dependem suavemente de $p$ " e "tem dimensão constante" (i.e., $\operatorname{dim} D_{p}$ independe de $p$ ). Tal distribuição é involutiva se para quaisquer dois campos vetoriais $X$ e $Y$ que são seções de $D$, o seu colchete de Lie $[X, Y]$ também é uma seção de $D$, e é integrável se provém de uma folheação de $P$, i.e., uma decomposição de $P$ na união disjunta de subvariedades chamadas de folhas tal que em todo ponto de $P$, o espaço $D_{p}$ é exatamente 
o espaço tangente à folha que passa por $p$. Segundo o teorema de Frobenius, distribuições integráveis são involutivas e, reciprocamente, distribuições involutivas são integráveis.

Um exemplo bem conhecido de uma distribuição involutiva é o núcleo de uma forma diferencial fechada e de posto constante. Brevemente, o núcleo ker $\omega$ de uma $(k+1)$ forma $\omega$ em uma variedade $P$, definido por

$$
\operatorname{ker}_{p} \omega=\operatorname{ker} \omega_{p}=\left\{u_{p} \in T_{p} P \mid \mathrm{i}_{u_{p}} \omega=0\right\}
$$

define uma distribuição em $P$ desde que tenha dimensão constante, i.e., desde que $\omega$ tenha posto constante. ${ }^{1}$ Usando a fórmula geral para a derivada exterior de $\omega$,

$$
\begin{aligned}
d \omega( & \left.X_{1}, \ldots, X_{k+2}\right) \\
= & \sum_{i=1}^{k+2}(-1)^{i-1} X_{i} \cdot\left(\omega\left(X_{1}, \ldots, \hat{X}_{i}, \ldots, X_{k+2}\right)\right) \\
& \quad+\sum_{1 \leqslant i<j \leqslant k+2}(-1)^{i+j} \omega\left(\left[X_{i}, X_{j}\right], X_{1}, \ldots, \hat{X}_{i}, \ldots, \hat{X}_{j}, \ldots, X_{k+2}\right)
\end{aligned}
$$

concluímos que se $d \omega=0$ e $X_{1}$ e $X_{2}$ assumem seus valores em ker $\omega$, então $\left[X_{1}, X_{2}\right]$ também assume seus valores em ker $\omega$, ou seja,

$$
d \omega=0 \Longrightarrow \operatorname{ker} \omega \text { involutivo . }
$$

O mesmo argumento funciona para formas diferenciais a valores vetoriais.

\subsection{Cálculo de Cartan para formas verticais}

Para podermos transferir as estruturas estudadas no primeiro capítulo (mais especificamente, o conceito de símbolo) do âmbito puramente algébrico para o âmbito da geometria diferencial, precisaremos de uma variação do cálculo de Cartan, que na sua forma padrão trata de formas diferenciais em variedades, para podermos lidar com formas diferenciais verticais em espaços totais de fibrados.

Sejam $E$ um fibrado geral sobre uma variedade base $M$, com projeção $\pi: E \rightarrow M$, e $\hat{T}$ um fibrado vetorial sobre a mesma variedade base $M$, com projeção $\hat{\tau}: \hat{T} \rightarrow M$. Considere o "pull-back" de $\hat{T}$ para $E$, que será denotado por $\pi^{*} \hat{T}$, e o fibrado vertical $V E$ de $E$ : ambos são fibrados vetoriais sobre $E$. Então o fibrado vetorial

$$
\bigwedge^{r} V^{*} E \otimes \pi^{*} \hat{T}
$$

\footnotetext{
${ }^{1}$ Apesar de existirem diferentes convenções para o conceito de posto (a mais comum sendo a codimensão do núcleo), a noção de posto constante é unicamente definida.
} 
será chamado o fibrado das $r$-formas verticais sobre $E$, e as suas seções são chamadas $r$ formas diferenciais ou simplesmente $r$-formas sobre $E$, a valores ou com coeficientes em $\pi^{*} \hat{T}$ : o espaço de tais formas será denotado por $\Omega_{V}^{r}\left(E ; \pi^{*} \hat{T}\right)$. Por outro lado, as seções do próprio fibrado vertical $V E$ são chamadas campos vetoriais verticais ou simplesmente campos verticais: o espaço de tais campos será denotado por $\mathfrak{X}_{V}(E)$. Obviamente, $\mathfrak{X}_{V}(E)$ e $\Omega_{V}^{r}\left(E ; \pi^{*} \hat{T}\right)$ são módulos (localmente finitos) sobre a álgebra $\mathfrak{F}(E)$ das funções sobre $E$.

Notamos que há um certo abuso de linguagem nesta terminologia, pois os elementos de $\Omega_{V}^{r}\left(E ; \pi^{*} \hat{T}\right)$ são realmente apenas classes de equivalência de $r$-formas diferenciais sobre $E$, pois $\Omega_{V}^{r}\left(E ; \pi^{*} \hat{T}\right)$ não é um subespaço do espaço $\Omega^{r}\left(E ; \pi^{*} \hat{T}\right)$ de todas as $r$-formas diferenciais sobre $E$ mas é seu espaço quociente

$$
\Omega_{V}^{r}\left(E ; \pi^{*} \hat{T}\right)=\Omega^{r}\left(E ; \pi^{*} \hat{T}\right) / \Omega_{r-1}^{r}\left(E ; \pi^{*} \hat{T}\right)
$$

pelo subespaço $\Omega_{r-1}^{r}\left(E ; \pi^{*} \hat{T}\right)$ de todas as $r$-formas diferenciais 1-horizontais sobre $E$.

O aspecto interessante nesta construção é que é possível desenvolver uma variante do cálculo de Cartan comum para formas diferenciais em uma variedade $M$ em que funções em $M$ são substituídas por funções $f$ em $E$, campos vetoriais em $M$ são substituídos por campos verticais $X$ em $E$ e formas diferenciais em $M$ são substituídas por formas diferenciais verticais $\alpha \mathrm{em} E$ a valores em $\pi^{*} \hat{T}$, e de tal forma que todas as operações deste cálculo são preservadas e continuam satisfazendo às mesmas regras. (Veja [10, Vol. 1, Probl. 8, p. 313] para o caso especial em que $\hat{T}$ é o fibrado trivial $M \times \mathbb{R}$.) São essas operações a contração

$$
\begin{aligned}
& \text { i }: \mathfrak{X}_{V}(E) \times \Omega_{V}^{r}\left(E ; \pi^{*} \hat{T}\right) \longrightarrow \Omega_{V}^{r-1}\left(E ; \pi^{*} \hat{T}\right) \\
& (X, \alpha) \quad \longmapsto \quad \mathrm{i}_{X} \alpha
\end{aligned}
$$

a derivada de Lie vertical

$$
\begin{aligned}
& L: \mathfrak{X}_{V}(E) \times \Omega_{V}^{r}\left(E ; \pi^{*} \hat{T}\right) \longrightarrow \Omega_{V}^{r}\left(E ; \pi^{*} \hat{T}\right), \\
& (X, \alpha) \quad \longmapsto \quad L_{X} \alpha
\end{aligned}
$$

e a derivada exterior vertical

$$
\begin{array}{ccc}
d_{V}: \Omega_{V}^{r}\left(E ; \pi^{*} \hat{T}\right) & \longrightarrow & \Omega_{V}^{r+1}\left(E ; \pi^{*} \hat{T}\right) \\
\alpha & \longmapsto & d_{V} \alpha
\end{array},
$$

além de várias versões do produto exterior, tal como

$$
\begin{array}{ccc}
\wedge: \Omega_{V}^{r}\left(E ; \pi^{*} \hat{T}\right) \times \Omega_{V}^{s}\left(E ; \pi^{*} \hat{T}^{\prime}\right) & \longrightarrow & \Omega_{V}^{r+s}\left(E ; \pi^{*}\left(\hat{T} \otimes \hat{T}^{\prime}\right)\right) \\
\left(\alpha, \alpha^{\prime}\right) & \longmapsto & \alpha \wedge \alpha^{\prime}
\end{array}
$$


A primeira e a última são operações puramente algébricas que são exatamente as mesmas que no caso usual. Para definir a segunda e a terceira, usamos as mesmas fórmulas como no caso usual, a saber

$$
L_{X} \alpha\left(X_{1}, \ldots, X_{r}\right)=X \cdot\left(\alpha\left(X_{1}, \ldots, X_{r}\right)\right)-\sum_{i=1}^{r} \alpha\left(X_{1}, \ldots,\left[X, X_{i}\right], \ldots, X_{r}\right)
$$

$\mathrm{e}$

$$
\begin{aligned}
d_{V} \alpha\left(X_{0}, \ldots, X_{r}\right)= & \sum_{i=0}^{r}(-1)^{i} X_{i} \cdot\left(\alpha\left(X_{0}, \ldots, \hat{X}_{i}, \ldots, X_{r}\right)\right) \\
& +\sum_{0 \leqslant i<j \leqslant r}(-1)^{i+j} \alpha\left(\left[X_{i}, X_{j}\right], X_{0}, \ldots, \hat{X}_{i}, \ldots, \hat{X}_{j}, \ldots, X_{r}\right)
\end{aligned},
$$

onde $X_{0}, X_{1}, \ldots, X_{r} \in \mathfrak{X}_{V}(E)$, o que faz sentido pelo fato de $V E$ ser uma distribuição involutiva em $E$ (i.e., $\mathfrak{X}_{V}(E)$ é subálgebra de $\mathfrak{X}(E)$ relativo ao colchete de Lie), desde que definirmos corretamente a derivada direcional vertical

$$
\begin{aligned}
& \mathfrak{X}_{V}(E) \times \Gamma\left(\pi^{*} \hat{T}\right) \longrightarrow \Gamma\left(\pi^{*} \hat{T}\right) \\
& (X, \varphi) \longmapsto X \cdot \varphi
\end{aligned}
$$

como um operador $\mathbb{R}$-bilinear que é $\mathfrak{F}(E)$-linear no primeiro argumento e satisfaz uma regra de Leibniz no segundo argumento,

$$
X \cdot(f \varphi)=(X \cdot f) \varphi+f(X \cdot \varphi) .
$$

Explicitamente, para $X \in \mathfrak{X}_{V}(E)$ e $\varphi \in \Gamma\left(\pi^{*} \hat{T}\right), X \cdot \varphi \in \Gamma\left(\pi^{*} \hat{T}\right)$ é definido como a derivada direcional comum de funções a valores vetoriais ao longo das fibras, ou seja, para todo ponto $m$ de $M$, temos que $\left.(X \cdot \varphi)\right|_{E_{m}} \in C^{\infty}\left(E_{m}, \hat{T}_{m}\right)$ é dado em termos de $\left.X\right|_{E_{m}} \in \mathfrak{X}\left(E_{m}\right)$ e $\left.\varphi\right|_{E_{m}} \in C^{\infty}\left(E_{m}, \hat{T}_{m}\right)$ por

$$
\left.(X \cdot \varphi)\right|_{E_{m}}=\left.\left.X\right|_{E_{m}} \cdot \varphi\right|_{E_{m}} .
$$

Note que o colchete de Lie sendo natural sob restrição a subvariedades, vale

$$
X \cdot(Y \cdot \varphi)-Y \cdot(X \cdot \varphi)=[X, Y] \cdot \varphi \quad \text { para } X, Y \in \mathfrak{X}_{V}(E), \varphi \in \Gamma\left(\pi^{*} \hat{T}\right),
$$

o que implica que $d_{V}^{2}=0$. Por outro lado, seções $\varphi$ de $\pi^{*} \hat{T}$ obtidas de seções $\hat{t}$ de $\hat{T}$ por composição com $\pi$ são constantes ao longo das fibras e portanto a sua derivada direcional vertical se anula:

$$
X \cdot(\hat{t} \circ \pi)=0 \quad \text { para } X \in \mathfrak{X}_{V}(E), \hat{t} \in \Gamma(\hat{T}) .
$$

Do mesma modo, quando substituírmos $\hat{T}$ por $\hat{T}^{*}$, obtemos

$$
X \cdot\left(\hat{t}^{*} \circ \pi\right)=0 \quad \text { para } X \in \mathfrak{X}_{V}(E), \hat{t}^{*} \in \Gamma\left(\hat{T}^{*}\right),
$$


o que significa que

$$
X \cdot\left\langle\hat{t}^{*} \circ \pi, \varphi\right\rangle=\left\langle\hat{t}^{*} \circ \pi,(X \cdot \varphi)\right\rangle \quad \text { para } X \in \mathfrak{X}_{V}(E), \varphi \in \Gamma\left(\pi^{*} \hat{T}\right) .
$$

Mais geralmente, dada uma $r$-forma $\hat{\omega}$ vertical em $P$ a valores em $\pi^{*} \hat{T}$ e uma seção qualquer $\hat{t}^{*}$ do fibrado dual $\hat{T}^{*}$ de $\hat{T}$, definimos a projeção de $\hat{\omega}$ ao longo de $\hat{t}^{*}$ como sendo a $r$-forma $\hat{\omega}_{\hat{t}^{*}}$ vertical em $P$ comum dada por ${ }^{2}$

$$
\hat{\omega}_{\hat{t}^{*}}(p)=\left\langle\hat{t}^{*}(\pi(p)), \hat{\omega}(p)\right\rangle \quad \text { para } p \in P
$$

e obtemos

$$
d_{V} \hat{\omega}_{\hat{t}^{*}}=\left(d_{V} \hat{\omega}\right)_{\hat{t}^{*}} .
$$

Assim, se $\hat{\omega}$ for fechada, $\hat{\omega}_{\hat{t}^{*}}$ também o será. Ademais, o núcleo ker $\hat{\omega}$ de $\hat{\omega}$ e o núcleo ker $\hat{\omega}_{\hat{t}^{*}}$ de $\hat{\omega}_{\hat{t}^{*}}$, definidos por

$$
\operatorname{ker}_{p} \hat{\omega}=\operatorname{ker} \hat{\omega}_{p}=\left\{u_{p} \in V_{p} P \mid \mathrm{i}_{u_{p}} \hat{\omega}=0\right\} \quad \text { para } p \in P,
$$

e

$$
\operatorname{ker}_{p} \hat{\omega}_{\hat{t}^{*}}=\operatorname{ker}\left(\hat{\omega}_{\hat{t}^{*}}\right)_{p}=\left\{u_{p} \in V_{p} P \mid \mathrm{i}_{u_{p}} \hat{\omega}_{\hat{t}^{*}}=0\right\} \quad \text { para } p \in P,
$$

definem distribuições em $P$ contidas no fibrado vertical $V P$ de $P$ desde que tenham dimensão constante (o que no caso de ker $\hat{\omega}$ equivale à condição de que $\hat{\omega}$ tenha posto constante), com ker $\hat{\omega} \subset \operatorname{ker} \hat{\omega}_{\hat{t}^{*}}$, e aplicando o mesmo argumento como antes, podemos concluir que

$$
d_{V} \hat{\omega}=0 \Longrightarrow \text { ker } \hat{\omega} \text { e ker } \hat{\omega}_{\hat{t}^{*}} \text { involutivo . }
$$

\subsection{Variedades polilagrangeanas}

Nesta seção e na próxima, mostraremos como transferir as estruturas poli- e multilagrangeanas do primeiro capítulo do contexto puramente algébrico, pontual, para o contexto diferencial, adicionando a hipótese adequada de integrabilidade.

Definição 2.1 Uma variedade polilagrangeana é uma variedade $P$ munida de uma $(k+1)$-forma $\hat{\omega}$ a valores em um espaço vetorial fixo $\hat{T}$ de dimensão $\hat{n}$, chamada de forma polilagrangeana de posto $N$, que é fechada,

$$
d \hat{\omega}=0
$$

e que admite uma distribuição L em P, chamada de distribuição polilagrangeana, tal que em todo ponto $p$ de $P$, $\hat{\omega}_{p}$ é uma forma polilagrangeana de posto $N$ em $T_{p} P$ com subespaço polilagrangeano $L_{p}$.

Quando $k=1$, dizemos que $P$ é uma variedade polissimplética e $\hat{\omega}$ é uma forma polissimplética.

\footnotetext{
${ }^{2}$ É importante que $\hat{t}^{*}$ seja uma seção de $\hat{T}^{*}$ e não de $\pi^{*} \hat{T}^{*}$.
} 
Note que conforme o Teorema 1.2 e, em particular, a equação (1.47), $L$ tem dimensão constante se e somente se ker $\hat{\omega}$ tiver dimensão constante, ou seja, $\hat{\omega}$ tiver posto constante.

Como no caso algébrico, introduzimos, para cada forma linear $\hat{t}^{*} \in \hat{T}^{*}$ no espaço auxiliar $\hat{T}$, uma $(k+1)$-forma comum $\omega_{\hat{t}^{*}}$ em $P$ chamada a projeção de $\hat{\omega}$ ao longo de $\hat{t}^{*} \mathrm{e}$ definida por

$$
\omega_{\hat{t}^{*}}=\left\langle\hat{t}^{*}, \hat{\omega}\right\rangle,
$$

e, ao tomarmos uma base $\left\{\hat{e}_{1}, \ldots, \hat{e}_{\hat{n}}\right\}$ de $\hat{T}$, com base dual $\left\{\hat{e}^{1}, \ldots, \hat{e}^{\hat{n}}\right\}$ de $\hat{T}^{*}$, e definirmos as $(k+1)$-formas comuns

$$
\omega^{a}=\omega_{\hat{e}^{a}} \quad(1 \leqslant a \leqslant \hat{n}),
$$

obtemos, como no caso algébrico,

$$
\operatorname{ker} \hat{\omega}=\bigcap_{\hat{t}^{*} \in \hat{T}^{*}} \operatorname{ker} \omega_{\hat{t}^{*}}=\bigcap_{a=1}^{\hat{n}} \operatorname{ker} \omega^{a} .
$$

Quando $\hat{n} \equiv \operatorname{dim} \hat{T} \geqslant 2$, podemos usar o Teorema 1.4 para concluir que

$$
L=\sum_{\hat{t}^{*} \in \hat{T}^{*} \backslash\{0\}} \operatorname{ker} \omega_{\hat{t}^{*}}
$$

Neste caso, é suficiente supor que $\hat{\omega}$ seja uma $(k+1)$-forma a valores em $\hat{T}$ de posto constante e tal que em todo ponto $p$ de $P, L_{p}$ seja o subespaço polilagrangeano de $T_{p} P$ relativo a $\hat{\omega}_{p}$, pois então as fórmulas de dimensão (1.46) e (1.51) garantem que, para todo $1 \leqslant a \leqslant \hat{n}$, ker $\omega^{a}$ têm dimensão constante e portanto define uma distribuição em $P$; logo, $L$ também é uma distribuição em $P$. Ademais, para todo $1 \leqslant a \leqslant \hat{n}, K_{a}$ tem dimensão constante e pode ser escolhido de modo a definir uma distribuição em $P$. Contudo, vale ressaltar que este argumento falha se $\hat{n} \equiv \operatorname{dim} \hat{T}=1$, ou seja, para formas comuns - notavelmente no caso simplético, onde a distribuição lagrangeana está longe de ser única e uma mera coleção de subespaços lagrangeanos não precisa ser suave.

Quando $\hat{n} \equiv \operatorname{dim} \hat{T} \geqslant 3$, podemos ainda aplicar o mesmo Teorema 1.4 para mostrar que a distribuição polilagrangeana é necessariamente involutiva!

Teorema 2.1 Seja $P$ uma variedade polilagrangeana com $(k+1)$-forma polilagrangeana $\hat{\omega}$ a valores em um espaço vetorial $\hat{T}$ de dimensão $\hat{n} \equiv \operatorname{dim} \hat{T} \geqslant 3$. Então a distribuição polilagrangeana $L$ é involutiva.

Demonstração. Continuando a usar a notação anterior, suponha que $X$ e $Y$ sejam campos vetoriais que são seções de $L$. Usando a decomposição

$$
L=K_{0} \oplus K_{1} \oplus \ldots \oplus K_{\hat{n}},
$$


com $K_{0}=\operatorname{ker} \hat{\omega}$ (veja a equação (1.48)), podemos decompor $X$ e $Y$ conforme

$$
X=\sum_{a=0}^{\hat{n}} X_{a} \quad, \quad Y=\sum_{b=0}^{\hat{n}} Y_{b},
$$

onde $X_{a}$ e $Y_{b}$ são, respectivamente, seções de $K_{a}$ e $K_{b}$. Como $\hat{n} \geqslant 3$, sempre podemos para cada valor de $a$ e $b$ encontrar um terceiro valor $c$ tal que $c \neq a, c \neq b$ e $c \neq 0$; então $K_{a} \subset \operatorname{ker} \omega^{c}, K_{b} \subset \operatorname{ker} \omega^{c}$ e, como $\omega^{c}$ é fechada de posto constante e portanto ker $\omega^{c}$ é involutivo, concluir que o campo vetorial $\left[X_{a}, Y_{b}\right]$ é uma seção de $\operatorname{ker} \omega^{c} \subset L$.

Existem variedades polilagrangeanas cuja distribuição polilagrangeana não é involutiva. Segundo o Teorema 2.1, isto só pode ocorrer quando $\hat{n}=1$ ou $\hat{n}=2$. Um exemplo interessante de estrutura polilagrangeana não integrável é dado pela forma polissimplética do seguinte exemplo:

Exemplo 2.1 Seja $P=S^{3}=S U(2), \hat{T}=\mathbb{R}^{2}$ e defina $\alpha^{1}, \alpha^{2}, \alpha^{3}$ como sendo as três componentes da forma invariante à esquerda de Maurer-Cartan em $P$ com respeito à base canônica de $\mathbb{R}^{3}$, que são duais aos três campos invariantes à esquerda $\xi_{1}, \xi_{2}$ e $\xi_{3}$ gerados por esta base canônica. Defina

$$
\omega^{(1)}=\alpha^{3} \wedge \alpha^{1} \quad, \quad \omega^{(2)}=\alpha^{3} \wedge \alpha^{2}, \quad \omega^{(3)}=\alpha^{1} \wedge \alpha^{2} .
$$

Segue imediatamente das equações estruturais de Maurer-Cartan que cada uma destas 2formas é fechada. Omitindo $\omega^{(3)}$, por exemplo, vemos que

$$
\hat{\omega}=\omega^{(1)} \hat{e}_{1}+\omega^{(2)} \hat{e}_{2}
$$

é uma forma polissimplética não degenerada de posto 1 em $P$ cuja distribuição polilagrangeana é gerada por $\xi_{1}$ (que gera o núcleo de $\omega^{(2)}$ ) e $\xi_{2}$ (que gera o núcleo de $\omega^{(1)}$ ), e esta definitivamente não é involutiva.

Isso implica que para $\hat{n}=1$ ou $\hat{n}=2$ a condição de integrabilidade da distribuição polilagrangeana $L$ precisa ser imposta separadamente, quando necessário ou conveniente.

\subsection{Fibrados poli- e multilagrangeanos}

Passando agora de variedades para fibrados, suponha que $P$ é um fibrado sobre uma variedade base $M$ com projeção $\pi: P \rightarrow M$ e considere a aplicação tangente $T \pi: T P \rightarrow T M$ cujo 
núcleo é um subfibrado vetorial $V P$ do fibrado tangente $T P$ de $P$ chamado de fibrado vertical, o que nos leva à seguinte sequência exata de fibrados vetoriais sobre $P$ :

$$
0 \longrightarrow V P \longrightarrow T P \stackrel{T \pi}{\longrightarrow} \pi^{*} T M \longrightarrow 0
$$

Esta é o análogo da sequência exata (1.3) de espaços vetoriais do primeiro capítulo, e é a ela que se referem as noções de horizontalidade que aparecerão neste capítulo.

Primeiro, contudo, introduzimos a noção de um fibrado polilagrangeano que formaliza a idéia de uma "família de variedades polilagrangeanas suavemente parametrizada pelos pontos de uma variedade base $M$ ". Para isso, precisaremos dos conceitos introduzidos na primeira seção deste capítulo.

Definição 2.2 Um fibrado polilagrangeano é um fibrado $P$ sobre uma variedade $M$ de dimensão $n$ munido de uma r-forma vertical $\hat{\omega}$ no espaço total $P$ a valores em um fibrado vetorial fixo $\hat{T}$ de dimensão $\hat{n}$ sobre a mesma variedade $M$, chamada de forma polilagrangeana ao longo das fibras de $P$, ou simplesmente forma polilagrangeana, de posto $N$, que é verticalmente fechada,

$$
d_{V} \hat{\omega}=0
$$

e que admite uma distribuição $L$ em $P$ contida no fibrado vertical $V P$ de $P$, chamada de distribuição polilagrangeana, tal que em todo ponto $p$ de $P$, $\omega_{p}$ é uma forma polilagrangeana de posto $N$ em $V_{p} P$ com subespaço polilagrangeano $L_{p}$.

Quando $r=2$, dizemos que $P$ é um fibrado polissimplético e $\hat{\omega}$ é uma forma polissimplética ao longo das fibras de P.

Obviamente, se $P$ é um fibrado polilagrangeano sobre uma variedade $M$ com forma polilagrangeana $\hat{\omega}$, a valores em $\hat{T}$, então para todo ponto $m$ de $M$, a fibra $P_{m}$ de $P$ sobre $m$ será uma variedade polilagrangeana cuja forma polilagrangeana $\hat{\omega}_{m}$, com valores em $\hat{T}_{m}$, é a restrição de $\hat{\omega}$ a $P_{m}$ e cuja distribuição polilagrangeana $L_{m}$ é a restrição de $L$ a $P_{m}$. A definição dada acima serve principalmente para garantir a regularidade da família de variedades polilagrangeanas assim definida, parametrizada pelos pontos $m$ de $M$, ao longo de $M$, ou seja, nas direções transversais às fibras. Obviamente o teorema de involutividade (Teorema 2.1) continua valendo neste contexto.

De forma análoga, para estruturas multilagrangeanas adotamos a seguinte

Definição 2.3 Um fibrado multilagrangeano é um fibrado $P$ sobre uma variedade $M$ de dimensão $n$ munido de uma $(k+1)$-forma $\omega$ no espaço total $P$, chamada de forma multilagrangeana de posto $N$ e grau de horizontalidade $k+1-r$, onde $1 \leqslant r \leqslant k$ e $k+1-r \leqslant n$, que é fechada,

$$
d \omega=0
$$


e que admite uma distribuição $L$ em $P$, chamada de distribuição multilagrangeana, tal que em todo ponto $p$ de $P, \omega_{p}$ é uma forma multilagrangeana de posto $N$ e grau de horizontalidade $k+1-r$ em $T_{p} P$ com subespaço multilagrangeano $L_{p}$. Quando $k=n$ e $r=2$ e $\omega$ for não-degenerada, dizemos que $P$ é um fibrado multissimplético e $\omega$ é uma forma multissimplética.

\subsection{O símbolo}

A seguir, queremos mostrar como a estreita relação entre estruturas multilagrangeanas e estruturas polilagrangeanas exibida no primeiro capítulo pode ser transferida do âmbito puramente algébrico para o âmbito da geometria diferencial: aqui também existe um conceito de símbolo que a cada forma multilagrangeana em um fibrado associa uma forma polilagrangeana neste fibrado, a valores em um fibrado de formas na variedade base. Para isso, precisaremos mais uma vez dos conceitos introduzidos na primeira seção deste capítulo.

Para tanto, suporemos como na seção anterior que $P$ é um fibrado sobre uma variedade base $M$ de dimensão $n$ e faremos uso da sequência exata (2.24). Também suporemos como na seção anterior que $\omega$ é uma $(k+1)$-forma em $P$ que é $(k+1-r)$-horizontal, onde $1 \leqslant r \leqslant k$, e $r \geqslant k+1-n$ caso $k>n$ :

$$
\omega \in \Omega_{r}^{k+1}(P)=\Gamma\left(\bigwedge_{r}^{k+1} T^{*} P\right) .
$$

Explicitamente, isso significa que a contração de $\omega$ com mais de $r$ campos verticais em $P$ se anula:

$$
\mathrm{i}_{X_{1}} \ldots \mathrm{i}_{X_{r+1}} \omega=0 \quad \text { para } X_{1}, \ldots, X_{r+1} \in \mathfrak{X}_{V}(P) .
$$

Então o símbolo $\hat{\omega}$ de $\omega$, definido pontualmente (i.e., tal que em todo ponto $p$ de $P$, $\hat{\omega}_{p}$ é o símbolo de $\omega_{p}$ no sentido do primeiro capítulo) será uma $r$-forma vertical em $P$ a valores no fibrado vetorial $\pi^{*}\left(\bigwedge^{k+1-r} T^{*} M\right)$ :

$$
\hat{\omega} \in \Omega_{V}^{r}\left(P, \pi^{*}\left(\bigwedge^{k+1-r} T^{*} M\right)\right)=\Gamma\left(\bigwedge^{r} V^{*} P \otimes \pi^{*}\left(\bigwedge^{k+1-r} T^{*} M\right)\right) .
$$

Usando o isomorfismo canônico $\pi^{*}\left(\bigwedge^{k+1-r} T^{*} M\right) \cong \bigwedge_{0}^{k+1-r} T^{*} P$ de fibrados vetoriais sobre $P$ como identificação, temos

$$
\hat{\omega}\left(X_{1}, \ldots, X_{r}\right)=\mathrm{i}_{X_{1}} \ldots \mathrm{i}_{X_{r}} \omega \quad \text { para } X_{1}, \ldots, X_{r} \in \mathfrak{X}_{V}(P) .
$$

Mais explicitamente, usando o levantamento horizontal

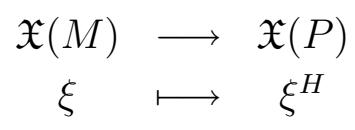


de campos vetoriais induzido por uma conexão qualquer em $P$, temos

$$
\begin{gathered}
\hat{\omega}\left(X_{1}, \ldots, X_{r}\right)\left(\xi_{1} \circ \pi, \ldots, \xi_{k+1-r} \circ \pi\right)=\omega\left(X_{1}, \ldots, X_{r}, \xi_{1}^{H}, \ldots, \xi_{k+1-r}^{H}\right) \\
\operatorname{para} X_{1}, \ldots, X_{r} \in \mathfrak{X}_{V}(P), \xi_{1}, \ldots, \xi_{k+1-r} \in \mathfrak{X}(M),
\end{gathered}
$$

notando que apesar de que a composição de campos vetoriais em $M$ com a projeção $\pi$ fornece apenas um subespaço do espaço de todas as seções do pull-back $\pi^{*} T M$ de $T M$ por $\pi$, esta fórmula é suficiente para determinar completamente o valor de $\hat{\omega}$ em cada ponto de $P$.

Teorema 2.2 Com as notações acima, suponha que a forma $\omega$ seja tal que

$$
d \omega \in \Omega_{r}^{k+2}(P)=\Gamma\left(\bigwedge_{r}^{k+2} T^{*} P\right),
$$

i.e., dw é $(k+2-r)$-horizontal. Então $\hat{\omega}$ é verticalmente fechada:

$$
d_{V} \hat{\omega}=0 .
$$

Em particular, para que $\hat{\omega}$ seja fechada, é suficiente mas não é necessário que $\omega$ seja fechada.

DemonstraÇÃo. Sejam $X_{0}, \ldots, X_{r}$ campos verticais em $P$ e $\xi_{1}, \ldots, \xi_{k+1-r}$ campos vetoriais em $M$ com os seus respectivos levantamentos horizontais $\xi_{1}^{H}, \ldots, \xi_{k+1-r}^{H}$ em relação a uma conexão qualquer em $P$. Então temos

$$
\begin{aligned}
\left(d_{V} \hat{\omega}\left(X_{0}, \ldots, X_{r}\right)\right)\left(\xi_{1} \circ \pi, \ldots, \xi_{k+1-r} \circ \pi\right) & \\
= & \left(\sum_{i=0}^{r}(-1)^{i} X_{i} \cdot\left(\hat{\omega}\left(X_{0}, \ldots, \hat{X}_{i}, \ldots, X_{r}\right)\right)\right. \\
& \left.\quad+\sum_{0 \leqslant i<j \leqslant r}(-1)^{i+j} \hat{\omega}\left(\left[X_{i}, X_{j}\right], X_{0}, \ldots, \hat{X}_{i}, \ldots, \hat{X}_{j}, \ldots, X_{r}\right)\right)\left(\xi_{1} \circ \pi, \ldots, \xi_{k+1-r} \circ \pi\right) \\
= & \sum_{i=0}^{r}(-1)^{i} X_{i} \cdot\left(\hat{\omega}\left(X_{0}, \ldots, \hat{X}_{i}, \ldots, X_{r}\right)\left(\xi_{1} \circ \pi, \ldots, \xi_{k+1-r} \circ \pi\right)\right) \\
& \quad+\sum_{0 \leqslant i<j \leqslant r}(-1)^{i+j} \hat{\omega}\left(\left[X_{i}, X_{j}\right], X_{0}, \ldots, \hat{X}_{i}, \ldots, \hat{X}_{j}, \ldots, X_{r}\right)\left(\xi_{1} \circ \pi, \ldots, \xi_{k+1-r} \circ \pi\right) \\
=\sum_{i=0}^{r}(-1)^{i} X_{i} \cdot \omega\left(X_{0}, \ldots, \hat{X}_{i}, \ldots, X_{r}, \xi_{1}^{H}, \ldots, \xi_{k+1-r}^{H}\right) & \quad+\sum_{j=1}^{k+1-r}(-1)^{r+j} \xi_{j}^{H} \cdot \omega\left(X_{0}, \ldots, X_{r}, \xi_{1}^{H}, \ldots, \hat{\xi}_{j}^{H}, \ldots, \xi_{k+1-r}^{H}\right)
\end{aligned}
$$




$$
\begin{aligned}
& +\sum_{0 \leqslant i<j \leqslant r}(-1)^{i+j} \omega\left(\left[X_{i}, X_{j}\right], X_{0}, \ldots, \hat{X}_{i}, \ldots, \hat{X}_{j}, \ldots, X_{r}, \xi_{1}^{H}, \ldots, \xi_{k+1-r}^{H}\right) \\
& +\sum_{i=0}^{r} \sum_{j=1}^{k+1-r}(-1)^{i} \omega\left(\left[X_{i}, \xi_{j}^{H}\right], X_{0}, \ldots, \hat{X}_{i}, \ldots, X_{r}, \xi_{1}^{H}, \ldots, \hat{\xi}_{j}^{H}, \ldots, \xi_{k+1-r}^{H}\right) \\
& +\sum_{1 \leqslant i<j \leqslant k+1-r}(-1)^{i+j} \omega\left(\left[\xi_{i}^{H}, \xi_{j}^{H}\right], X_{0}, \ldots, X_{r}, \xi_{1}^{H}, \ldots, \hat{\xi}_{i}^{H}, \ldots, \hat{\xi}_{j}^{H}, \ldots, \xi_{k+1-r}^{H}\right) \\
& =d \omega\left(X_{0}, \ldots, X_{r}, \xi_{1}^{H}, \ldots, \xi_{k+1-r}^{H}\right)
\end{aligned}
$$

onde, na segunda igualdade, usamos a equação $(2.13) \operatorname{com} \varphi=\hat{\omega}\left(X_{0}, \ldots, \hat{X}_{i}, \ldots, X_{r}\right)$ e $\hat{t}^{*}=\xi_{1} \wedge \ldots \wedge \xi_{k+1-r}$ e na terceira igualdade adicionamos termos que se anulam devido à condição de que $\omega$ é $(k+1-r)$-horizontal, uma vez que os colchetes de Lie $\left[X_{i}, \xi_{j}^{H}\right]$ são verticais. (Mais geralmente, se $X$ for um campo projetável em $P$ e $\pi_{*} X$ for sua projeção, então $X$ e $\pi_{*} X$ são $\pi$-relacionados e $\xi^{H}$ e $\xi$ são $\pi$-relacionados; assim, $\left[X, \xi^{H}\right]$ e $\left[\pi_{*} X, \xi\right]$ também são $\pi$-relacionados. Mas $X$ sendo vertical significa que $\pi_{*} X=0$.)

Como corolário dos Teoremas 1.7 e 2.2, obtemos

Teorema 2.3 Seja $P$ um fibrado sobre uma variedade $M$ de dimensão $n$, com projeção $\pi: P \rightarrow M$, seja $\omega$ uma $(k+1)$-forma em $P$ que é $(k+1-r)$-horizontal, onde $1 \leqslant r \leqslant k$ e $k+1-r \leqslant n$, e seja $\hat{\omega}$ o símbolo de $\omega$, que é uma $r$-forma vertical em $P$ a valores no fibrado das $(k+1-r)$-formas sobre $M$ (ou mais exatamente, em $\pi^{*}\left(\bigwedge^{k+1-r} T^{*} M\right)$ ). Suponha que $\omega$ é multilagrangeana, com distribuição multilagrangeana L. Então $\hat{\omega}$ é polilagrangeana, com distribuição polilagrangeana L. Se $\omega$ for multissimplética então $\hat{\omega}$ será polissimplética.

Quanto à questão da involutividade da distribuição poli- ou multilagrangeana em fibrados, podemos garantir que

$$
L \text { é involutiva se } \hat{n} \geqslant 3 \text {, no caso polilagrangeano , }
$$

$\mathrm{e}$

$$
L \text { é involutiva se }\left(\begin{array}{c}
n \\
k+1-r
\end{array}\right) \geqslant 3 \text {, no caso multilagrangeano , }
$$

sendo que o argumento é exatamente o mesmo que na demonstração do Teorema 2.1, representando $L$ em termos de distribuições involutivas construídas a partir do núcleo da forma polilagrangeana $\hat{\omega}$ e dos núcleos das formas projetadas $\hat{\omega}_{\hat{t}^{*}}$. Para fibrados multilagrangeanos, é interessante especificar melhor quando a distribuição $L$ pode deixar de ser involutiva: 
1. $n=k+1-r$ : isso inclui o caso simplético $(r=2)$ com

$$
\omega \in \Omega_{2}^{n+2}(P) \quad, \quad \hat{\omega} \in \Omega_{V}^{2}\left(P, \pi^{*}\left(\bigwedge^{n} T^{*} M\right)\right)
$$

representando, na hipótese de que ker $\hat{\omega}=\{0\}$, uma "família de formas simpléticas suavemente parametrizada pelos pontos de uma variedade base $M$ ", de dimensão $n$ qualquer. Neste contexto, não é difícil construir exemplos de distribuições lagrangeanas que não são involutivas.

2. $n>k+1-r$ : a única possibilidade compatível com a condição de que $\left(\begin{array}{c}n \\ k+1-r\end{array}\right)=2$ é $n=2$ e $k=r$ : isso inclui o caso multissimplético com variedade base $M$ bidimensional $(n=k=r=2)$. Um exemplo explícito desta situação pode ser obtido a partir da seguinte construção, que constitui uma adaptação do Exemplo 2.1.

Exemplo 2.2 Seja $M$ uma variedade bidimensional que admite 1-formas fechadas $\theta_{1}$ e $\theta_{2}$ tais que $\theta_{1} \wedge \theta_{2}$ é uma forma de volume para $M$ (por exemplo, o toro bidimensional ou um dos seus recobrimentos), e seja $P$ o espaço total de um fibrado principal sobre $M$ com grupo estrutural $U(2)$. Dada uma forma de conexão $A \in \Omega^{1}(P, \mathfrak{u}(2))$ sobre $P$ com forma de curvatura $F \in \Omega^{2}(P, \mathfrak{u}(2))$ e usando a base $\left\{i 1, \sigma_{1} / 2 i, \sigma_{2} / 2 i, \sigma_{3} / 2 i\right\}$ de $\mathfrak{u}(2)$ onde

$$
\sigma_{1}=\left(\begin{array}{ll}
0 & 1 \\
1 & 0
\end{array}\right), \quad \sigma_{2}=\left(\begin{array}{cc}
0 & -i \\
i & 0
\end{array}\right), \quad \sigma_{3}=\left(\begin{array}{cc}
1 & 0 \\
0 & -1
\end{array}\right)
$$

são as matrizes de Pauli, de modo que

$$
A=A^{0} i 1+A^{a} \frac{\sigma_{a}}{2 i} \quad, \quad F=F^{0} i 1+F^{a} \frac{\sigma_{a}}{2 i}
$$

e

$$
F^{0}=d A^{0} \quad, \quad F^{a}=d A^{a}+\frac{1}{2} \epsilon^{a b c} A^{b} \wedge A^{c}
$$

temos que

$$
\omega=A^{3} \wedge A^{1} \wedge \pi^{*} \theta_{1}+A^{3} \wedge A^{2} \wedge \pi^{*} \theta_{2}-A^{0} \wedge \pi^{*} \theta_{1} \wedge \pi^{*} \theta_{2}
$$

é uma forma multissimplética não degenerada de posto 1 em $P$ cuja distribuição multilagrangeana é gerada pelos campos fundamentais em $P$ associados aos geradores $\sigma_{1} / 2 i$, $\sigma_{2} / 2 i$ e $i 1$, que não é involutiva. De fato, se definirmos $L$ como sendo o subfibrado vetorial de $V P$ gerado por estes campos fundamentais e observarmos que $V P$ é o subfibrado vetorial de $T P$ gerado pelos campos fundamentais em $P$ associados aos geradores $\sigma_{1} / 2 i, \sigma_{2} / 2 i, \sigma_{3} / 2 i$ e $i 1$, é imediato que $\omega$ se anula sob contração com três campos vetoriais em $V P$ ou com dois campos vetoriais em $L P$, de modo que resta apenas verificar que $\omega$ é fechada. Isto segue por 
um cálculo direto,

$$
\begin{aligned}
d \omega= & d A^{3} \wedge A^{1} \wedge \pi^{*} \theta_{1}-A^{3} \wedge d A^{1} \wedge \pi^{*} \theta_{1}+A^{3} \wedge A^{1} \wedge \pi^{*}\left(d \theta_{1}\right) \\
& +d A^{3} \wedge A^{2} \wedge \pi^{*} \theta_{2}-A^{3} \wedge d A^{2} \wedge \pi^{*} \theta_{2}+A^{3} \wedge A^{2} \wedge \pi^{*}\left(d \theta_{2}\right) \\
& -d A^{0} \wedge \pi^{*} \theta_{1} \wedge \pi^{*} \theta_{2}+A^{0} \wedge \pi^{*}\left(d \theta_{1}\right) \wedge \pi^{*} \theta_{2}-A^{0} \wedge \pi^{*} \theta_{1} \wedge \pi^{*}\left(d \theta_{2}\right) \\
= & F^{3} \wedge A^{1} \wedge \pi^{*} \theta_{1}-A^{3} \wedge F^{1} \wedge \pi^{*} \theta_{1}+F^{3} \wedge A^{2} \wedge \pi^{*} \theta_{2}-A^{3} \wedge F^{2} \wedge \pi^{*} \theta_{2} \\
& \quad-F^{0} \wedge \pi^{*} \theta_{1} \wedge \pi^{*} \theta_{2},
\end{aligned}
$$

tendo em vista que todos estes termos são (pelo menos) 3-horizontais e portanto se anulam, pois $M$ é apenas bidimensional. Note que o símbolo desta forma multisimplética é a forma polisimplética dada por

$$
\hat{\omega}=\left(\left.\left.A^{3}\right|_{V P} \wedge A^{1}\right|_{V P}\right) \otimes \theta_{1}+\left(\left.\left.A^{3}\right|_{V P} \wedge A^{2}\right|_{V P}\right) \otimes \theta_{2}
$$

que é essencialmente a forma polissimplética do Exemplo 2.1 (trivialmente estendida de $S U(2)$ para $U(2))$.

\subsection{O teorema de Darboux}

Agora estamos aptos a provar o teorema de Darboux para formas poli- e multilagrangeanas. A estrutura algébrica específica dos subespaços poli- e multilagrangeanos identificada no primeiro capítulo mostra-se crucial, fazendo com que este teorema central pode, em todos os casos, ser demonstrado por uma adaptação adequada do procedimento usado para provar o teorema de Darboux clássico em variedades simpléticas. (Veja, por exemplo, [1]).

Teorema 2.4 (Teorema de Darboux para variedades polilagrangeanas) Seja $P$ uma variedade polilagrangeana com $(k+1)$-forma polilagrangeana $\hat{\omega}$ de posto $N$ a valores em um espaço vetorial fixo $\hat{T}$ de dimensão $\hat{n}$ e com distribuição polilagrangeana $L$ involutiva (o que é automático se $\hat{n} \geqslant 3$ ), e seja $\left\{\hat{e}_{a} \mid 1 \leqslant a \leqslant \hat{n}\right\}$ uma base qualquer de $\hat{T}$. Então existe em torno de cada ponto de $P$ um sistema de coordenadas locais $\left(q^{i}, p_{i_{1} \ldots i_{k}}^{a}, r^{\kappa}\right)(1 \leqslant a \leqslant \hat{n}$, $1 \leqslant i \leqslant N, 1 \leqslant i_{1}<\ldots<i_{k} \leqslant N, 1 \leqslant \kappa \leqslant \operatorname{dim}$ ker $\left.\hat{\omega}\right)$, chamadas de coordenadas de Darboux ou coordenadas canônicas, tais que

$$
\hat{\omega}=\frac{1}{k !} d p_{i_{1} \ldots i_{k}}^{a} \wedge d q^{i_{1}} \wedge \ldots \wedge d q^{i_{k}} \otimes \hat{e}_{a}
$$

sendo que $L$ é gerado pelos campos vetoriais $\partial / \partial p_{i_{1} \ldots i_{k}}^{a}$ e $\partial / \partial r^{\kappa}$ enquanto que ker $\hat{\omega}$ é gerado pelos campos vetoriais $\partial / \partial r^{\kappa}$. 
Teorema 2.5 (Teorema de Darboux para fibrados polilagrangeanos) Seja $\quad P$ um fibrado polilagrangeano sobre uma variedade $M$ de dimensão $n$ com $r$-forma vertical polilagrangeana $\hat{\omega}$ de posto $N$ a valores em um fibrado vetorial fixo $\hat{T}$ de dimensão $\hat{n}$ sobre a mesma variedade $M$ e com distribuição polilagrangeana $L$ involutiva (o que é automático se $\hat{n} \geqslant 3)$, e seja $\left\{\hat{e}_{a} \mid 1 \leqslant a \leqslant \hat{n}\right\}$ uma base qualquer de seções locais de $\hat{T}$. Então existe em torno de cada ponto de $P$ (no dominio de definição da referida base de seções locais) um sistema de coordenadas locais $\left(x^{\mu}, q^{i}, p_{i_{1} \ldots i_{r-1}}^{a}, r^{\kappa}\right)(1 \leqslant \mu \leqslant n, 1 \leqslant a \leqslant \hat{n}, 1 \leqslant i \leqslant N$, $1 \leqslant i_{1}<\ldots<i_{r-1} \leqslant N, 1 \leqslant \kappa \leqslant \operatorname{dim}$ ker $\left.\hat{\omega}\right)$, chamadas de coordenadas de Darboux ou coordenadas canônicas, tais que

$$
\hat{\omega}=\frac{1}{(r-1) !} d p_{i_{1} \ldots i_{r-1}}^{a} \wedge d q^{i_{1}} \wedge \ldots \wedge d q^{i_{r-1}} \otimes \hat{e}_{a},
$$

sendo que $L$ é gerado pelos campos vetoriais $\partial / \partial p_{i_{1} \ldots i_{r-1}}^{a}$ e $\partial / \partial r^{\kappa}$ enquanto que ker $\hat{\omega}$ é gerado pelos campos vetoriais $\partial / \partial r^{\kappa}$.

Teorema 2.6 (Teorema de Darboux multilagrangeano) Seja $P$ um fibrado multilagrangeano sobre uma variedade $M$ de dimensão $n$ com $(k+1)$-forma multilagrangeana $\omega$ de posto $N$ e grau de horizontalidade $k+1-r$, onde $1 \leqslant r \leqslant k$ e $k+1-r \leqslant n$, e com distribuição multilagrangeana L involutiva (o que é automático se $\left(\begin{array}{c}n \\ k+1-r\end{array}\right) \geqslant 3$ ). Então existe em torno de cada ponto de $P$ um sistema de coordenadas locais $\left(x^{\mu}, q^{i}, p_{i_{1} \ldots i_{s} ; \mu_{1} \ldots \mu_{k-s}}, r^{\kappa}\right)(0 \leqslant s \leqslant r-1$, $1 \leqslant \mu \leqslant n, 1 \leqslant i \leqslant N, 1 \leqslant i_{1}<\ldots<i_{s} \leqslant N, 1 \leqslant \mu_{1}<\ldots<\mu_{k-s} \leqslant N$, $1 \leqslant \kappa \leqslant \operatorname{dim}$ ker $\omega$ ), chamadas de coordenadas de Darboux ou coordenadas canônicas, tais que

$$
\omega=\sum_{s=0}^{r-1} \frac{1}{s !} \frac{1}{(k-s) !} d p_{i_{1} \ldots i_{s} ; \mu_{1} \ldots \mu_{k-s}} \wedge d q^{i_{1}} \wedge \ldots \wedge d q^{i_{s}} \wedge d x^{\mu_{1}} \wedge \ldots \wedge d x^{\mu_{k-s}},
$$

sendo que $L$ é gerado pelos campos vetoriais $\partial / \partial p_{i_{1} \ldots i_{s} ; \mu_{1} \ldots \mu_{k-s}} e \partial / \partial r^{\kappa}$ enquanto que ker $\omega$ é gerado pelos campos vetoriais $\partial / \partial r^{\kappa}$. Nestas mesmas coordenadas, temos para o símbolo

$$
\hat{\omega}=\frac{1}{(r-1) !} \frac{1}{(k+1-r) !}\left(d p_{i_{1} \ldots i_{r-1} ; \mu_{1} \ldots \mu_{k+1-r}} \wedge d q^{i_{1}} \wedge \ldots \wedge d q^{i_{r-1}}\right) \otimes\left(d x^{\mu_{1}} \wedge \ldots \wedge d x^{\mu_{k+1-r}}\right) .
$$

DemonstraÇÃo. Para simplificar a apresentação, concentrar-nos-emos no caso multilagrangeano: a prova nos outros dois casos é completamente análoga e requer apenas modificações de menor porte que são mais ou menos óbvias.

Inicialmente, notamos que se trata de um teorema de caráter local, e como o núcleo de $\omega$, o subfibrado multilagrangeano $L$ e o fibrado vertical $V P$ de $P$ são todos involutivos e satisfazem as inclusões ker $\omega \subset L \subset V P$, podemos sem perda de generalidade trabalhar em uma carta local da variedade $P$ em torno do ponto de referência escolhido tal que as folheações 
correspondentes são dadas por subespaços fixos do espaço modelo, i.e., através de um difeomorfismo, temos $P \cong \mathbb{R}^{n} \oplus \mathbb{R}^{N} \oplus L_{0} \oplus K_{0}$ com $V P \cong \mathbb{R}^{N} \oplus L_{0} \oplus K_{0}, L \cong L_{0} \oplus K_{0}$ e ker $\omega \cong K_{0}$. Suporemos ainda que, nesta carta, o acima mencionado ponto de referência corresponde à origem 0 deste espaço vetorial e estenderemos o valor $\omega(0)$ da forma multilagrangeana $\omega$ neste ponto a uma forma multilagrangeana $\omega_{0}$ definida e constante no domínio da carta escolhida; então a existência de uma base de Darboux para $\omega_{0}$, da forma dada pela equação 2.36, já é garantida pelo teorema de Darboux algébrico do primeiro capítulo (Teorema 1.11).

Posto isso, defina, para $t \in \mathbb{R}$, a família de $(k+1)$-formas $\omega_{t}=\omega_{0}+t\left(\omega-\omega_{0}\right)$. Obviamente, temos $\omega_{t}(0)=\omega_{0}$ para todo $t \in \mathbb{R}$, e por definição, esta forma é não-degenerada em $K_{0}^{\prime}=\mathbb{R}^{n} \oplus \mathbb{R}^{N} \oplus L_{0}$ (o complemento de $K_{0} \mathrm{em} P$ ). Como não-degenerscência é uma condição aberta, e usando um argumento de compacidade em relação ao parâmetro $t$, podemos concluir que existe uma vizinhança aberta de 0 tal que, para todo $t$ com $0 \leqslant t \leqslant 1$ e todo ponto $p$ nesta vizinhança, $\omega_{t}(p)$ é não-degenerada em $K_{0}^{\prime}=\mathbb{R}^{n} \oplus \mathbb{R}^{N} \oplus L_{0}$, ou seja, tem núcleo $K_{0}$. Ademais, para todo $t$ com $0 \leqslant t \leqslant 1$ e todo ponto $p$ nesta vizinhança, o subespaço $L_{0}$, sendo isotrópico tanto para $\omega_{0}$ como para $\omega(p)$, também é isotrópico para $\omega_{t}(p)$ e. como contém o núcleo de $\omega_{t}(p)$ e tem a dimensão correta dada pela equação (1.62), é multilagrangeano para $\omega_{t}(p)$, conforme o Teorema 1.6. Por outro lado, temos $d \omega_{0}=0$ (trivialmente) e $d \omega=0$ (por hipótese) e portanto uma versão apropriada do lema de Poincaré (veja o Apêndice) garante, em uma vizinhança aberta de 0 em $P$, a existência de uma $k$-forma $\alpha$ tal que $d \alpha=\omega_{0}-\omega$ e $\alpha^{b}(L)=0$. Seja agora $X_{t}$ o campo vetorial em $P$ dependente do tempo e a valores em $L_{0}{ }^{3}$ definido por

$$
\mathrm{i}_{X_{t}} \omega_{t}=\alpha
$$

e seja $F_{t} \equiv F_{(0, t)}$ seu fluxo a partir do instante 0 , mais uma vez definido em uma vizinhança aberta de $0 \mathrm{em} P$, para $0 \leqslant t \leqslant 1$. Então vale

$$
\begin{aligned}
\left.\frac{d}{d s}\right|_{s=t} F_{s}^{*} \omega_{s} & =F_{t}^{*}\left(\left.\frac{d}{d s}\right|_{s=t} \omega_{s}\right)+\left.\frac{d}{d s}\right|_{s=t} F_{s}^{*} \omega_{t} \\
& =F_{t}^{*}\left(\omega-\omega_{0}+L_{X_{t}} \omega_{t}\right) \\
& =F_{t}^{*}\left(\omega-\omega_{0}+d\left(i_{X_{t}} \omega_{t}\right)\right) \\
& =F_{t}^{*}\left(\omega-\omega_{0}+d \alpha\right) \\
& =0
\end{aligned}
$$

Portanto $F_{1}$ é a transformação de coordenadas desejada, já que $F_{1}^{*} \omega=F_{1}^{*} \omega_{1}=F_{0}^{*} \omega_{0}=\omega_{0}$. (Para informação adicional, veja [1].)

\footnotetext{
${ }^{3} \mathrm{E}$ neste ponto que entra crucialmente a propriedade de que $L_{0}$ é multilagrangeano e não apenas isotrópico ou mesmo maximal isotrópico (para $\omega_{t}(p)$, no caso).
} 
CAPÍtulo 3

\section{Estruturas poli- e multissimpléticas}

Neste capítulo, iniciamos um estudo mais específico das formas poli- e multissimpléticas. Nossa meta principal é providenciar, pelo menos para esta importante classe de formas poli- e multilagrangeanas, respectivamente, uma motivação mais sólida de sua definição. Acreditamos que isso seja necessário pois esta definição, baseada única e exclusivamente no critério da existência de um tipo especial de subespaço maximal isotrópico (chamado de subespaço poli- e multilagrangeano, respectivamente), constitui o ponto central do presente trabalho e porque já existem na literatura várias outras propostas para uma definição dos mesmos conceitos, o que requer uma comparação crítica.

Uma primeira tentativa de definir a noção de uma variedade multissimplética $(P, \omega)$ e provar um teorema de Darboux deve-se a Martin [19]. Neste trabalho, já apareceu a idéia de se exigir a existência de um subespaço isotrópico "grande", mas o significado matemático desta condição permaneceu nebuloso, inclusive porque o critério de ser "grande" foi formulado apenas como uma condição numérica de dimensão. Logo, notou-se que a fórmula de dimensão total resultante (que segue da fórmula (1.45) ou (1.46) pondo $\hat{n}=1$ e ker $\hat{\omega}=\{0\}$ ),

$$
\operatorname{dim} P=N+\left(\begin{array}{l}
N \\
k
\end{array}\right),
$$

é incompatível com o exemplo principal provindo da física, pois a dimensão dos espaços de multifase $(P, \omega)$ que aparecem no formalismo hamiltoniano covariante da teoria clássica dos campos é dada por uma fórmula diferente (que segue da fórmula (1.61) ou (1.62) pondo $k=n, r=2$ e $\operatorname{ker} \omega=\{0\})$,

$$
\operatorname{dim} P=(N+1)(n+1),
$$

obtida por uma simples contagem de coordenadas locais: na notação já empregada na introdução, temos $n$ coordenadas espaço-temporais $x^{\mu}, N$ variáveis posição $q^{i}, n N$ variáveis multimomento $p_{i}^{\mu}$ e uma variável energia $p$. Assim, e tendo em vista que o termo "multissimplético" já está ocupado na literatura pelo menos desde o início dos anos 1970 [13-15], 
parece-nos inadequado utilizar este mesmo termo para a estrutura estudada por Martin em [19], e portanto nos propomos substituí-lo pelo termo "polilagrangeano", convenção que adotamos neste trabalho.

Em artigos posteriores tais como [3] e [17], a necessidade de modificar e estender o formalismo através da inclusão de restrições de horizontalidade já foi claramente percebida, mas não existe até a presente data nenhum tratamento abrangente com demonstrações completas e corretas.

Em outros trabalhos, observa-se uma ênfase em questões pouco relevantes. Um exemplo que discutiremos com maiores detalhes são as hipóteses envolvendo condições sobre a algebra simétrica formada por potências exteriores de um multipleto de 2-formas adotadas em [11]: pretendemos demonstrar que estas, além de aparentemente não permitirem nenhuma extensão natural do caso polissimplético para o caso multissimplético, são ao mesmo tempo insufficientes e supérfluas para alcançar o objetivo (que é a formulãção e demonstração de uma versão do teorema de Darboux). Não obstante estes defeitos, o trabalho [11] tem sido importante para o desenvolvimento da nossa abordagem.

Com respeito à relação entre formas polissimpléticas e multissimpléticas, é interessante notar que as últimas são quase que por completo determinadas pelas primeiras, através do que chamamos de símbolo, pois este é uma forma polissimplética e, como veremos, de núcleo no máximo unidimensional. O que parametriza este núcleo é uma variável que na teoria clássica dos campos é relacionada com a energia.

\subsection{Espaços vetoriais polissimpléticos}

Conforme a Definição 1.1, uma 2-forma $\hat{\omega}$ em um espaço vetorial $V$ a valores em outro espaço vetorial $\hat{T}$, de dimensão $\hat{n}$, é dita polissimplética de posto $N$ se existir um subespaço polilagrangeano $L$ de $V$ de codimensão $N$, caracterizado pela propriedade de que

$$
\hat{\omega}^{b}(L)=L^{\perp} \otimes \hat{T} .
$$

Neste caso, fica óbvio que, de acordo com a fórmula (1.45) ou (1.46),

$$
\operatorname{dim} L=\operatorname{dim} \operatorname{ker} \hat{\omega}+\hat{n} N
$$

e portanto

$$
\operatorname{dim} V=\operatorname{dim} \operatorname{ker} \hat{\omega}+(\hat{n}+1) N .
$$

Em particular, a dimensão de um espaço vetorial munido de uma forma polissimplética nãodegenerada a valores em um espaço vetorial de dimensão $\hat{n}$ deve ser um múltiplo de $\hat{n}+1$, 
o que generaliza uma propriedade bem conhecida de formas simpléticas, que constituem o caso especial $\hat{n}=1$ (formas comuns). ${ }^{1}$ Mais geralmente, temos

$$
\operatorname{rk}(\hat{\omega})=N=\frac{1}{\hat{n}+1} \operatorname{dim} \operatorname{supp} \hat{\omega},
$$

configurando mais um exemplo do fenômeno já mencionado no primeiro capítulo, conforme o qual pode ser conveniente usar o termo "posto" de uma forma para uma expressão numericamente diferente da dimensão do seu suporte mas unicamente determinada por esta.

Quando $\hat{n} \geqslant 2$, temos o seguinte critério para decidir se uma 2-forma é polissimplética:

Proposição 3.1 Sejam $V$ e $\hat{T}$ espaços vetoriais de dimensão finita, com $\hat{n} \equiv \operatorname{dim} \hat{T} \geqslant 2$, e seja $\hat{\omega}$ uma 2-forma em $V$ a valores em $\hat{T}$. Considere o subespaço $L$ de $V$ gerado pelos núcleos de todas as projeçôes $\hat{\omega}_{\hat{t}^{*}}\left(\hat{t}^{*} \in \hat{T}^{*} \backslash\{0\}\right)$ de $\hat{\omega}$ :

$$
L=\sum_{\hat{t}^{*} \in \hat{T}^{*} \backslash\{0\}} \operatorname{ker} \hat{\omega}_{\hat{t}^{*}} .
$$

Para a forma $\hat{\omega}$ ser polissimplética e $L$ ser o subespaço polilagrangeano pertinente, as seguintes condiçôes são necessárias e suficientes:

- L é isotrópico;

- $\operatorname{dim} L=(\operatorname{dim} \operatorname{ker} \hat{\omega}+\hat{n} \operatorname{dim} V) /(\hat{n}+1)$.

DemonstraÇÃo. Trata-se de um corolário trivial dos Teoremas 1.3 e 1.4 .

Para formular o teorema de Darboux (algébrico) no contexto específico de formas polissimpléticas, começamos por adaptar a Definição 1.5 a este contexto: explicitamente, uma base de Darboux polissimplética ou base canônica polissimplética é uma base $\left\{e_{i}, e_{a}^{j} \mid 1 \leqslant a \leqslant \hat{n}, 1 \leqslant i, j \leqslant N\right\}$ de um subespaço de $V$ complementar a ker $\hat{\omega}$, com base dual $\left\{e^{i}, e_{j}^{a} \mid 1 \leqslant a \leqslant \hat{n}, 1 \leqslant i, j \leqslant N\right\}$ do subespaço supp $\hat{\omega}=(\operatorname{ker} \hat{\omega})^{\perp}$ de $V^{*}$, em conjunto com uma base $\left\{\hat{e}_{a} \mid 1 \leqslant a \leqslant \hat{n}\right\}$ de $\hat{T}$, com base dual $\left\{\hat{e}^{a} \mid 1 \leqslant a \leqslant \hat{n}\right\}$ de $\hat{T}^{*}$, tal que

$$
\hat{\omega}=\left(e_{i}^{a} \wedge e^{i}\right) \otimes \hat{e}_{a},
$$

ou em termos das formas projetadas $\omega^{a}$ definidas conforme as equações (1.29) e (1.30),

$$
\omega^{a}=e_{i}^{a} \wedge e^{i} \quad(1 \leqslant a \leqslant \hat{n}) .
$$

\footnotetext{
${ }^{1}$ Note que para $\hat{n}=1$, a definição de uma forma polissimplética não-degenerada se reduz à de uma forma simplética, pois a existência de um subespaço lagrangeano $L$ é um teorema, neste caso. A principal diferença entre este caso e os casos em que $\hat{n}>1$ é que aqui, $L$ não é único.
} 
Obviamente, uma 2-forma $\hat{\omega} \in \bigwedge^{2} V^{*} \otimes \hat{T}$ que admite uma base canônica deste tipo é uma forma polissimplética, sendo que a soma direta $L$ do núcleo de $\hat{\omega}$ com o subespaço $(\hat{n} N)$ dimensional gerado pelos vetores $e_{a}^{i}(1 \leqslant a \leqslant \hat{n}, 1 \leqslant i \leqslant N)$ é um subespaço polilagrangeano de $V$. É interessante notar que, por outro lado, a soma direta do núcleo de $\hat{\omega}$ com o subespaço $N$-dimensional gerado pelos vetores $e_{i}(1 \leqslant i \leqslant N)$ é um subespaço maximal isotrópico de $V$ que não é polilagrangeano.

Reciprocamente, como corolário do Teorema 1.9, temos

\section{Teorema 3.1 (Teorema de Darboux para espaços vetoriais polissimpléticos)}

Todo espaço polissimplético possui uma base canônica polissimplética.

Apesar deste teorema já ter sido provado no primeiro capítulo, queremos ainda apresentar uma demonstração alternativa que é estritamente paralela à demonstração do teorema correspondente para formas simpléticas e para formas bilineares simétricas, usando um processo indutivo do tipo "Gram-Schmidt" que inclusive serve para construir, indutiva e explicitamente, um subespaço isotrópico complementar ao subespaço polilagrangeano $L$ (veja, por exemplo, [1, Prop. 3.1.2, pp. 162-164]).

DemonstraçÃo. Denotando o subespaço polilagrangeano de $V$ por $L$, como sempre, e fixando um subespaço $L^{\prime}$ de $L$ complementar a ker $\hat{\omega}$, procedemos por indução em $N=\operatorname{dim} V-\operatorname{dim} L$. Para tanto, escolhemos arbitrariamente uma 1-forma $e^{N} \in L^{\perp} \backslash\{0\}$ e um vetor $e_{N} \in V \backslash L$ tal que

$$
\left\langle e^{N}, e_{N}\right\rangle=1 \text {, }
$$

e usando a propriedade (3.1), definimos os vetores $e_{a}^{N} \in L^{\prime}(1 \leqslant a \leqslant \hat{n})$ por $^{2}$

$$
\hat{\omega}^{b}\left(e_{a}^{N}\right)=-e^{N} \otimes \hat{e}_{a},
$$

ou em termos das formas projetadas

$$
\left(\omega^{b}\right)^{b}\left(e_{a}^{N}\right)=-\delta_{a}^{b} e^{N}
$$

e as 1 -formas $e_{N}^{a} \in V^{*}(1 \leqslant a \leqslant \hat{n})$ por

$$
e_{N}^{a}=\left(\omega^{a}\right)^{b}\left(e_{N}\right)
$$

Assim temos

$$
\left\langle e^{N}, e_{1}^{N}\right\rangle=-\omega^{1}\left(e_{1}^{N}, e_{1}^{N}\right)=0 \quad \ldots\left\langle e^{N}, e_{\hat{n}}^{N}\right\rangle=-\omega^{\hat{n}}\left(e_{\hat{n}}^{N}, e_{\hat{n}}^{N}\right)=0
$$

$\mathrm{e}$

$$
\omega^{b}\left(e_{N}, e_{a}^{N}\right)=\delta_{a}^{b}
$$

\footnotetext{
${ }^{2}$ Note que isto determina os $e_{a}^{N}$ unicamente em termos de $e^{N}$.
} 
Usando que $L$ é isotrópico relativo a $\hat{\omega}$ e portanto a cada uma das formas projetadas, temos ainda

$$
\omega^{c}\left(e_{a}^{N}, e_{b}^{N}\right)=0 .
$$

Isto implica que podemos decompor o espaço $V$ na soma direta

$$
V=V_{N} \oplus V_{N}^{\hat{\omega}}
$$

do subespaço $V_{N}$ gerado pelos vetores $e_{N}$ e $e_{a}^{N}(1 \leqslant a \leqslant \hat{n})$, de dimensão $\hat{n}+1$, e seu complemento ortogonal simultâneo $V_{N}^{\hat{\omega}}$, que é a intersecção dos $\hat{n}$ diferentes complementos ortogonais de $V_{N}$ relativos a cada uma das formas projetadas. Ainda mais, a fórmula

$$
P_{N}^{\hat{\omega}}(v)=v-\left\langle e^{N}, v\right\rangle e_{N}-\omega^{a}\left(e_{N}, v\right) e_{a}^{N}
$$

provê uma definição explícita do projetor $P_{N}^{\hat{\omega}}$ sobre $V_{N}^{\hat{\omega}}$ ao longo de $V_{N}$. [Para provar esta afirmação, note que

$$
\begin{aligned}
& P_{N}^{\hat{\omega}}\left(e_{N}\right)=e_{N}-\left\langle e^{N}, e_{N}\right\rangle e_{N}-\omega^{a}\left(e_{N}, e_{N}\right) e_{a}^{N}=0, \\
& P_{N}^{\hat{\omega}}\left(e_{a}^{N}\right)=e_{a}^{N}-\left\langle e^{N}, e_{a}^{N}\right\rangle e_{N}-\omega^{b}\left(e_{N}, e_{a}^{N}\right) e_{b}^{N}=0,
\end{aligned}
$$

e que, para todo $v \in V$,

$$
\begin{aligned}
& \omega^{c}\left(P_{N}^{\hat{\omega}}(v), e_{N}\right)=\omega^{c}\left(v, e_{N}\right)-\left\langle e^{N}, v\right\rangle \omega^{c}\left(e_{N}, e_{N}\right)-\omega^{b}\left(e_{N}, v\right) \omega^{c}\left(e_{b}^{N}, e_{N}\right)=0, \\
& \omega^{c}\left(P_{N}^{\hat{\omega}}(v), e_{a}^{N}\right)=\omega^{c}\left(v, e_{a}^{N}\right)-\left\langle e^{N}, v\right\rangle \omega^{c}\left(e_{N}, e_{a}^{N}\right)-\omega^{b}\left(e_{N}, v\right) \omega^{c}\left(e_{b}^{N}, e_{a}^{N}\right)=0,
\end{aligned}
$$

o que mostra que $P_{N}^{\hat{\omega}}$ se anula em $V_{N}$ e mapea o espaço inteiro $V$ no subespaço $V_{N}^{\hat{\omega}}$. Usando novamente a definição de $P_{N}^{\hat{\omega}}$, concluímos ainda que ker $P_{N}^{\hat{\omega}}=V_{N}$, pois um argumento de independência linear mostra que $P_{N}^{\hat{\omega}}$ não pode se anular em nenhum vetor que não pertença ao subespaço gerado pelos vetores $e_{N}$ e $e_{a}^{N}(1 \leqslant a \leqslant \hat{n})$, e que $P_{N}^{\hat{\omega}}$ é a identidade em $V_{N}^{\hat{\omega}}$, pois usando que $\left\langle e^{N}, v\right\rangle=-\omega^{a}\left(e_{a}^{N}, v\right)$ para qualquer valor fixo de $a$ (sem soma), vemos que a 1 -forma $e^{N}$ se anula em $V_{N}^{\hat{\omega}}$. Logo, $P_{N}^{\hat{\omega}}$ é um projetor (i.e., vale $\left.\left(P_{N}^{\hat{\omega}}\right)^{2}=P_{N}^{\hat{\omega}}\right)$ com núcleo $V_{N}$ e imagem $V_{N}^{\hat{\omega}}$.] Passando para o dual, esta decomposição direta de $V$ induz uma correspondente decomposição direta

$$
V^{*}=V_{N}^{*} \oplus\left(V_{N}^{\hat{\omega}}\right)^{*}
$$

de $V^{*}$, onde o dual de cada subespaço é naturalmente identificado com o aniquilador do outro:

$$
V_{N}^{*}=\left(V_{N}^{\hat{\omega}}\right)^{\perp}, \quad\left(V_{N}^{\hat{\omega}}\right)^{*}=V_{N}^{\perp} .
$$

Notando que sob estas decomposições $L$ se torna a soma direta da intersecção $L \cap V_{N}^{\hat{\omega}}$ e do subespaço gerado pelos vetores $e_{a}^{N}(1 \leqslant a \leqslant \hat{n})$ enquanto $L^{\perp}$ se torna a soma direta da intersecção $L^{\perp} \cap V_{N}^{\perp}$ e do subespaço unidimensional gerado pela 1-forma $e^{N}$. Assim, fica 
claro que podemos repetir o mesmo processo para a restrição da forma $\hat{\omega}$ ao subespaço $V_{N}^{\hat{\omega}}$, que é uma forma polissimplética de posto $N-1$ (como pode ser visto, por exemplo, por uma análise dos critérios da Proposição 3.1), de modo que após $N$ repetições do mesmo processo, chegamos à conclusão do teorema.

Concluiremos esta seção com uma digressão sobre 2-formas a valores vetoriais em geral, objetivando assim entender melhor a simplicidade e utilidade do conceito de forma polissimplética adotado nesta tese, em comparação com outras definições que antecederam a nossa. Certamente, encontrar o caminho certo nos meandros das noções possíveis foi a parte mais difícil do trabalho.

Suponha então que $\hat{\omega} \in \bigwedge^{2} V^{*} \otimes \hat{T}$ é uma 2-forma qualquer em $V$ a valores em $\hat{T}$. Inicialmente, lembramos que conforme a convenção adotada neste trabalho (veja, por exemplo, a equação (3.4)), o posto de uma 2-forma $\omega$ comum (i.e., a valores reais) é igual à metade da dimensão do seu suporte. Assim, temos para todo $\hat{t}^{*} \in \hat{T}^{*}$,

$$
\operatorname{rk}\left(\omega_{\hat{t}^{*}}\right)=\frac{1}{2} \operatorname{dim} \operatorname{supp} \omega_{\hat{t}^{*}} .
$$

Agora notamos que a aplicação linear

$$
\begin{aligned}
& \hat{T}^{*} \longrightarrow \wedge^{2} V^{*} \\
& \hat{t}^{*} \longmapsto \omega_{\hat{t}^{*}}
\end{aligned}
$$

induz, para todo inteiro $k \geqslant 1$ e de forma canônica, uma aplicação linear

$$
\begin{aligned}
& \bigvee^{k} \hat{T}^{*} \longrightarrow \bigwedge^{2 k} V^{*} \\
& P \longmapsto P(\hat{\omega})
\end{aligned}
$$

onde identificamos o espaço $\bigvee^{k} \hat{T}^{*}$ dos $k$-tensores covariantes simétricos em $\hat{T}$ com o espaço dos polinômios homogêneos $P$ de grau $k$ em $\hat{T}$. Explicitamente, em termos de uma base $\left\{\hat{e}_{a} \mid 1 \leqslant a \leqslant \hat{n}\right\}$ de $\hat{T}$, com base dual $\left\{\hat{e}^{a} \mid 1 \leqslant a \leqslant \hat{n}\right\}$ de $\hat{T}^{*}$, temos

$$
P=P_{a_{1} \ldots a_{k}} \hat{e}^{a_{1}} \vee \ldots \vee \hat{e}^{a_{k}} \Longrightarrow P(\hat{\omega})=P_{a_{1} \ldots a_{k}} \omega^{a_{1}} \wedge \ldots \wedge \omega^{a_{k}} .
$$

Isso nos permite introduzir a seguinte terminologia:

Definição 3.1 Sejam $V$ e $\hat{T}$ espaços vetoriais de dimensão finita e seja $\hat{\omega}$ uma 2-forma em $V$ a valores em $\hat{T}$. Dizemos que $\hat{\omega}$ tem posto constante $N$ se $\operatorname{rk}\left(\omega_{\hat{t}^{*}}\right)=N$ para todo $\hat{t}^{*} \in \hat{T}^{*} \backslash\{0\}$ e dizemos que $\hat{\omega}$ tem posto uniforme $N$ se a aplicação linear (3.10) for injetora para $k=N$ e identicamente zero para $k=N+1$. 
Usando multi-índices $\alpha=\left(\alpha_{1}, \ldots, \alpha_{\hat{n}}\right) \in \mathbb{N}^{\hat{n}}$, pomos

$$
\hat{e}^{\alpha}=\left(\hat{e}^{1}\right)^{\alpha_{1}} \vee \ldots \vee\left(\hat{e}^{\hat{n}}\right)^{\alpha_{\hat{n}}} \quad \text { onde } \quad\left(\hat{e}^{a}\right)^{\alpha_{a}}=\hat{e}^{a} \vee \ldots \vee \hat{e}^{a} \quad\left(\alpha_{a} \text { vezes }\right)
$$

e

$$
\omega^{\alpha}=\left(\omega^{1}\right)^{\alpha_{1}} \wedge \ldots \wedge\left(\omega^{\hat{n}}\right)^{\alpha_{\hat{n}}} \quad \text { onde } \quad\left(\omega^{a}\right)^{\alpha_{a}}=\omega^{a} \wedge \ldots \wedge \omega^{a} \quad\left(\alpha_{a} \text { vezes }\right)
$$

para reescrever a equação (3.10) na forma

$$
P=\sum_{|\alpha|=k} P_{\alpha} \hat{e}^{\alpha} \Longrightarrow P(\hat{\omega})=\sum_{|\alpha|=k} P_{\alpha} \omega^{\alpha} .
$$

Como $\left\{\hat{e}^{\alpha}|| \alpha \mid=k\right\}$ é uma base de $\bigvee^{k} \hat{T}^{*}$, o requerimento de que $\hat{\omega}$ tenha posto uniforme $N$ pode ser reformulado como segue:

$$
\begin{gathered}
\left\{\omega^{\alpha}|| \alpha \mid=N\right\} \text { é linearmente independente } \\
\omega^{\alpha}=0 \text { para }|\alpha|=N+1
\end{gathered}
$$

É nesta forma que a condição de posto uniforme aparece como parte da definição de forma polissimplética em [11].

Para um melhor entendimento das condições de posto introduzidas acima, notamos primeiro que ambas generalizam a noção de posto de formas comuns. De fato, quando $\hat{n}=1$, ou seja, para uma 2-forma $\omega$ comum de posto $N$ em $V$, podemos escolher uma base canônica $\left\{e_{1}, \ldots, e_{N}, f^{1}, \ldots, f^{N}\right\}$ de um subespaço de $V$ complementar ao núcleo de $\omega$, com base dual $\left\{e^{1}, \ldots, e^{N}, f_{1}, \ldots, f_{N}\right\}$ do subespaço supp $\omega$ de $V^{*}$, para concluir que $\omega=e^{i} \wedge f_{i}$ e portanto

$$
\omega^{N}= \pm e^{1} \wedge \ldots \wedge e^{N} \wedge f_{1} \wedge \ldots \wedge f_{N} \neq 0 \quad, \quad \omega^{N+1}=0 .
$$

Em outras palavras, o posto de $\omega$ é caracterizado como sendo aquele inteiro positivo $N$ para o qual vale $\omega^{N} \neq 0$ mas $\omega^{N+1}=0$. A partir desta observação, segue que, no caso geral considerado acima, a condição de posto uniforme implica a de posto constante pois ela de fato garante que para todo $\hat{t}^{*} \in \hat{T}^{*} \backslash\{0\}$ vale $\omega_{\hat{t}^{*}}^{N} \neq 0$ e $\omega_{\hat{t}^{*}}^{N+1}=0$, ou seja, $\operatorname{rk}\left(\omega_{\hat{t}^{*}}\right)=N$. Porém a conclusão recíproca não é válida, como mostra o seguinte

Exemplo 3.1 $(\hat{n}=2, N=2, \operatorname{dim} V=4, \operatorname{ker} \hat{\omega}=\{0\})$ :

Seja $V=\mathbb{R}^{4}, \hat{T}=\mathbb{R}^{2}$ e considere a 2-forma $\hat{\omega}$ constituída pelas seguintes duas 2-formas comuns:

$$
\omega^{1}=d x \wedge d y+d u \wedge d v \quad, \quad \omega^{2}=d x \wedge d u-d y \wedge d v .
$$

Então para $\hat{t}^{*}=\hat{t}_{a}^{*} \hat{e}^{a} \in\left(\mathbb{R}^{2}\right)^{*}$, vale

$$
\omega_{\hat{t}^{*}}=\hat{t}_{a}^{*} \omega^{a}=d x \wedge\left(\hat{t}_{1}^{*} d y+\hat{t}_{2}^{*} d u\right)+d v \wedge\left(\hat{t}_{2}^{*} d y-\hat{t}_{1}^{*} d u\right) .
$$


Assim temos, para todo $\hat{t}^{*} \neq 0$

$$
\left(\omega_{\hat{t}^{*}}\right)^{2} \equiv \omega_{\hat{t}^{*}} \wedge \omega_{\hat{t}^{*}}=\left(\left(\hat{t}_{1}^{*}\right)^{2}+\left(\hat{t}_{2}^{*}\right)^{2}\right) d x \wedge d y \wedge d u \wedge d v \neq 0,
$$

enquanto que, por estarmos em um espaço quadridimensional,

$$
\left(\omega_{\hat{t}^{*}}\right)^{3} \equiv \omega_{\hat{t}^{*}} \wedge \omega_{\hat{t}^{*}} \wedge \omega_{\hat{t}^{*}}=0
$$

garantindo que $\hat{\omega}$ tem posto constante 2 . Porém $\hat{\omega}$ não tem posto uniforme 2 , uma vez que

$$
\omega^{1} \wedge \omega^{2}=0 .
$$

Por outro lado, formas polissimpléticas tem posto uniforme:

Proposição 3.2 Sejam $V$ e $\hat{T}$ espaços vetoriais de dimensão finita e seja $\hat{\omega}$ uma 2-forma polissimplética em $V$ a valores em $\hat{T}$ de posto $N$. Então $\hat{\omega}$ tem posto uniforme $N$.

DemonstraÇÃo. Introduzindo uma base canônica polissimplética em que $\hat{\omega}$ assume a forma dada pelas equações (3.5) e (3.6), suponha que $\alpha=\left(\alpha_{1}, \ldots, \alpha_{\hat{n}}\right) \in \mathbb{N}^{\hat{n}}$ é um multiíndice de grau $k$ (i.e., tal que $|\alpha|=\alpha_{1}+\ldots+\alpha_{\hat{n}}=k$ ) e considere a forma

$$
\begin{aligned}
\omega^{\alpha}= \pm( & \left.\left(e^{i_{1}^{1}} \wedge \ldots \wedge e^{i_{\alpha_{1}}^{1}}\right) \wedge \ldots \wedge\left(e^{i_{1}^{\hat{n}}} \wedge \ldots \wedge e^{i_{\alpha_{\hat{n}}^{\hat{n}}}^{\hat{n}}}\right)\right) \\
& \wedge\left(e_{i_{1}^{1}}^{1} \wedge \ldots \wedge e_{i_{\alpha_{1}}^{1}}^{1}\right) \wedge \ldots \wedge\left(e_{i_{1}^{\hat{n}}}^{\hat{n}} \wedge \ldots \wedge e_{i_{\alpha_{\hat{n}}}^{\hat{n}}}^{\hat{n}}\right) .
\end{aligned}
$$

Obviamente qualquer tal forma se anula para $k=N+1$ porque contêm um produto exterior de $(N+1) 1$-formas $e^{i}$ pertencendo a um subespaço $N$-dimensional. Por outro lado, todas estas formas são linearmente independentes se $k=N$ porque neste caso $\omega^{\alpha}$ contém o produto exterior $e^{1} \wedge \ldots \wedge e^{N}$ multiplicado pelos produto exterior de $\alpha_{1}$ 1-formas do tipo $e_{i}^{1} \operatorname{com} \ldots$ com $\alpha_{\hat{n}} 1$-formas do tipo $e_{i}^{\hat{n}}$; assim $\omega^{\alpha}$ e $\omega^{\beta}$ pertencem a diferentes subespaços de $\bigwedge^{2 N} V^{*}$ sempre que $\alpha \neq \beta$.

Como veremos logo adiante, a afirmação recíproca está longe de ser verdade. De fato, se ela fosse verdadeira, então para $\hat{n} \geqslant 2$ devería ser possível construir o subespaço polilagrangeano como a soma dos núcleos das formas projetadas, conforme exigido pela Proposição 3.1. Logo, devería ser possível mostrar que o subespaço definido como a soma destes núcleos é isotrópico. E de fato, como resultado parcial nesta direção, temos a seguinte

Proposição 3.3 Sejam $V$ e $\hat{T}$ espaços vetoriais de dimensão finita e seja $\hat{\omega}$ uma 2-forma em $V$ a valores em $\hat{T}$ de posto uniforme $N$. Então, para quaisquer $\hat{t}_{1}^{*}, \hat{t}_{2}^{*} \in \hat{T}^{*} \backslash\{0\}$, o núcleo de $\omega_{\hat{t}_{1}^{*}}$ é isotrópico relativo a $\omega_{\hat{t}_{2}^{*}}$. 
DemonstraÇÃO. Dados $u, v \in \operatorname{ker} \omega_{\hat{t}_{1}^{*}}$, temos

e portanto

$$
\mathrm{i}_{u} \omega_{\hat{t}_{1}^{*}}^{N}=N \mathrm{i}_{u} \omega_{\hat{t}_{1}^{*}} \wedge \omega_{\hat{t}_{1}^{*}}^{N-1}=0 \quad, \quad \mathrm{i}_{v} \omega_{\hat{t}_{1}^{*}}^{N}=N \mathrm{i}_{v} \omega_{\hat{t}_{1}^{*}} \wedge \omega_{\hat{t}_{1}^{*}}^{N-1}=0,
$$

$$
\omega_{\hat{t}_{2}^{*}}(u, v) \omega_{\hat{t}_{1}^{*}}^{N}=\mathrm{i}_{u} \mathrm{i}_{v}\left(\omega_{\hat{t}_{2}^{*}} \wedge \omega_{\hat{t}_{1}^{*}}^{N}\right)=0 .
$$

Usando que $\omega_{\hat{t}_{1}^{*}}^{N} \neq 0$, segue que $\omega_{\hat{t}_{2}^{*}}(u, v)=0$.

No entanto a condição de isotropia do subespaço definido como a soma dos núcleos de todas as formas projetadas, que equivale à condição (mais forte) de que para quaisquer $\hat{t}_{1}^{*}, \hat{t}_{2}^{*}, \hat{t}_{3}^{*} \in \hat{T}^{*} \backslash\{0\}$, ker $\omega_{\hat{t}_{1}^{*}}$ e ker $\omega_{\hat{t}_{1}^{*}}$ são ortogonais sob $\omega_{\hat{t}_{3}^{*}}$, i.e., vale

$$
\omega_{\hat{t}_{3}^{*}}\left(u_{1}, u_{2}\right)=0 \quad \text { para } u_{1} \in \operatorname{ker} \omega_{\hat{t}_{1}^{*}} \text { e } u_{2} \in \operatorname{ker} \omega_{\hat{t}_{2}^{*}},
$$

não pode ser deduzida da condição de posto uniforme. Um belo contraexemplo é obtido quando escolhemos $V$ e $\hat{T}$ como sendo o mesmo espaço, supondo que este seja uma álgebra de Lie $\mathfrak{g}$ e definindo $\hat{\omega}$ como sendo o comutador em $\mathfrak{g}$. Então para $\hat{t}^{*} \in \mathfrak{g}^{*}$, o núcleo ker $\omega_{\hat{t}^{*}}$ e o suporte $\operatorname{supp} \omega_{\hat{t}^{*}}$ da forma projetada $\omega_{\hat{t}^{*}}$ são, respectivamente, a álgebra de isotropia de $\hat{t}^{*}$ e o espaço tangente à órbita coadjunta que passa por $\hat{t}^{*}$. Existe uma única álgebra semisimples para a qual $\hat{\omega}$ tem posto constante, uma vez que esta condição implica que todas as órbitas coadjuntas, exceto a órbita trivial $\{0\}$, devem ter a mesma dimensão: esta é a álgebra do tipo $A_{1}$, quer dizer, $\mathbb{R}^{3}$ equipado com o produto vetorial $\times$.

Exemplo 3.2 $(\hat{n}=3, N=1, \operatorname{dim} V=3, \operatorname{ker} \hat{\omega}=\{0\})$ :

Seja $V=\hat{T}=\mathbb{R}^{3}$ e considere a 2 -forma $\hat{\omega}$ constituída pelas seguintes três 2 -formas comuns:

$$
\omega^{1}=d y \wedge d z \quad, \quad \omega^{2}=d z \wedge d x \quad, \quad \omega^{3}=d x \wedge d y .
$$

Então para $\hat{t}^{*}=\hat{t}_{a}^{*} \hat{e}^{a} \in\left(\mathbb{R}^{3}\right)^{*}$, vale

$$
\omega_{\hat{t}^{*}}=\hat{t}_{a}^{*} \omega^{a}=\hat{t}_{1}^{*} d y \wedge d z+\hat{t}_{2}^{*} d z \wedge d x+\hat{t}_{3}^{*} d x \wedge d y .
$$

Obviamente, $\omega^{1}, \omega^{2}$ e $\omega^{3}$ são linearmente independentes e portanto $\hat{\omega}$ possui posto uniforme 1 , uma vez que não existe nenhuma 4-forma não nula em um espaço tridimensional. Por outro lado, temos

$$
\operatorname{ker} \omega_{\hat{t}^{*}}=\left\langle\hat{t}_{1}^{*} \frac{\partial}{\partial x}+\hat{t}_{2}^{*} \frac{\partial}{\partial y}+\hat{t}_{3}^{*} \frac{\partial}{\partial z}\right\rangle,
$$

Portanto a intersecção dos três núcleos ker $\omega^{1}$, ker $\omega^{2}$ e $\operatorname{ker} \omega^{3}$ é $\{0\}$ (i.e., $\hat{\omega}$ é nãodegenerada). Contudo ker $\omega^{1}$ e ker $\omega^{2}$ são ortogonais sob $\omega^{1}$ e sob $\omega^{2}$ mas não sob $\omega^{3}$. Agora se existisse um subespaço polilagrangeano então ele deveria coincidir com a soma dos núcleos de todas as formas projetadas, mas este não é isotrópico. Logo $\hat{\omega}$ não possui subespaço polilagrangeano. 
Finalmente, observamos que mesmo se a soma dos núcleos de todas as formas projetadas for um subespaço isotrópico relativo a $\hat{\omega}$, ainda assim este pode não ser um subespaço polilagrangeano, como mostra o seguinte

Exemplo 3.3 $(\hat{n}=2, N=2, \operatorname{dim} V=5, \operatorname{ker} \hat{\omega}=\{0\})$ :

Seja $V=\mathbb{R}^{5}, \hat{T}=\mathbb{R}^{2}$ e considere a 2-forma $\hat{\omega}$ constituída pelas seguintes duas 2-formas comuns:

$$
\omega^{1}=d x^{1} \wedge d x^{4}+d x^{2} \wedge d x^{3} \quad, \quad \omega^{2}=d x^{1} \wedge d x^{3}-d x^{2} \wedge d x^{5} .
$$

Então para $\hat{t}^{*}=\hat{t}_{a}^{*} \hat{e}^{a} \in\left(\mathbb{R}^{2}\right)^{*}$, vale

$$
\omega_{\hat{t}^{*}}=\hat{t}_{a}^{*} \omega^{a}=d x^{1} \wedge\left(\hat{t}_{1}^{*} d x^{4}+\hat{t}_{2}^{*} d x^{3}\right)+d x^{2} \wedge\left(\hat{t}_{1}^{*} d x^{3}-\hat{t}_{2}^{*} d x^{5}\right) .
$$

Obviamente $\omega^{1}, \omega^{2}$ e as formas

$$
\begin{gathered}
\left(\omega^{1}\right)^{2} \equiv \omega^{1} \wedge \omega^{1}=2 d x^{1} \wedge d x^{2} \wedge d x^{3} \wedge d x^{4} \\
\omega^{1} \wedge \omega^{2}=d x^{1} \wedge d x^{2} \wedge d x^{4} \wedge d x^{5} \\
\left(\omega^{2}\right)^{2} \equiv \omega^{2} \wedge \omega^{2}=2 d x^{1} \wedge d x^{2} \wedge d x^{3} \wedge d x^{5}
\end{gathered}
$$

são linearmente independentes e portanto $\hat{\omega}$ tem posto uniforme 2 , uma vez que não existe nenhuma 6 -forma não nula em um espaço 5 -dimensional. Por outro lado, temos

$$
\operatorname{ker} \omega_{\hat{t}^{*}}=\left\langle\hat{t}_{1}^{*} \hat{t}_{2}^{*} \frac{\partial}{\partial x^{3}}-\left(\hat{t}_{2}^{*}\right)^{2} \frac{\partial}{\partial x^{4}}+\left(\hat{t}_{1}^{*}\right)^{2} \frac{\partial}{\partial x^{5}}\right\rangle .
$$

A intersecção dos dois núcleos ker $\omega^{1}$ e ker $\omega^{2}$ é $\{0\}$ (i.e., $\hat{\omega}$ é não-degenerada). Note que a soma (direta) destes é o subespaço bidimensional de $V$, diga $L^{\prime}$, gerado por $\partial / \partial x^{4}$ e $\partial / \partial x^{5}$, enquanto que o subespaço $L$ gerado por todos os núcleos ker $\omega_{\hat{t}^{*}}\left(\hat{t}^{*} \in \hat{T}^{*} \backslash\{0\}\right)$ é o subespaço tridimensional de $V$ gerado por $\partial / \partial x^{i}$ com $i=3,4,5$, e este é isotropico relativo a todas as formas $\omega_{\hat{t}^{*}}\left(\hat{t}^{*} \in \hat{T}^{*} \backslash\{0\}\right)$. Mais do que isso, como a sua codimensão é 2 , ele é maximal isotrópico relativo a todas as formas $\omega_{\hat{t}^{*}}\left(\hat{t}^{*} \in \hat{T}^{*} \backslash\{0\}\right)$. Agora se existisse um subespaço polilagrangeano então ele deveria coincidir com $L$ e também com $L^{\prime}$, mas estes dois não são iguais. Logo $\hat{\omega}$ não possui subespaço polilagrangeano.

Resumindo, os exemplos dados acima mostram que a hipótese da existência de um subespaço polilagrangeano é altamente não-trivial e bastante restritiva: parece que ela não pode ser substituída por nenhuma outra hipótese que não seja obviamente equivalente. Os exemplos também mostram a grande variedade de possibilidades para a "posição relativa" dos núcleos das diversas formas projetadas que prevalece quando tal subespaço não existe. Neste sentido, a definição adotada em [11] é altamente inconveniente, pois não faz nenhuma referência explícita a este subespaço e assim acaba camuflando o aspecto central da teoria. 
Por fim, queremos tecer algumas considerações acerca da relação entre o subespaço polilagrangeano, quando ele existe, e a classe mais geral dos subespaços maximais isotrópicos. Primeiro, ressaltamos que ao contrário de um subespaço polilagrangeano, espaços maximais isotrópicos sempre existem. Para provar isso, basta começar com um subespaço unidimensional qualquer $L_{1}$, que é automaticamente isotrópico, e construir uma cadeia de subespaços $L_{1} \subset L_{2} \subset \ldots$ onde $L_{p+1}$ é definido como a soma direta de $L_{p}$ e o subespaço unidimensional gerado por algum vetor não nulo de seu complemento 1-ortogonal $L_{p}^{\hat{\omega}, 1}$. Em algum momento, este processo deve terminar, o que significa que acabamos de construir um subespaço maximal isotrópico. No entanto, nada garante que subespaços maximais isotrópicos que resultam de cadeias diferentes tenham a mesma dimensão, nem que exista alguma cadeia levando a um subespaço maximal isotrópico de dimensão suficentemente alta para ser polilagrangeano: isso acontece apenas no caso especial de formas comuns $(\hat{n}=1)$, onde todos os subespaços maximais isotrópicos acabam tendo a mesma dimensão e onde as noções de subespaço polilagrangeano, ou simplesmente lagrangeano, neste caso, e de subespaço maximal isotrópico coincidem.

Outro ponto importante diz respeito à relação entre as noções de isotrópico e maximal isotrópico relativo a forma $\hat{\omega}$ e relativo às suas projeções. Primeiro, é óbvio que um subespaço de $V$ é isotrópico relativo a $\hat{\omega}$ se e somente se for isotrópico relativo a cada uma das formas projetadas $\omega_{\hat{t}^{*}}\left(\hat{t}^{*} \in T^{*} \backslash\{0\}\right)$ ou $\omega^{a}(1 \leqslant a \leqslant \hat{n})$. Contudo o mesmo não vale quando substituírmos a palavra "isotrópico" pela expressão "maximal isotrópico": um subespaço de $V$ que é maximal isotrópico relativo a cada uma das projeções de $\hat{\omega}$ certamente será maximal isotrópico relativo a $\hat{\omega}$, mas reciprocamente, ele pode muito bem ser maximal isotrópico relativo a $\hat{\omega}$ (e portanto isotrópico relativo a cada uma das projeções de $\hat{\omega}$ ) mas ainda assim deixar de ser maximal isotrópico relativo a algumas delas. E finalmente, um subespaço polilagrangeano de $V$ certamente será maximal isotrópico relativo a cada uma das projeções de $\hat{\omega}$ (isso segue da Proposição 3.1), mas reciprocamente, como vimos no último exemplo acima, ele pode ser até maximal isotrópico relativo a cada uma das projeções de $\hat{\omega}$ sem ser polilagrangeano. Essas afirmações ilustram a natureza especial do subespaço polilagrangeano, já no caso de 2 -formas a valores vetoriais.

\subsection{Espaços vetoriais multissimpléticos}

Conforme a Definição 1.2, uma $(n+1)$-forma $\omega$ em um espaço vetorial $W$ que é $(n-1)$ horizontal em relação a um subespaço $V$ de $W$ de codimensão $n$ em $W$ é dita multissimplética de posto $N$ se for não-degenerada e se existir um subespaço multilagrangeano $L$ de $V$ de codimensão $N$ em $V$ (e portanto de codimensão $N+n$ em $W$ ), caracterizado pela propriedade de que

$$
\omega_{V}^{b}(L)=\bigwedge_{1}^{n} L^{\perp} \equiv \bigwedge^{n} L^{\perp} \cap \bigwedge_{1}^{n} W^{*}
$$


Neste caso, fica óbvio que, de acordo com a fórmula (1.61) ou (1.62),

$$
\operatorname{dim} L=N n+1,
$$

e portanto

$$
\operatorname{dim} V=N(n+1)+1,
$$

e

$$
\operatorname{dim} W=(N+1)(n+1) .
$$

Mais uma vez, concluímos que a dimensão de um espaço vetorial munido de uma forma multissimplética deve ser um múltiplo do seu grau $n+1$, o que generaliza uma propriedade bem conhecida de formas simpléticas, que constituem o caso especial $n=1$ (formas comuns). ${ }^{3}$ Mais geralmente, temos

$$
\operatorname{rk}(\omega)=N=\frac{1}{n+1} \operatorname{dim} \operatorname{supp} \omega-1,
$$

configurando mais um exemplo do fenômeno já mencionado no primeiro capítulo, conforme o qual pode ser conveniente usar o termo "posto" de uma forma para uma expressão numericamente diferente da dimensão do seu suporte mas unicamente determinada por esta.

Dada uma $(n+1)$-forma $\omega$ em um espaço vetorial $W$ que é $(n-1)$-horizontal em relação a um subespaço $V$ de $W$ de codimensão $n$ em $W$, podemos lhe associar o seu símbolo que será então uma 2-forma em $W$ a valores no espaço $\hat{T}=\bigwedge^{n-1} T^{*}$, onde $T \cong W / V$. Note que o espaço $\hat{T}$ tem a mesma dimensão que o próprio espaço base $T: \hat{n}=n$. Ademais, sabemos que se $\omega$ for multissimplética, então $\hat{\omega}$ será polissimplética, sendo que o espaço polilagrangeano para $\hat{\omega}$ será o mesmo que o espaço multilagrangeano para $\omega$. Comparando as equações (3.14) e (3.2), concluímos que, neste caso,

$$
\operatorname{dim} \operatorname{ker} \hat{\omega}=1 \text {. }
$$

De fato, como por hipótese $\omega$ é não-degenerada, a aplicação linear

$$
\begin{aligned}
\omega_{V}^{b}: V & \longrightarrow \bigwedge_{1}^{n} W^{*} \\
v & \longmapsto \mathrm{i}_{v} \omega
\end{aligned}
$$

(veja a equação (1.53)) é injetora e ker $\hat{\omega}$ é exatamente a imagem inversa, sob $\omega_{V}^{b}$, do subespaço $\bigwedge_{0}^{n} W^{*} \cong \bigwedge^{n} T^{*}$, que é unidimensional.

Quando $n \geqslant 2$, estas considerações, em conjunto com a Proposição 3.1, levam ao seguinte critério para decidir se uma $(n+1)$-forma é multissimplética:

\footnotetext{
${ }^{3}$ Note que para $n=1$, a definição de uma forma multissimplética se reduz à de uma forma simplética, com duas condições adicionais que são supérfluas no sentido de serem trivialmente satisfeitas: a hipótese de 0-horizontalidade é vazia e a da existência de um subespaço lagrangeano $L$ é um teorema, neste caso. A principal diferença entre este caso e os casos em que $n>1$ é que aqui, $L$ não é único.
} 
Proposição 3.4 Sejam $W$ um espaço vetorial de dimensão finita e $V$ um subespaço fixo de $W$, com $n=\operatorname{dim}(W / V) \geqslant 2$, e seja $\omega$ uma $(n+1)$-forma não-degenerada em $W$ que é (n-1)-horizontal, com símbolo $\hat{\omega}$ que é uma 2 -forma em $W$ a valores no espaço $\hat{T}=\bigwedge^{n-1} T^{*}$, onde $T \cong W / V$. Considere o subespaço $L$ de $V$ gerado pelos núcleos de todas as projeções $\omega_{\hat{t}}\left(\hat{t} \in \hat{T}^{*} \backslash\{0\}=\bigwedge^{n-1} T \backslash\{0\}\right)$ de $\hat{\omega}:$

$$
L=\sum_{\hat{t} \in \hat{T}^{*} \backslash\{0\}} \operatorname{ker} \omega_{\hat{t}^{*}}
$$

Para a forma $\omega$ ser multissimplética e L ser o subespaço multilagrangeano pertinente, as seguintes condições são necessárias e suficientes:

- L é isotrópico;

- $\operatorname{dim} L=(n \operatorname{dim} V+1) /(n+1)$.

Para formular o teorema de Darboux (algébrico) no contexto específico de formas multissimpléticas, começamos por adaptar a Definição 1.6 a este contexto: explicitamente, uma base de Darboux multissimplética ou base canônica multissimplética é uma base

$$
\left\{e_{\mu}, e_{i}, e_{\nu}^{j}, e_{0} \mid 1 \leqslant \mu, \nu \leqslant n, 1 \leqslant i, j \leqslant N\right\}
$$

de $W$, com base dual

$$
\left\{e^{\mu}, e^{i}, e_{j}^{\nu}, e^{0} \mid 1 \leqslant \mu, \nu \leqslant n, 1 \leqslant i, j \leqslant N\right\}
$$

de $W^{*}$, tal que

- $\left\{e_{\mu} \mid 1 \leqslant \mu \leqslant n\right\}$ é uma base de um subespaço de $W$ complementar a $V$, isomorfo a $T$, e $\left\{e^{\mu} \mid 1 \leqslant \mu \leqslant n\right\}$ é a correspondente base dual do subespaço $V^{\perp}$ de $W^{*}$, de modo que se definirmos

$$
\hat{e}^{0}=e^{1} \wedge \ldots \wedge e^{n} \quad \text { e } \quad \hat{e}_{\mu}=\mathrm{i}_{e_{\mu}} \hat{e}^{0} \quad(1 \leqslant \mu \leqslant n)
$$

então $\hat{e}^{0}$ gera o espaço unidimensional $\bigwedge_{0}^{n} W^{*} \cong \bigwedge^{n} T^{*}$ e $\left\{\hat{e}_{\mu} \mid 1 \leqslant \mu \leqslant n\right\}$ é uma base do espaço $n$-dimensional $\bigwedge_{0}^{n-1} W^{*} \cong \bigwedge^{n-1} T^{*}$;

- $\left\{e_{i} \mid 1 \leqslant i \leqslant N\right\}$ é uma base de um subespaço de $V$ complementar a $L$;

- $\left\{e_{\mu}^{i}, e_{0} \mid 1 \leqslant \mu \leqslant n, 1 \leqslant i \leqslant N\right\}$ é uma base de $L$;

- $e_{0}$ gera o subespaço unidimensional ker $\hat{\omega}$; 
e tal que

$$
\omega=e^{i} \wedge e_{i}^{\mu} \wedge \hat{e}_{\mu}+e^{0} \wedge \hat{e}^{0} .
$$

e para o símbolo

$$
\hat{\omega}=\left(e^{i} \wedge e_{i}^{\mu}\right) \otimes \hat{e}_{\mu} .
$$

Obviamente, uma $(n+1)$-forma $\omega \in \Lambda_{2}^{n+1} W^{*}$ que admite uma base canônica deste tipo é uma forma multissimplética. Reciprocamente, como corolário do Teorema 1.11, temos

\section{Teorema 3.2 (Teorema de Darboux para espaços vetoriais multissimpléticos)}

Todo espaço multissimplético possui uma base canônica multissimplética.

Apesar deste teorema já ter sido provado no primeiro capítulo, queremos ainda apresentar uma demonstração alternativa baseada no teorema análogo para formas polissimpléticas (Teorema 3.1), usando o símbolo. Este procedimento usa fortemente o fato de que o núcleo do símbolo da forma multissimplética é apenas unidimensional, o que permite reduzir o problema ao caso polissimplético.

Demonstração. Começamos pela escolha de uma base qualquer $\left\{e_{\mu} \mid 1 \leqslant \mu \leqslant n\right\}$ de um subespaço de $W$ complementar a $V$, isomorfo a $T$, com base dual $\left\{e^{\mu} \mid 1 \leqslant \mu \leqslant n\right\}$ do subespaço $V^{\perp}$ em $W^{*}$, e definimos $\hat{e}^{0}=e^{1} \wedge \ldots \wedge e^{n}$ e $\hat{e}_{\mu}=\mathrm{i}_{e_{\mu}} \hat{e}^{0}$, como acima. Também definimos a 1-forma $e^{0}$ em $W$ por $e^{0}=(-1)^{n} \mathrm{i}_{e_{1}} \ldots \mathrm{i}_{e_{n}} \omega$, ou seja,

$$
\left\langle e^{0}, w\right\rangle=\omega\left(w, e_{1}, \ldots, e_{n}\right) \quad \text { para } w \in W .
$$

Como $\omega$ é não-degenerada, temos ker $e^{0} \cap \operatorname{ker} \hat{\omega}=\{0\}$. Assim, podemos escolher um vetor $e_{0} \in \operatorname{ker} \hat{\omega}$ tal que $\left\langle e^{0}, e_{0}\right\rangle=1$ e um subespaço $W_{0}$ qualquer de $W$ complementar a ker $\hat{\omega}$, pondo $V_{0}=V \cap W_{0}$ e $L_{0}=L \cap W_{0}$. Então a restrição do símbolo $\hat{\omega}$ de $\omega$ a $V_{0}$ é uma forma polissimplética não-degenerada a valores no espaço auxiliar $\hat{T}=\bigwedge_{0}^{n-1} W^{*} \cong \bigwedge^{n-1} T^{*}$, com subespaço polilagrangeano $L_{0}$, e portanto $V_{0}$ admite uma base canônica polissimplética $\left\{e_{i}, e_{\nu}^{j} \mid 1 \leqslant \nu \leqslant n, 1 \leqslant i, j \leqslant N\right\}$ para $\hat{\omega}$ adaptada à base $\left\{\hat{e}_{\mu} \mid 1 \leqslant \mu \leqslant n\right\}$ de $\hat{T}$. Agora, observando que a $(n+1)$-forma $(n-1)$-horizontal

$$
\omega-e^{0} \wedge \hat{e}^{0}
$$

é degenerada, com núcleo igual a ker $\hat{\omega}$, mas tem o mesmo símbolo $\hat{\omega}$ que a $(n+1)$-forma $(n-1)$-horizontal original $\omega$, concluímos que $\left\{e_{\mu}, e_{i}, e_{\nu}^{j}, e_{0} \mid 1 \leqslant \mu, \nu \leqslant n, 1 \leqslant i, j \leqslant N\right\}$ é uma base canônica multissimplética para $\omega$. 


\subsection{Variedades e fibrados poli- e multissimpléticos}

Os teoremas de Darboux do segundo capítulo garantem a existência de coordenadas locais canônicas nas quais uma forma polissimplética é expressa por

$$
\hat{\omega}=\left(d q^{i} \wedge d p_{i}^{a}\right) \otimes \hat{e}_{a}
$$

e uma forma multissimplética por

$$
\omega=d q^{i} \wedge d p_{i}^{\mu} \wedge d^{n} x_{\mu}-d p \wedge d^{n} x
$$

onde $d^{n} x_{\mu}=\mathrm{i}_{\partial_{\mu}} d^{n} x$, reproduzindo assim fielmente a estrutura canônica fundamental da teoria clássica dos campos.

Obviamente, o que foi feito neste trabalho não é o fim mas apenas o início. Esperamos que a teoria de variedades e fibrados poli- e multissimpléticos, para a qual acreditamos ter colocado o fundamento, possa em um futuro não muito distante se tornar tão interessante e frutífera quanto a geometria simplética. 

APÊNDICE A

\section{Lema de Poincaré}

Neste apêndice, apresentamos a versão do lema de Poincaré utilizada na demonstração do teorema de Darboux no final do Capítulo 2.

Teorema A.1 Seja $\omega \in \Omega^{k}(P, \hat{T})$ uma forma fechada em uma variedade $P$ tomando valores em um espaço vetorial fixo $\hat{T}$ e seja $L$ um subfibrado involutivo de TP. Suponha que $\omega$ é $(k-r)$-horizontal (relativo a $L)$, i.e., tal que para todo $p \in P$ e todo $v_{1}, \ldots, v_{r+1} \in L_{p}$, vale

$$
\mathrm{i}_{v_{1}} \ldots \mathrm{i}_{v_{r+1}} \omega_{p}=0 \text {. }
$$

Então em torno de cada ponto de $P$ existem uma vizinhança aberta $U$ deste ponto e uma $(k-1)$-forma $\theta \in \Omega^{k-1}(U, \hat{T})$ em $U$ que também é $(k-r)$-horizontal (relativo a L), i.e., tal que para todo $p \in U$ e todo $v_{1}, \ldots, v_{r} \in L_{p}$,

$$
\mathrm{i}_{v_{1}} \ldots \mathrm{i}_{v_{r}} \theta_{p}=0
$$

e tal que $\omega=d \theta$ em $U$.

DEMONSTRAÇÃo. Inicialmente, notamos que se trata de um teorema de caráter local, e como o subfibrado $L$ de TP é involutivo, podemos sem perda de generalidade trabalhar em uma carta local da variedade $P$ em torno do ponto de referência escolhido tal que a folheação correspondente é dada por um subespaço fixo do espaço modelo, i.e., através de um difeomorfismo, temos $P \cong K_{0} \oplus L_{0}$ com $L \cong L_{0}$ (a seguir, omitiremos o índice 0 ). Suporemos ainda que, nesta carta, o acima mencionado ponto de referência corresponde à origem 0 deste espaço vetorial. Finalmente, suporemos que $\hat{T}=\mathbb{R}$, pois caso contrário podemos provar a afirmação do teorema separadamente para cada componente de $\omega$ e $\theta$, relativo a alguma base de $\hat{T}$.

Para $t \in \mathbb{R}$, defina a " $K$-contração" $F_{t}^{K}: P \rightarrow P$ e a " $L$-contração" $F_{t}^{L}: P \rightarrow P$ por $F_{t}^{K}(x, y)=(t x, y)$ e $F_{t}^{L}(x, y)=(x, t y)$; obviamente, $F_{t}^{K}$ e $F_{t}^{L}$ são difeomorfismos se $t \neq 0$ 
e são projeções se $t=0$. Associado a cada uma destas famílias de aplicações existe um campo vetorial dependente do tempo que a gera no sentido de que, para $t \neq 0$,

$$
X_{t}^{K}\left(F_{t}^{K}(x, y)\right)=\left.\frac{d}{d s} F_{s}^{K}(x, y)\right|_{s=t} \quad \text { e } \quad X_{t}^{L}\left(F_{t}^{L}(x, y)\right)=\left.\frac{d}{d s} F_{s}^{L}(x, y)\right|_{s=t} .
$$

Explicitamente, para $t \neq 0$,

$$
X_{t}^{K}(x, y)=t^{-1}(x, 0) \quad \text { e } \quad X_{t}^{L}(x, y)=t^{-1}(0, y) .
$$

Defina $\omega_{0}=\left(F_{0}^{L}\right)^{*} \omega$ e, para $\epsilon>0$

$$
\theta_{\epsilon}=\int_{\epsilon}^{1} d t\left(\left(F_{t}^{L}\right)^{*}\left(\mathrm{i}_{X_{t}^{L}} \omega\right)+\left(F_{t}^{K}\right)^{*}\left(\mathrm{i}_{X_{t}^{K}} \omega_{0}\right)\right)
$$

assim como

$$
\theta=\lim _{\epsilon \rightarrow 0} \theta_{\epsilon}=\int_{0}^{1} d t\left(\left(F_{t}^{L}\right)^{*}\left(\mathrm{i}_{X_{t}^{L}} \omega\right)+\left(F_{t}^{K}\right)^{*}\left(\mathrm{i}_{X_{t}^{K}} \omega_{0}\right)\right) .
$$

Para ver que as $(k-1)$-formas $\theta$ e $\theta_{\epsilon}$ são bem definidas, considere $k-1$ vetores $\left(u_{i}, v_{i}\right) \in K \oplus L$ $(1 \leqslant i \leqslant k-1)$ e observe que, para $t \neq 0$,

$$
\left(F_{t}^{L}\right)^{*}\left(i_{X_{t}^{L}} \omega\right)_{(x, y)}\left(\left(u_{1}, v_{1}\right), \ldots,\left(u_{k-1}, v_{k-1}\right)\right)=\omega_{(x, t y)}\left((0, y),\left(u_{1}, t v_{1}\right), \ldots,\left(u_{k-1}, t v_{k-1}\right)\right)
$$

e

$$
\left(F_{t}^{K}\right)^{*}\left(i_{X_{t}^{K}} \omega_{0}\right)_{(x, y)}\left(\left(u_{1}, v_{1}\right), \ldots,\left(u_{k-1}, v_{k-1}\right)\right)=t^{k-1} \omega_{(t x, 0)}\left((x, 0),\left(u_{1}, 0\right), \ldots,\left(u_{k-1}, 0\right)\right) .
$$

Aqui nós vemos facilmente que ambas as expressões são diferenciáveis em $t$ e constituem, respectivamente, uma $(k-1)$-forma $(k-r)$-horizontal e uma $(k-1)$-forma $(k-1)$-horizontal relativo a $L$. Assim, $\theta_{\epsilon}$ e $\theta$ também são $(k-1)$-formas $(k-r)$-horizontais relativo a $L$. Além disso, como $d \omega=0$ e $d \omega_{0}=0$,

$$
\begin{aligned}
d \theta_{\epsilon} & =\int_{\epsilon}^{1} d t\left(\left(F_{t}^{L}\right)^{*}\left(\mathrm{i}_{X_{t}^{L}} \omega\right)+\left(F_{t}^{K}\right)^{*}\left(\mathrm{i}_{X_{t}^{K}} \omega_{0}\right)\right)=\int_{\epsilon}^{1} d t\left(\left(F_{t}^{L}\right)^{*}\left(L_{X_{t}^{L}} \omega\right)+\left(F_{t}^{K}\right)^{*}\left(L_{X_{t}^{K}} \omega_{0}\right)\right) \\
& =\int_{\epsilon}^{1} d t\left(\frac{d}{d t}\left(\left(F_{t}^{L}\right)^{*} \omega\right)+\frac{d}{d t}\left(\left(F_{t}^{K}\right)^{*} \omega_{0}\right)\right)=\omega-\left(F_{\epsilon}^{L}\right)^{*} \omega+\omega_{0}-\left(F_{\epsilon}^{K}\right)^{*} \omega_{0} .
\end{aligned}
$$

Tomando o limite $\epsilon \rightarrow 0$, temos $\left(F_{\epsilon}^{L}\right)^{*} \omega \rightarrow \omega_{0}$ e $\left(F_{\epsilon}^{K}\right)^{*} \omega_{0} \rightarrow 0$ e assim, $d \theta=\omega$. 


\section{Bibliografia}

[1] R. Abraham \& J.E. Marsden: Foundations of Mechanics, $2^{\text {nd }}$ edition, BenjaminCummings, Reading 1978.

[2] A. Awane: k-symplectic structures, J. Math. Phys. 33 (1992) 4046-4052.

[3] F. Cantrijn, A. Ibort \& M. De León: On the Geometry of Multisympletic Manifolds, J. Austral. Math. Soc. (Series A) 66 (1999) 303-330.

[4] J.F. Cariñena, M. Crampin \& L.A. Ibort: On the Multisymplectic Formalism for First Order Field Theories, Diff. Geom. App. 1 (1991) 345-374.

[5] Th. De Donder: Théorie Invariante du Calcul des Variations, Gauthier-Villars, Paris 1935.

[6] M. Forger, C. Paufler \& H. Römer: The Poisson Bracket for Poisson Forms in Multisymplectic Field Theory, Rev. Math. Phys. 15 (2003) 705-743.

[7] M. Forger, C. Paufler \& H. Römer: Hamiltonian Multivector Fields and Poisson Forms in Multisymplectic Field Theory, J. Math. Phys. 46 (2005) 112903, 29 pp.

[8] M. Forger \& S. Romero: Covariant Poisson Brackets in Geometric Field Theory, Commun. Math. Phys. 256 (2005) 375-410.

[9] M. Gotay, J. Isenberg, J. Marsden, R. Montgomery Momentum Maps and Classical Fields. Part I: Covariant Field Theory, arXiv: physics/9801019v2.

[10] W.H. Greub, S. Halperin \& R. Vanstone: Connections, Curvature and Cohomology, Academic Press, New York (Vol. 1, 1972; Vol. 2, 1973) 
[11] M. Grassi: Polysymplectic Spaces, s-Kähler Manifolds and Lagrangian Fibrations, arXiv: math.DG/0006154v1.

[12] C. Günther: The Polysymplectic Hamiltonian Formalism in Field Theory and Calculus of Variations I: the Local Case, J. Diff. Geom. 25 (1987) 23-53.

[13] J. Kijowski: A Finite-dimensional Canonical Formalism in the Classical Field Theory, Commun. Math. Phys. 30 (1973) 99-128; Multiphase Spaces and Gauge in Calculus of Variations, Bull. Acad. Pol. Sci. SMAP 22 (1974) 1219-1225.

[14] J. Kijowski \& W. Szczyrba: Multisymplectic Manifolds and the Geometrical Construction of the Poisson Brackets in the Classical Field Theory, in: "Géometrie Symplectique et Physique Mathématique", pp. 347-379, ed.: J.-M. Souriau, C.N.R.S., Paris 1975.

[15] J. Kijowski \& W. Szczyrba: Canonical Structure for Classical Field Theories, Commun. Math. Phys. 46 (1976) 183-206.

[16] S. Kobayashi \& K. Nomizu: Foundations of Differential Geometry, Interscience, New York (Vol. 1, 1963; Vol. 2, 1969)

[17] M. De León, D. de Diego e Santamaría-Merino: Tulczyjew's Triples and Lagrangian Submanifolds in Classical Field Theories, in: W. Sarlet \& F. Cantrjin (eds): Applied Differential Geometry and Mechanics, Univ. of Gent, Gent, Academic Press (2003) 2147; arXiv: math-ph/03020026.

[18] J. Martinet: Sur les Singularités des Formes Différentielles, Ann. Inst. Four. 20 (1970) 95-178.

[19] G. Martin: A Darboux Theorem for Multisymplectic Manifolds, Lett. Math. Phys. 16 (1988) 133-138.

[20] H. Weyl: Geodesic Fields in the Calculus of Variations for Multiple Integrals, Ann. Math. 36 (1935) 607-629. 